J. DIFFERENTIAL GEOMETRY

81 (2009) 91-142

\title{
TRIANGULATED CORES OF PUNCTURED-TORUS GROUPS
}

\author{
FrançOIS GuÉritaud
}

\begin{abstract}
We show that the interior of the convex core of a quasifuchsian punctured-torus group admits an ideal decomposition (usually an infinite triangulation) which is canonical in two very different senses: in a combinatorial sense via the pleating invariants, and in a geometric sense via an Epstein-Penner convex hull construction in Minkowski space. This result re-proves the Pleating Lamination Theorem for quasifuchsian punctured-torus groups, and extends to all punctured-torus groups if a strong version of the Pleating Lamination Conjecture is true.
\end{abstract}

\section{Introduction}

Among Kleinian groups with infinite covolume, quasifuchsian groups, which are deformations of Fuchsian surface groups, are fundamental examples. It has been noted $[\mathbf{J} \varnothing, \mathbf{M S W}]$ that such groups can be analyzed much more explicitly when the base surface is just a once-punctured torus - the curve complex is then dual to a planar tree, which simplifies many issues. Punctured-torus groups (which still retain many features of general quasifuchsian groups) have thus become a favorite "training ground": Minsky's work [Min] on end invariants is an example. Similarly, Caroline Series [Se] was able to prove the Pleating Lamination Conjecture for quasifuchsian punctured-torus groups (general pleating laminations, in contrast, seem to pose tremendous technical challenges: see $[\mathbf{B O}]$ for the best known results). In this paper, we prove that the interior of the convex core of any quasifuchsian punctured-torus group admits an ideal triangulation (or slightly more general decomposition) which relates "as nicely as one could possibly hope" both to the intrinsic geometry of the convex core, and to the combinatorics of its boundary pleatings. This answers several conjectures made in [ASWY2]. As a byproduct, we get enough control to re-prove the aforementioned result of $[\mathbf{S e}]$.

Let $M=\mathbb{H}^{3} / \Gamma$ be a hyperbolic manifold with one cusp. Akiyoshi and Sakuma $[\mathbf{A S}]$ generalized the Epstein-Penner convex hull construction

Received 05/29/2006. 
in Minkowski space $\mathbb{R}^{3+1}$ (see $[\mathbf{E P}]$ ) to show that the interior $V$ of the convex core of $M$ admits a (unique) decomposition $\mathcal{D}^{\text {Geom }}$, canonical in a purely geometric sense. Roughly speaking, $\mathcal{D}^{\text {Geom }}$ is defined by considering the convex hull $C \subset \mathbb{R}^{3+1}$ of the discrete $\Gamma$-orbit of any light-like (i.e., isotropic) vector representing the cusp, and by projecting the cell decomposition of $\partial C$ back to $M$ (see Section 9 for more detail).

This decomposition $\mathcal{D}^{\text {Geom }}$ of $V$ is quite mysterious in general. It seems to be unknown, for instance, whether $\mathcal{D}^{\text {Geom }}$ is always locally finite (see $[\mathbf{A S}]$ ). However, those top-dimensional cells of $\mathcal{D}^{\text {Geom }}$ which come from space-like faces of $C$ are contractible ideal polyhedra, and are dual to the vertices of the Ford-Voronoi domain of $M$. Other topdimensional cells of $\mathcal{D}^{\text {Geom }}$ may be non-contractible, are not seen in the Ford-Voronoi domain, and will typically accumulate near $\partial V$.

For quasifuchsian punctured-torus groups (i.e., geometrically finite discrete subgroups of $\operatorname{Isom}^{+}\left(\mathbb{H}^{3}\right)$ freely generated by two elements with parabolic commutator), a nice guess can be made at the combinatorics of $\mathcal{D}^{\text {Geom }}$. Indeed, the complex of ideal triangulations of the punctured torus is a tree $T$, and the projective classes of the two boundary pleatings of $V$ can be seen as two points at infinity in $T$ : the unique geodesic in $T$ connecting these two points will define an explicit combinatorial ideal triangulation $\mathcal{D}^{\text {Comb }}$ of $V$ (see Section 1 for detail). The triangulation $\mathcal{D}^{\mathrm{Comb}}$ is canonical in a combinatorial sense with respect to the pleating data of $V$. It is natural to ask if $\mathcal{D}^{\text {Comb }}$ can be straightened out to be made a totally geodesic triangulation of $V$ (without turning any tetrahedra "inside out"). Our main theorem answers this question in the affirmative:

Theorem 0.1. If $V$ is the interior of the convex core of a quasifuchsian punctured-torus group, the ideal triangulation $\mathcal{D}^{\text {Geom }}$ of $V$ is the totally geodesic straightening of $\mathcal{D}^{\mathrm{Comb}}$.

In fact, aside from $\mathcal{D}^{\text {Comb }}$ and its coarsenings, the author does not know of any ideal cell decomposition that is invariant under the hyperelliptic involution (a property which $\mathcal{D}^{\text {Geom }}$ must enjoy by symmetry).

Thurston and Bonahon [B1] showed how to associate a projective class of measured laminations, or ending lamination, to a geometrically infinite end of a hyperbolic manifold. The Ending Lamination Theorem $[\mathbf{B C M}]$ roughly states that the asymptotic geometry of the end is completely described by its ending lamination. In view of extending Theorem 0.1 to all punctured-torus groups, it is reasonable to understand ending laminations as infinitely strong pleatings (see Section 10-D), and to conjecture that the group is determined by its ending and/or pleating laminations (this is a strong version of the Pleating Lamination Conjecture). Indeed, our method allows to construct punctured-torus groups with arbitrary admissible ending and/or pleating laminations, and these groups satisfy an analogue of Theorem 0.1: the precise statement with a 
full description of $\mathcal{D}^{\text {Geom }}$ (especially in the case of rational laminations) is Theorem 10.1 in Section 10. When exactly one of the two ends is geometrically infinite, the uniqueness of these groups remains an open problem.

The identity $\mathcal{D}^{\text {Geom }}=\mathcal{D}^{\text {Comb }}=: \mathcal{D}$ of Theorem 0.1 (and the existence of $\mathcal{D}^{\mathrm{Comb}}$, as realized by positively oriented cells) was Conjecture $8.2 \mathrm{in}$ [ASWY2], also called the Elliptic-Parabolic-Hyperbolic (EPH) Conjecture. Akiyoshi subsequently established the identity in the case of two infinite ends in $[\mathbf{A k}]$. Near a finite end, however, the ideal tetrahedra of $\mathcal{D}$ flatten at a very quick rate: the smallest angle of the $i$-th tetrahedron typically goes to 0 faster than any geometric sequence $\left(\alpha^{i}\right)_{i \geq 0}$. In a sense, the difficulty is to show that these angles nevertheless stay positive for the hyperbolic metric.

The geometric realization of $\mathcal{D}^{\text {Comb }}$ (i.e., the construction of angles for the tetrahedra) will be fairly explicit: the solution will arise as the maximum of an explicit concave "volume" functional $\mathcal{V}$ over an explicit convex domain. The domain has infinite dimension, but there are explicit bounds on the contributions to $\mathcal{V}$ of the "tail" coordinates (suggesting efficient numerical implementations). This feature is also what allows us to show that the Kleinian group is a continuous function of its pleatings, which is the key to the Pleating Lamination Theorem (Section 10-E).

Acknowledgements. My thanks go to Makoto Sakuma for having drawn my attention to the EPH conjecture. I am also deeply indebted to Francis Bonahon, Frédéric Paulin, Makoto Sakuma, and David Futer for many discussions, insights, and suggestions.

\section{Strategy}

The triangulation $\mathcal{D}^{\text {Geom }}$ can be seen (dually) as a refinement of the Ford-Voronoi domain of the quasifuchsian punctured-torus group. The latter domain was described in great detail in $[\mathbf{J} \boldsymbol{\varnothing}, \mathbf{A S W Y 1 , ~ A S W Y 2 ] ~}$ and [ASWY3]: this body of work produced a combinatorial description of finitely many tetrahedra of $\mathcal{D}^{\text {Geom }}$, based on a deformational or "geometric continuity" argument in the (connected) space of all quasifuchsian groups. To study all tetrahedra at once, including those not seen in the Ford-Voronoi domain, the present paper takes a somewhat opposite approach: first describe the candidate triangulation $\mathcal{D}^{\mathrm{Comb}}$ and find geometric shapes for its tetrahedra, then establish that the gluing of these tetrahedra defines (the interior of the convex core of) a quasifuchsian group. A computation in Minkowski space eventually yields Theorem $0.1\left(\mathcal{D}^{\mathrm{Comb}}=\mathcal{D}^{\mathrm{Geom}}\right)$, as a bonus, so to speak.

Identities of the form $\mathcal{D}^{\text {Comb }}=\mathcal{D}^{\text {Geom }}$ have been established by specialized techniques in several related contexts: punctured-torus bundles 
(by Lackenby [La]); complements of two-bridge links (see the announcement in [ASWY3]) or of certain arborescent links (see [G2]); totally degenerate punctured-torus groups $[\mathbf{A k}]$. The methods of the present paper essentially cover all these cases, but its true novelty is in dealing with pleating laminations, i.e., with groups whose limit set is smaller than $\mathbb{P}^{1} \mathbb{C}$. We will build on results (and notation) from $[\mathbf{G F}]$.

1-A. Setup. Let $S:=\left(\mathbb{R}^{2} \backslash \mathbb{Z}^{2}\right) / \mathbb{Z}^{2}$ be the once-punctured torus endowed with its differential structure. Let $\mathcal{C}$ be the set of isotopy classes of simple closed curves in $S$ that are not the loop around the puncture. Let also $\mathcal{C}^{\prime}$ be the set of isotopy classes of properly embedded lines (running from the puncture to itself) in $S$ that do not bound a disk.

Consider the maximal abelian cover $\pi: \mathbb{R}^{2} \backslash \mathbb{Z}^{2} \rightarrow S$ and two coprime integers $\xi, \eta$. If $\ell$ is a line of slope $\eta / \xi$ in $\mathbb{R}^{2}$ missing (resp. intersecting) $\mathbb{Z}^{2}$, then $\pi\left(\ell \backslash \mathbb{Z}^{2}\right)$ defines a class $\gamma \in \mathcal{C}$ (resp. $\left.\gamma^{\prime} \in \mathcal{C}^{\prime}\right)$. We call $\eta / \xi \in \mathbb{P}^{1} \mathbb{Q}$ the slope of $\gamma\left(\right.$ resp. $\left.\gamma^{\prime}\right)$. It is well-known that both $\mathcal{C}$ and $\mathcal{C}^{\prime}$ are parametrized by $\mathbb{P}^{1} \mathbb{Q}=\mathbb{Q} \cup\{\infty\}$ via the slope: we have bijections

$$
s: \mathcal{C} \simeq \mathbb{P}^{1} \mathbb{Q} ; \quad s^{\prime}: \mathcal{C}^{\prime} \simeq \mathbb{P}^{1} \mathbb{Q} .
$$

When there is no ambiguity, we will sometimes omit $s$ and simply identify $\mathcal{C}$ with $\mathbb{P}^{1} \mathbb{Q}$.

We will focus primarily on irrational pleating laminations (for rational ones, see Section 10). Let $\left(\alpha^{+}, \beta^{+}\right)$and $\left(\alpha^{-}, \beta^{-}\right)$be elements of $\mathbb{R}^{* 2}$ such that $\beta^{+} / \alpha^{+}$and $\beta^{-} / \alpha^{-}$are distinct irrationals. Define the pleatings $\lambda^{ \pm}: \mathbb{P}^{1} \mathbb{Q} \rightarrow \mathbb{R}^{ \pm}$by

$$
\lambda^{+}(\eta / \xi)=\left\|\begin{array}{cc}
\beta^{+} & \eta \\
\alpha^{+} & \xi
\end{array}\right\| \text { and } \lambda^{-}(\eta / \xi)=-\left\|\begin{array}{cc}
\beta^{-} & \eta \\
\alpha^{-} & \xi
\end{array}\right\|,
$$

where the double bars denote the absolute value of the determinant (and $\xi, \eta$ are coprime integers). Replacing a pair $\left(\alpha^{ \pm}, \beta^{ \pm}\right)$by its negative does not change $\lambda^{ \pm}$. Notice that $\lambda^{-}<0<\lambda^{+}$(as functions on $\mathbb{P}^{1} \mathbb{Q}$ ).

In the hyperbolic plane $\mathbb{H}^{2}$ with boundary $\partial \mathbb{H}^{2}=\mathbb{P}^{1} \mathbb{R} \supset \mathbb{P}^{1} \mathbb{Q}$, consider the Farey triangulation (the ideal triangle $01 \infty$ iteratedly reflected in its sides, see e.g. Section 2 of [Min]). The irrationals $\beta^{+} / \alpha^{+}$and $\beta^{-} / \alpha^{-}$belong to $\partial \mathbb{H}^{2}$, and the oriented (geodesic) line $\Lambda$ from $\beta^{-} / \alpha^{-}$ to $\beta^{+} / \alpha^{+}$crosses infinitely many Farey edges $\left(e_{i}\right)_{i \in \mathbb{Z}}$ (the choice of $e_{0}$ is arbitrary). To every pair of consecutive integers $(i-1, i)$ is associated a letter, $R$ or $L$, according to whether $e_{i-1}$ and $e_{i}$ share their Right or Left end with respect to the orientation of $\Lambda$ (we say that $\Lambda$ makes a Right or makes a Left across the Farey triangle between $e_{i-1}$ and $e_{i}$ ). We thus get a bi-infinite word ...RLLLRR ... with infinitely many $R$ 's (resp. L's) near either end.

Two distinct rationals of $\mathbb{P}^{1} \mathbb{Q}$ are Farey neighbors exactly when the corresponding elements (properly embedded lines) of $\mathcal{C}^{\prime}$ are disjoint up 
to homotopy. Therefore, each Farey triangle $\tau$ defines an ideal triangulation of the punctured torus $S$ : indeed, the vertices of $\tau$ are the slopes of three disjoint (properly embedded) lines of $\mathcal{C}^{\prime}$, whose union separates $S$ into two ideal triangles. Moreover, two triangulations of $S$ corresponding to Farey triangles which share a Farey edge always differ by a diagonal move (see Figure 1). Such a diagonal move must be seen as a (topological) ideal tetrahedron in $S \times \mathbb{R}$ filling the space between two (topological) pleated surfaces, pleated along the two ideal triangulations.
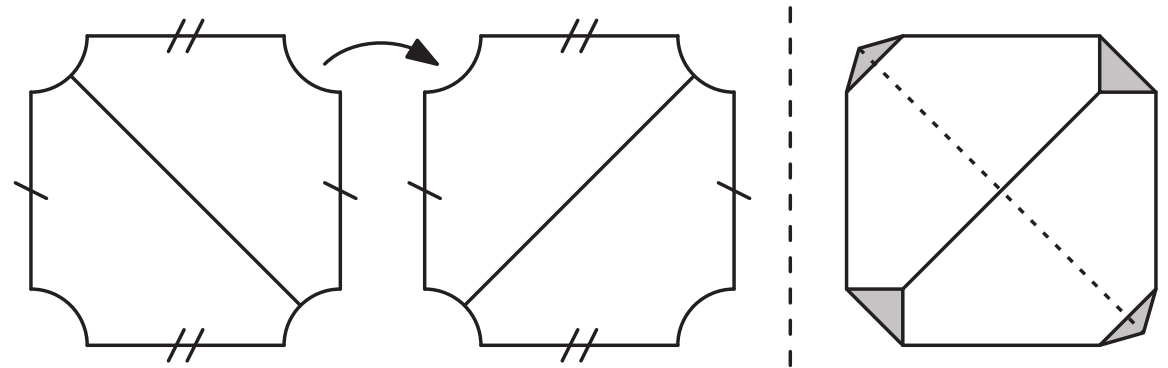

Figure 1. Left: a diagonal move in $S$ (the puncture is in the corners), associated to adjacent Farey triangles $-1 \infty 0$ and $1 \infty 0$. Right: how to see the diagonal move as an ideal tetrahedron, here with truncated vertices (grey).

Our strategy will be to consider triangulated surfaces $S_{i}$ (pleated punctured tori) corresponding to the Farey triangles living between $e_{i-1}$ and $e_{i}$ for $i$ ranging over $\mathbb{Z}$, interpolate these surfaces with tetrahedra $\Delta_{i}$ corresponding to the diagonal moves (i.e., to the Farey edges $e_{i}$ themselves), and provide geometric parameters (dihedral angles) for all these objects, using Rivin's Maximum Volume Principle (see [Ri]). Note that the irrationals $\frac{\beta^{-}}{\alpha^{-}}, \frac{\beta^{+}}{\alpha^{+}}$encode all of the combinatorial gluing rule between $\Delta_{i-1}$ and $\Delta_{i}$. Also, each $S_{i} \subset S \times \mathbb{R}$, being homotopic to a fiber $S \times\{*\}$, comes with a natural marking $S_{i} \simeq S$ (defined up to isotopy).

Theorem 1.1. There exists a cusped, non-complete hyperbolic 3manifold $V$ homeomorphic to $S \times \mathbb{R}$ such that:

(i) The metric completion $\bar{V}$ of $V$ is homeomorphic to $S \times[0,1]=V \sqcup$ $S_{-\infty} \sqcup S_{+\infty}$, where $S_{-\infty}, S_{+\infty}$ are locally convex pleated surfaces whose pleating measures restrict to $\lambda^{-} \circ s$ and $\lambda^{+} \circ s$ (respectively) on the set $\mathcal{C}$ of simple closed curves, where $s$ is the slope function.

(ii) The geometric realizations of the $\left\{\Delta_{i}\right\}_{i \in \mathbb{Z}}$ in $V$ define a totally geodesic ideal triangulation $\mathcal{D}^{\text {Comb }}$ of $V$.

Sections 2 to 8 are devoted to producing such a $V$. The puncture of $S$ is required to correspond to a cusp of $V$, so $\bar{V}$ (being convex) will be the 
convex core of a hyperbolic manifold $\mathbb{H}^{3} / \Gamma$, where $\Gamma$ is a quasifuchsian punctured-torus group (and we say that $\bar{V}$ has pleating data $\lambda^{ \pm}$).

By [Se], or Corollary 10.8 below, the group $\Gamma$ is determined uniquely up to conjugacy in $\operatorname{Isom}\left(\mathbb{H}^{3}\right)$ by the $\lambda^{ \pm}$. Our construction therefore provides a decomposition $\mathcal{D}^{\text {Comb }}$ of the interior $V$ of the convex core of an arbitrary quasifuchsian punctured-torus group $\Gamma$ with irrational pleating laminations. The universal cover of $V$ is tentatively drawn in Figure 3 of $[\mathbf{T h}]$ (though the faces ought to be infinite polygons, not triangles).

Throughout the paper, we will deal with an infinite family of tetrahedra $\left(\Delta_{i}\right)_{i \in \mathbb{Z}}$, separated by pleated once-punctured tori $\left(S_{i}\right)_{i \in \mathbb{Z}}$. By an arbitrary choice, we have resolved that $S_{i}$ is the surface between $\Delta_{i}$ and $\Delta_{i-1}$ - or equivalently, that $\Delta_{i}$ is bounded by the surfaces $S_{i}$ and $S_{i+1}$. However, the numbering of the tetrahedra should be seen as the more essential one (see especially Definition 3.1 below).

1-B. Plan of the paper. In Section 2 (borrowed from $[\mathbf{G F}]$ ), we describe the space of possible dihedral angle assignments $x_{i}, y_{i}, z_{i}$ for the $\left\{\Delta_{i}\right\}_{i \in \mathbb{Z}}$. In Section 3, we encode $\lambda^{ \pm}$into constraints on the $x_{i}, y_{i}, z_{i}$. In Section 4, we carry out (constrained) volume maximization. Important asymptotic features of the solution are analyzed in Section 5. In Section 6, we describe the Euclidean triangulation of the cusp. In Sections 7 and 8, we show that the pleated surfaces $S_{i}$ converge in a strong enough sense, so that their limit as $i$ goes to $\pm \infty$ describes the (pleated) boundary of the metric completion of $V=\bigcup_{i \in \mathbb{Z}} \Delta_{i}$. At that point, we have constructed (the convex core of) a quasifuchsian group. The corresponding instance of Theorem 0.1 then follows from a computation, carried out in Section 9. In Section 10, we provide a similar construction of punctured-torus groups with rational pleating slopes $\beta^{ \pm} / \alpha^{ \pm}$and/or with infinite ends, and re-prove that $\left(\lambda^{+}, \lambda^{-}\right)$are continuous coordinates for the space of quasifuchsian groups (see $[\mathbf{S e}]$ ).

\section{Dihedral angles}

In this section, we find positive dihedral angles for the ideal tetrahedra $\Delta_{i}$, following Section 5 of $[\mathbf{G F}]$. More precisely, we describe the convex space $\Sigma$ of positive angle configurations for the $\Delta_{i}$ such that:

- the three dihedral angles near each ideal vertex of $\Delta_{i}$ add up to $\pi$ (this is true in any ideal tetrahedron of $\mathbb{H}^{3}$ );

- the dihedral angles around any edge of $V=\bigcup_{i \in \mathbb{Z}} \Delta_{i}$ add up to $2 \pi$ (this is necessary, though not sufficient, for a hyperbolic structure at the edge);

- the three pleating angles of each pleated punctured torus $S_{i}$ add up to 0 (this is necessary, though not sufficient, to make the puncture $p$ of $S$ a cusp of $V$, i.e., make the loop around $p$ lift to a parabolic isometry of $\mathbb{H}^{3}$ ). 
(The first condition implies that opposite edges in $\Delta_{i}$ have the same dihedral angle.) Later on, we shall apply Rivin's Maximum Volume Principle [Ri] on a certain convex subset of (the closure of) $\Sigma$.

If the tetrahedron $\Delta_{i}$ realizes a diagonal move (Figure 1) that kills an edge $\varepsilon^{\prime}$ of $S_{i}$ and replaces it with $\varepsilon$, denote by $\pi-w_{i}$ the interior dihedral angle of $\Delta_{i}$ at $\varepsilon$ and $\varepsilon^{\prime}$. Observe that the slope of $\varepsilon$ (resp. $\varepsilon^{\prime}$ ) is the rational located opposite the Farey edge $e_{i}$ in the Farey diagram, on the side of $\beta^{+} / \alpha^{+}\left(\operatorname{resp} \beta^{-} / \alpha^{-}\right)$.

Thus, the pleating angles of the surface $S_{i}$ living between $\Delta_{i-1}$ and $\Delta_{i}$ are

$$
w_{i-1}, \quad-w_{i} \quad \text { and } \quad w_{i}-w_{i-1} \text {. }
$$

Observe the sign convention: the pleated punctured torus embedded in $S \times \mathbb{R}$ receives an upward transverse orientation from $\mathbb{R}$, and the angles we consider are the dihedral angles above the surface, minus $\pi$. Thus, the "new" edge of $\Delta_{i-1}$, pointing upward, accounts for a positive pleating $w_{i-1}$, while the "old" edge of $\Delta_{i}$, pointing downward, accounts for a negative pleating $-w_{i}$. This is in accordance with the convention $\lambda^{-}<0<\lambda^{+}$of (1), as we expect $V=\bigcup_{i \in \mathbb{Z}} \Delta_{i}$ to be convex. We may write the three numbers (2) in the associated corners of the Farey triangle corresponding to $S_{i}$ (Figure 2, top) and repeat the operation for $S_{i+1}$ (bottom). For notational convenience, we write $\left(w_{i-1}, w_{i}, w_{i+1}\right)=(a, b, c)$.
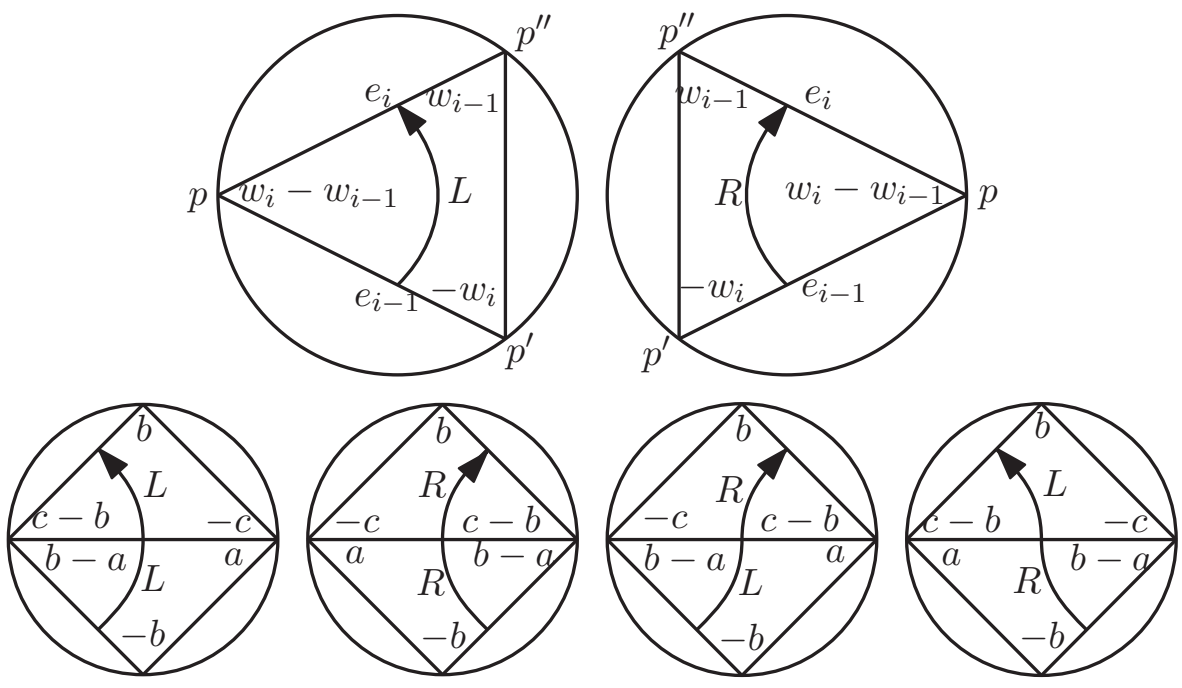

Figure 2. Bottom: $e_{i}$ is the horizontal edge and $(a, b, c)$ $=\left(w_{i-1}, w_{i}, w_{i+1}\right)$.

In the tetrahedron $\Delta_{i}$, let $x_{i}$ (resp. $y_{i}$ ) be the interior dihedral angle at the edge whose slope is given by the right (resp. left) end of the Farey 
F. GUÉRITAUD

edge $e_{i}$. Let $z_{i}=\pi-w_{i}$ be the third dihedral angle of $\Delta_{i}$. For instance, $2 x_{i}$ (resp. $2 y_{i}$ ) is the difference between the numbers written just below and just above the right (resp. left) end of $e_{i}$ in Figure 2 (the factor 2 comes from the fact that the two edges of $\Delta_{i}$ with angle $x_{i}$ [resp. $y_{i}$ ] are identified). By computing differences between the pleating angles given in Figure 2 (bottom), we find the following formulae for $x_{i}, y_{i}, z_{i}$ (depending on the letters, $R$ or $L$, living just before and just after the Farey edge $\left.e_{i}\right)$ :

\begin{tabular}{c|c|c|c|c} 
& $L L$ & $R R$ & $L R$ & $R L$ \\
\hline$x_{i}$ & $\frac{1}{2}(a+c)$ & $\frac{1}{2}(-a+2 b-c)$ & $\frac{1}{2}(a+b-c)$ & $\frac{1}{2}(-a+b+c)$ \\
$y_{i}$ & $\frac{1}{2}(-a+2 b-c)$ & $\frac{1}{2}(a+c)$ & $\frac{1}{2}(-a+b+c)$ & $\frac{1}{2}(a+b-c)$ \\
$z_{i}$ & $\pi-b$ & $\pi-b$ & $\pi-b$ & $\pi-b$
\end{tabular}

The first of the three conditions defining $\Sigma$ can be checked immediately; the other two are true by construction. From (3), the condition for all angles to be positive is that:

$\left\{\begin{array}{l}\text { For all } i \in \mathbb{Z} \text { one has } 0<w_{i}<\pi \\ \text { If } i \text { separates identical letters (first two cases), } 2 w_{i}>w_{i+1}+w_{i-1} \\ \text { If } i \text { separates different letters (last two cases), }\left|w_{i+1}-w_{i-1}\right|<w_{i}\end{array}\right.$

Denote by $\Sigma$ the non-empty, convex solution set of (4).

\section{Bounding the Bending}

3-A. A natural constraint on the pleating of $S_{i}$. Next, we describe a certain convex subset of $\Sigma$. It will be defined by decay conditions on the $\left\{w_{i}\right\}_{i \in \mathbb{Z}}$ near $\pm \infty$. These conditions will encode the pleating functions $\lambda^{ \pm}$, and are obtained in the following way. Consider the pleated punctured torus, $S_{i}$, lying between the tetrahedra $\Delta_{i}$ and $\Delta_{i-1}$. Let $\epsilon_{1}, \epsilon_{2}, \epsilon_{3}$ be the edges of $S_{i}$ and $\delta_{1}, \delta_{2}, \delta_{3}$ the corresponding pleating angles (exterior dihedral angles, counted positively for salient edges as in (2) above). Recall the set $\mathcal{C}$ of (isotopy classes of) simple closed curves in the punctured torus $S$. Define the pleating measure $\lambda_{i}: \mathcal{C} \rightarrow \mathbb{R}^{+}$of $S_{i}$ by

$$
\lambda_{i}(\gamma)=\delta_{1} \nu_{1}(\gamma)+\delta_{2} \nu_{2}(\gamma)+\delta_{3} \nu_{3}(\gamma),
$$

where $\nu_{\sigma}(\gamma) \in \mathbb{N}$ is the geometric intersection number of $\gamma$ with the edge $\epsilon_{\sigma}$. Denote by $\varepsilon_{\sigma} \in \mathcal{C}$ the simple closed curve that has the same slope as $\epsilon_{\sigma}$. We shall adopt the constraint that

$$
\lambda^{-}\left(\varepsilon_{\sigma}\right)<\lambda_{i}\left(\varepsilon_{\sigma}\right)<\lambda^{+}\left(\varepsilon_{\sigma}\right)
$$


for each $\sigma \in\{1,2,3\}$, and the same thing for every pleated punctured torus $S_{i}$ in the $\mathbb{Z}$-family. (Note: in fact, a similar inequality will follow for all simple closed curves $\gamma$ of $\mathcal{C}$ [see Lemma 3.5 below], namely, $\lambda^{-}<$ $\lambda_{i}<\lambda^{+}$as functions on $\mathcal{C}$. Forcing this "natural" inequality is the whole point of our constraint.)

In other words, denote by $\eta / \xi$ the (rational) slope of $\varepsilon_{\sigma}$. Observe that $\lambda_{i}\left(\varepsilon_{\sigma}\right)=\delta_{\sigma^{\prime}}+\delta_{\sigma^{\prime \prime}}=-\delta_{\sigma}$ where $\left\{\sigma, \sigma^{\prime}, \sigma^{\prime \prime}\right\}=\{1,2,3\}$, because the slopes of $\varepsilon_{1}, \varepsilon_{2}, \varepsilon_{3}$ are Farey neighbors. Therefore, by (1), the requirement is that for every $\varepsilon_{\sigma}$ as above,

$$
-\left\|\begin{array}{cc}
\beta^{+} & \eta \\
\alpha^{+} & \xi
\end{array}\right\|<\delta_{\sigma}<\left\|\begin{array}{cc}
\beta^{-} & \eta \\
\alpha^{-} & \xi
\end{array}\right\|
$$

To express (6) in terms of the $w_{i}$, we need some notation (the $\delta_{\sigma}$ are determined by the $w_{i}$ via (2) above). For each Farey edge $e_{i}$, let $q_{i}^{+}$ (resp. $q_{i}^{-}$) be the rational located opposite $e_{i}$, on the same side of $e_{i}$ as $\beta^{+} / \alpha^{+}$(resp. $\beta^{-} / \alpha^{-}$). For an arbitrary rational $p=\eta / \xi$ (reduced form), introduce the slightly abusive notation (abusive in that it depends on the ordered pair $(\alpha, \beta)$ rather than just on the real $\left.\frac{\beta}{\alpha}\right)$ :

$$
\frac{\beta}{\alpha} \wedge p:=\left\|\begin{array}{cc}
\beta & \eta \\
\alpha & \xi
\end{array}\right\| \text { (absolute value of the determinant). }
$$

Then, if $l, r$ are the rationals living at the left and right ends of the Farey edge $e_{i}$, one has

$$
\left(\frac{\beta^{+}}{\alpha^{+}} \wedge l\right)+\left(\frac{\beta^{+}}{\alpha^{+}} \wedge r\right)=\frac{\beta^{+}}{\alpha^{+}} \wedge q_{i}^{-} ;\left(\frac{\beta^{-}}{\alpha^{-}} \wedge l\right)+\left(\frac{\beta^{-}}{\alpha^{-}} \wedge r\right)=\frac{\beta^{-}}{\alpha^{-}} \wedge q_{i}^{+} .
$$

Indeed, the $\|\cdot\|$-notation is invariant under $P S L_{2}(\mathbb{Z})$, acting on $\left(\mathbb{H}^{2}, \partial \mathbb{H}^{2}\right)$ by isometries and on ordered pairs $\pm(\alpha, \beta)$ as a matrix group. Since $P S L_{2}(\mathbb{Z})$ acts transitively on oriented Farey edges, we are reduced to the case $\left(l, r, q_{i}^{+}, q_{i}^{-}\right)=(\infty, 0,1,-1)$ where $\beta^{-} / \alpha^{-}<0<\beta^{+} / \alpha^{+}$, which is straightforward.

Let us now translate Equation (6) in terms of the $w_{i}$. Let $e_{i-1}, e_{i}$ be two consecutive Farey edges; $p$ and $p^{\prime}$ are the ends of $e_{i-1} ; p$ and $p^{\prime \prime}$ are the ends of $e_{i}$. One has $q_{i-1}^{+}=p^{\prime \prime}$ and $q_{i}^{-}=p^{\prime}$. In view of (2) and Figure 2 (top), Equation (6) translates to

$$
\begin{aligned}
-\left(\frac{\beta^{+}}{\alpha^{+}} \wedge p^{\prime \prime}\right) & <w_{i-1}<\frac{\beta^{-}}{\alpha^{-}} \wedge p^{\prime \prime} \\
-\left(\frac{\beta^{+}}{\alpha^{+}} \wedge p^{\prime}\right) & <-w_{i}<\frac{\beta^{-}}{\alpha^{-}} \wedge p^{\prime} \\
-\left(\frac{\beta^{+}}{\alpha^{+}} \wedge p\right) & <w_{i}-w_{i-1}<\frac{\beta^{-}}{\alpha^{-}} \wedge p .
\end{aligned}
$$

Using the fact that the $w_{i}$ are positive (4), this simplifies to (respectively)

$$
\begin{aligned}
w_{i-1} & <\frac{\beta^{-}}{\alpha^{-}} \wedge q_{i-1}^{+} \\
-\left(\frac{\beta^{+}}{\alpha^{+}} \wedge q_{i}^{-}\right) & <\quad-w_{i} \\
-\left(\frac{\beta^{+}}{\alpha^{+}} \wedge p\right) & <w_{i}-w_{i-1}<\frac{\beta^{-}}{\alpha^{-}} \wedge p .
\end{aligned}
$$


Finally, observe that $\frac{\beta^{+}}{\alpha^{+}} \wedge p=\left(\frac{\beta^{+}}{\alpha^{+}} \wedge q_{i-1}^{-}\right)-\left(\frac{\beta^{+}}{\alpha^{+}} \wedge q_{i}^{-}\right)$while $\frac{\beta^{-}}{\alpha^{-}} \wedge p=$ $\left(\frac{\beta^{-}}{\alpha^{-}} \wedge q_{i}^{+}\right)-\left(\frac{\beta^{-}}{\alpha^{-}} \wedge q_{i-1}^{+}\right)$by Equation (8). Therefore, if we introduce

$$
\phi_{i}^{+}:=\frac{\beta^{+}}{\alpha^{+}} \wedge q_{i}^{-} \text {and } \phi_{i}^{-}:=\frac{\beta^{-}}{\alpha^{-}} \wedge q_{i}^{+},
$$

then Equation (6) reduces to

$$
\begin{aligned}
w_{i} & <\min \left\{\phi_{i}^{+}, \phi_{i}^{-}\right\} \\
\phi_{i-1}^{-}-\phi_{i}^{-} & \left.<w_{i-1}-w_{i}<\phi_{i-1}^{+}-\phi_{i}^{+}\right\} \forall i \in \mathbb{Z} .
\end{aligned}
$$

\section{3-B. Study of $\phi^{+}$and $\phi^{-}$.}

Definition 3.1. In 1-A, we associated to each $i \in \mathbb{Z}$ a Farey edge $e_{i}$ living between two letters of $\{R, L\}$. We call $i$ a hinge index, and $\Delta_{i}$ a hinge tetrahedron, if the two letters are distinct $(R L$ or $L R)$. Non-hinges correspond to $R R$ or $L L$.

Lemma 3.2. The following properties hold concerning the two sequences $\phi^{+}, \phi^{-}: \mathbb{Z} \rightarrow \mathbb{R}_{+}^{*}$.

(i) First, $\phi^{-}$is strictly increasing and $\phi^{+}$is strictly decreasing.

(ii) For all $i$ one has $1<\phi_{i+1}^{-} / \phi_{i}^{-}<2$ and $1<\phi_{i-1}^{+} / \phi_{i}^{+}<2$.

(iii) If $i \in \mathbb{Z}$ is non-hinge, then $\phi_{i-1}^{+}+\phi_{i+1}^{+}=2 \phi_{i}^{+}$and $\phi_{i-1}^{-}+\phi_{i+1}^{-}=$ $2 \phi_{i}^{-}$.

(iv) If $i \in \mathbb{Z}$ is hinge, then $\phi_{i+1}^{-}=\phi_{i}^{-}+\phi_{i-1}^{-}$and $\phi_{i-1}^{+}=\phi_{i}^{+}+\phi_{i+1}^{+}$.

(v) The sequences $\phi^{ \pm}$are (weakly) convex, i.e., $2 \phi_{i}^{ \pm} \leq \phi_{i-1}^{ \pm}+\phi_{i+1}^{ \pm}$.

(vi) If $i<k$ are consecutive hinge indices, then $1+\frac{k-i}{2} \leq\left\{\frac{\phi_{i}^{+}}{\phi_{k}^{+}}, \frac{\phi_{k}^{-}}{\phi_{i}^{-}}\right\} \leq$ $1+k-i$.

(vii) One has $\lim _{+\infty} \phi^{+}=\lim _{-\infty} \phi^{-}=0$ and $\lim _{-\infty} \phi^{+}=\lim _{+\infty} \phi^{-}=+\infty$.

(viii) One has $\lim _{-\infty}\left(\phi_{i-1}^{+}-\phi_{i}^{+}\right)=\lim _{+\infty}\left(\phi_{i+1}^{-}-\phi_{i}^{-}\right)=+\infty$.

Remark 3.3. Identities (iii) and (iv) on $\phi^{ \pm}$are just equality cases of the inequalities (4) on $w$, which encode positivity of the angles $x_{i}, y_{i}, z_{i}$.

Proof. We will deal only with $\phi^{+}$: the arguments for $\phi^{-}$are analogous. Let $e_{i-1}=p r_{i-1}$ and $e_{i}=p r_{i}$ be consecutive Farey edges. We have $\phi_{i}^{+}=\frac{\beta^{+}}{\alpha^{+}} \wedge r_{i-1}$ while $\phi_{i-1}^{+}=\frac{\beta^{+}}{\alpha^{+}} \wedge r_{i-1}+\frac{\beta^{+}}{\alpha^{+}} \wedge p$ by Equation (8); hence (i).

For (ii), we need care only about the upper bound. Just observe that $\phi_{i-1}^{+}=\left(\frac{\beta^{+}}{\alpha^{+}} \wedge r_{i}+\frac{\beta^{+}}{\alpha^{+}} \wedge p\right)+\frac{\beta^{+}}{\alpha^{+}} \wedge p$ while $\phi_{i}^{+}=\frac{\beta^{+}}{\alpha^{+}} \wedge r_{i}+\frac{\beta^{+}}{\alpha^{+}} \wedge p$.

For (iii), assume $e_{i+1}=p r_{i+1}$ so that $\phi_{k}^{+}=\frac{\beta^{+}}{\alpha^{+}} \wedge p+\frac{\beta^{+}}{\alpha^{+}} \wedge r_{k}$ for $|i-k| \leq 1$. For $k \in\{i, i+1\}$ the right hand side is $\frac{\beta^{+}}{\alpha^{+}} \wedge r_{k-1}$, so $\left(\phi_{k-1}^{+}-\phi_{k}^{+}\right)=\frac{\beta^{+}}{\alpha^{+}} \wedge p$, which is sufficient. 
For (iv), assume $e_{i+1}=p^{\prime} r_{i}$. In the notations of Formula (9), we have $q_{i+1}^{-}=p$ and $q_{i}^{-}=r_{i-1}$, the ends of $e_{i-1}$. This together with Equation (8) yields the result. Point (v) follows from (iv) and (ii) at hinges indices, and from (iii) at other indices. For (vi), observe that $\phi_{i}^{+}=\phi_{k}^{+}+(k-i) \phi_{k+1}^{+}$by (iii)-(iv), and conclude using (ii). Point (vii) follows from (vi), and the presence of infinitely many hinge indices near either end. Point (viii) follows from (vii), (v), and (iv).

q.e.d.

3-C. Behavior of the pleatings $\lambda_{i}: \mathcal{C} \rightarrow \mathbb{R}$. For any real sequence $u \in \mathbb{R}^{\mathbb{Z}}$, define the real sequence $\nabla u$ by $(\nabla u)_{i}=u_{i-1}-u_{i}$. Let us summarize the conditions imposed on $w: \mathbb{Z} \rightarrow \mathbb{R}_{+}^{*}$ (Equations 4 and 10 above):

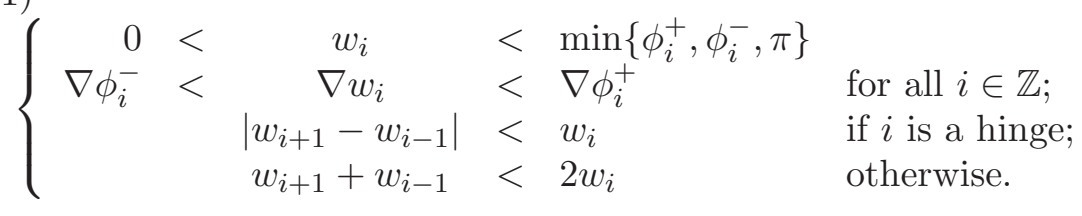

It is a simple exercise to check that $w_{i}=\tanh \phi_{i}^{+} \tanh \phi_{i}^{-}$, for instance, satisfies this system (see Chapter 3 of [G2] for detail - tanh may be replaced by any strictly concave monotonous function from $\mathbb{R}_{+}$to $[0,1)$ with the same 1-jet at the origin).

Definition 3.4. If $\left(11^{\prime}\right)$ denotes the system (11) in which all strong inequalities have been turned into weak ones, let $W \subset \mathbb{R}^{\mathbb{Z}}$ be the solution space of $\left(11^{\prime}\right)$.

Suppose $\left(w_{i}\right)_{i \in \mathbb{Z}} \in W$ and recall (5) the corresponding pleating measure $\lambda_{i}: \mathcal{C} \rightarrow \mathbb{R}$ of $S_{i}$, the pleated punctured torus between the tetrahedra $\Delta_{i}$ and $\Delta_{i-1}$.

Lemma 3.5. For any curve $\gamma \in \mathcal{C}$, the sequence $\left(\lambda_{i}(\gamma)\right)_{i \in \mathbb{Z}}$ is nondecreasing with

$$
\lambda^{-}(\gamma) \leq \lim _{i \rightarrow-\infty} \lambda_{i}(\gamma) \leq \lim _{i \rightarrow+\infty} \lambda_{i}(\gamma) \leq \lambda^{+}(\gamma)
$$

Proof. First, observe that the triples of pleating angles of $S_{i}, S_{i+1}$ are always of the form $\left(A, B,-x_{i}-y_{i}\right) ;\left(A-2 x_{i}, B-2 y_{i}, x_{i}+y_{i}\right)$ given in Figure 3, where $x_{i}$ (resp. $y_{i}$ ) is positive, equal to the interior dihedral angle of the tetrahedron $\Delta_{i}$ at the horizontal (resp. vertical) edges of the (square) fundamental domain of the surface $S$. The closed curve $\gamma$ in $S$ traverses that fundamental domain a number of times, either vertically, or horizontally, or diagonally (cutting off one of the four corners). The pleating along $\gamma$ increases by $2 y_{i}$ per vertical passage, $2 x_{i}$ per horizontal passage, and 0 per diagonal passage; hence the monotonicity statement. (This argument, or a variant of it, is also valid for higher-genus surfaces 

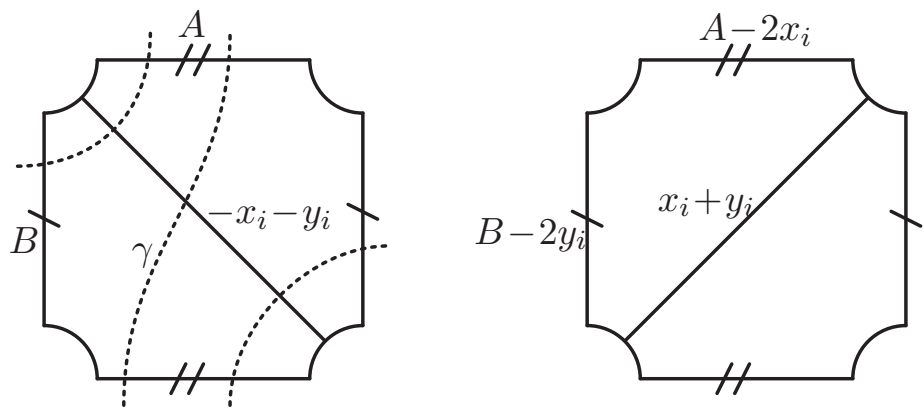

Figure 3. The pleatings of the surfaces $S_{i}$ and $S_{i+1}$ bounding $\Delta_{i}$.

and non-simple closed curves, as long as the tetrahedron has positive angles. More detail is given in [G2].)

For the bounding, we will focus only on the $\lambda^{+}$-side. Consider the slope $\sigma=\frac{\eta}{\xi} \in \mathbb{P}^{1} \mathbb{Q}$ of $\gamma$ (a reduced fraction); recall the definition $\lambda^{+}(\sigma)=\left\|_{\alpha^{+} \xi}^{\beta^{+}}\right\|$from (1). Consider a large enough index $i$, such that the Farey edge $e_{i-1}$ separates $\sigma$ from $\beta^{+} / \alpha^{+}$. Consider the points $p, p^{\prime}, p^{\prime \prime} \in$ $\mathbb{P}^{1} \mathbb{Q}$ such that $\left(e_{i-1}, e_{i}\right)=\left(p p^{\prime}, p p^{\prime \prime}\right)$ : the points $\left(\sigma, p, \frac{\beta^{+}}{\alpha^{+}}, p^{\prime \prime}, p^{\prime}, \sigma\right)$ are cyclically arranged in $\mathbb{P}^{1} \mathbb{R}$. Observe that the $\wedge$-notation (7) applied at rationals is just the (geometric) intersection number of the corresponding curves. Therefore, using the angle information given in Figure 2 (top), one has

$$
\begin{aligned}
\lambda_{i}(\gamma) & =(p \wedge \sigma)\left(w_{i}-w_{i-1}\right)-\left(p^{\prime} \wedge \sigma\right) w_{i}+\left(p^{\prime \prime} \wedge \sigma\right) w_{i-1} \\
& =w_{i}(p \wedge \sigma)+\nabla w_{i}\left(p^{\prime} \wedge \sigma\right) \quad \text { since } p^{\prime \prime} \wedge \sigma=p \wedge \sigma+p^{\prime} \wedge \sigma \\
& \leq \phi_{i}^{+}(p \wedge \sigma)+\nabla \phi_{i}^{+}\left(p^{\prime} \wedge \sigma\right) \\
& =\left(\frac{\beta^{+}}{\alpha^{+}} \wedge p^{\prime}\right)(p \wedge \sigma)+\left(\frac{\beta^{+}}{\alpha^{+}} \wedge p\right)\left(p^{\prime} \wedge \sigma\right) \quad(*)
\end{aligned}
$$

by definition (9) of $\phi^{+}$. The last quantity is $\frac{\beta^{+}}{\alpha^{+}} \wedge \sigma$ (hence the upper bound): indeed, by $S L_{2}$-invariance of the $\wedge$-notation, it is enough to check it when $p=\infty$ and $p^{\prime}=0$ - in that case, $\frac{\beta^{+}}{\alpha^{+}}$and $\sigma=\frac{\eta}{\xi}$ have opposite signs, and $(*)$ does, indeed, become $\left|\beta^{+} \xi\right|+\left|\alpha^{+} \eta\right|=\| \begin{array}{cc}\beta^{+} & \eta \\ \alpha^{+} & \xi\end{array} \mid$.

\section{Hyperbolic volume}

The product topology on $\mathbb{R}^{\mathbb{Z}}$ induces a natural topology on the space $W$ of Definition 3.4: clearly, $W$ is nonempty, convex, and compact.

If $(x, y, z)$ is a nonnegative triple such that $x+y+z=\pi$, let $\mathcal{V}(x, y, z)$ be the hyperbolic volume of an ideal tetrahedron whose interior dihedral 
angles are $x, y, z$. We wish to compute the total hyperbolic volume of all tetrahedra when $w \in W$, i.e.,

$$
\mathcal{V}(w):=\sum_{i \in \mathbb{Z}} \mathcal{V}\left(x_{i}, y_{i}, z_{i}\right)
$$

where $x_{i}, y_{i}, z_{i}$ are defined from the $w_{i}$ via Table (3). This poses the problem of well-definedness - the sum of the volumes might diverge. Let us estimate $\mathcal{V}$ : a well-known explicit formula [Mil] gives

$$
\begin{aligned}
\mathcal{V}(x, y, z) & =-\int_{0}^{x} \log 2 \sin -\int_{0}^{y} \log 2 \sin -\int_{0}^{z} \log 2 \sin \\
& =\int_{0}^{x} \log \frac{\sin (\tau+y)}{\sin \tau} d \tau \quad\left(\text { as } \int_{0}^{\pi} \log 2 \sin =0\right) \\
& \left.\leq \int_{0}^{x} \log \frac{\tau+y}{\tau} d \tau \quad \text { (by concavity of } \sin \right) \\
& =x \log \frac{x+y}{x}+y \log \frac{x+y}{y} \\
& \leq(x+y) \log 2 \quad \text { (concavity of } \log ) .
\end{aligned}
$$

Lemma 4.1. There exists a universal constant $K>0$ such that the sum of the volumes of the tetrahedra $\Delta_{j}$ for $j \geq i$ (resp. $j \leq i$ ) is at most $K \phi_{i}^{+}\left(\right.$resp. $\left.K \phi_{i}^{-}\right)$.

Proof. We will focus only on the $\phi^{+}$-statement. First, by the computation above, the volume of the tetrahedron $\Delta_{i}$ is at most $w_{i} \log 2 \leq$ $\phi_{i}^{+} \log 2$ (see Table 3). In view of Lemma 3.2-(vi), this implies that the total volume of all hinge tetrahedra beyond the index $i$ is at most $3 \phi_{i}^{+} \log 2$. For the same reason, it is sufficient to prove

Sublemma 4.2. There exists a universal constant $L>0$ such that if 0 and $N \in \mathbb{N}$ are two consecutive hinge indices, then the sum of the volumes of the tetrahedra $\Delta_{1}, \Delta_{2}, \ldots, \Delta_{N-1}$ is at most $L \phi_{0}^{+}$.

Proof. In view of homogeneity in the estimation (13), it is sufficient to assume $\phi_{0}^{+}=1$ and replace the volume with its estimate. Therefore, let $\left(w_{i}\right)_{0 \leq i \leq N}$ be a concave sequence in $[0,1]$ : following Table $(3)$, we want to find a universal upper bound $L$ (not depending on $N$ ) for

$$
\begin{aligned}
\sum_{i=1}^{N-1} \frac{w_{i+1}+w_{i-1}}{2} \log & \frac{2 w_{i}}{w_{i+1}+w_{i-1}} \\
& +\frac{2 w_{i}-w_{i+1}-w_{i-1}}{2} \log \frac{2 w_{i}}{2 w_{i}-w_{i+1}-w_{i-1}} .
\end{aligned}
$$

If $A<B$ are positive integers, denote by $\Sigma_{A}^{B}$ the restriction of the above sum to indices $A \leq i<B$. Observe that the general term of $\Sigma_{A}^{B}$ is bounded by $2 e^{-1}$ because $w_{i} \leq 1$ and $\tau \log \frac{1}{\tau} \leq e^{-1}$ for all positive $\tau$. 
For yet better control, we bound the first half of the general term of $\Sigma_{A}^{B}$ by $\left(\frac{w_{i+1}+w_{i-1}}{2}\right)\left(\frac{2 w_{i}}{w_{i+1}+w_{i-1}}-1\right.$ ), and the second half by concavity of $\log$. This produces

$$
\begin{aligned}
\Sigma_{A}^{B} \leq & \sum_{i=A}^{B-1}\left(\frac{w_{i+1}+w_{i-1}}{w_{i+1}+w_{i-1}} \times \frac{2 w_{i}-w_{i+1}-w_{i-1}}{2}\right) \\
& +\left(\sum_{i=A}^{B-1} \frac{2 w_{i}-w_{i+1}-w_{i-1}}{2}\right) \log \frac{\sum_{i=A}^{B-1} w_{i}}{\sum_{i=A}^{B-1} \frac{2 w_{i}-w_{i+1}-w_{i-1}}{2}} \\
= & \sigma \log \frac{e \sum_{i=A}^{B-1} w_{i}}{\sigma}, \quad \text { where } \sigma=\frac{w_{A}-w_{A-1}+w_{B-1}-w_{B}}{2} .
\end{aligned}
$$

Denote by $M \in \llbracket 0, N \rrbracket$ a value of the index $i$ for which $w_{i}$ is maximal. If $A<B \leq M$, we have

$$
0 \leq \sigma \leq \frac{w_{A}-w_{A-1}}{2} \leq \frac{1}{2 A} \leq 1
$$

(the third inequality follows from concavity of $w$ between 0 and $A$ ). Also notice that $f: \tau \mapsto \tau \log \frac{e}{\tau}$ is nondecreasing on $[0,1]$. We shall apply these facts for $A=2^{k-1}$ and $B=\min \left\{2^{k}, M\right\}$ : the previous bound on $\Sigma_{A}^{B}$ can be rewritten

$$
\begin{aligned}
\Sigma_{A}^{B} & \leq f(\sigma)+\sigma \log \left(\sum_{i=A}^{B-1} w_{i}\right) \\
& \leq f\left(2^{-k}\right)+2^{-k} \log 2^{k-1}=2^{-k}[1+(2 k-1) \log 2] .
\end{aligned}
$$

The latter numbers (for $k$ ranging over $\mathbb{N}^{*}$ ) add up to some universal $L^{\prime}<+\infty$. After a similar argument for the indices $M<i<N$, we can take $L=2 e^{-1}+2 L^{\prime}$.

q.e.d.

Finally, we can take $K=3 L+3 \log 2$. Lemma 4.1 is proved. q.e.d.

Corollary 4.3. The volume functional $\mathcal{V}: W \rightarrow \mathbb{R}^{+}$is well-defined, continuous, and concave.

Proof. Well-definedness is the point of Lemma 4.1. Given $\varepsilon>0$, only finitely many indices $i$ satisfy $\min \left\{\phi_{i}^{+}, \phi_{i}^{-}\right\}>\varepsilon / K$, and the others contribute at most $2 \varepsilon$ to the volume: hence continuity in the product topology. Concavity follows from the concavity of the volume of one tetrahedron (parametrized by its angles): see e.g. Proposition 8 of [GF].

q.e.d.

Therefore, by compactness, there exists a sequence $\left(w_{i}\right)_{i \in \mathbb{Z}} \in W$, which maximizes the hyperbolic volume $\mathcal{V}$. From this point on, $w$ will denote that maximizer.

Proposition 4.4. For each $j \in \mathbb{Z}$, if $x_{j} y_{j} z_{j}=0$, then $\max \left\{x_{j}, y_{j}, z_{j}\right\}$ $=\pi$. 
Proof. Assume the tetrahedron $\Delta_{j}$ has exactly one vanishing angle, and aim for a contradiction. If $\mathcal{V}\left(\Delta^{t}\right)$ is the volume of a tetrahedron $\Delta^{t}$ having angles $x^{t}, y^{t},\left(\pi-x^{t}-y^{t}\right)$ with $\left(x^{t}, y^{t}\right)_{t \geq 0}$ smooth, $x^{0}=0<$ $y^{0}<\pi$ and $d x^{t} / d t_{\mid t=0}>0$, then $d \mathcal{V}\left(\Delta^{t}\right) / d t_{\mid t=0}=+\infty$ (by Formula (13) above).

Let $\left(w_{i}^{\prime}\right)_{i \in \mathbb{Z}}$ be a sequence satisfying all (strict) inequalities of (11) and define $w^{t}:=w+t\left(w^{\prime}-w\right)$ for $0 \leq t \leq 1$. Denote by $\Delta_{i}^{t}$ the $i$-th tetrahedron determined via $(3)$ by $\left(w_{i}^{t}\right)_{i \in \mathbb{Z}}$ : then the angles of $\Delta_{j}^{t}$ satisfy the hypotheses of the previous paragraph, so

$$
\mathcal{V}\left(w^{t}\right)=\mathcal{V}\left(\Delta_{j}^{t}\right)+\left(\sum_{i \neq j} \mathcal{V}\left(\Delta_{i}^{t}\right)\right)
$$

has right derivative $+\infty$ at $t=0$ (the second summand is concave and continuous, so it has a well-defined right derivative in $\mathbb{R} \cup\{+\infty\}$ at 0 ). Therefore, $\mathcal{V}$ was not maximal at $w$.

q.e.d.

In Section 10, we will need the following consequence of Proposition 4.4:

Proposition 4.5. For any integer $j \in \mathbb{Z}$, if $x_{j} y_{j} z_{j}=0$, then $j$ is a hinge index and $w_{j}=0$.

This is Proposition 13 in $[\mathbf{G F}]$. But, in fact, much more is true:

Proposition 4.6. All the (strict) inequalities of (11) are true at $w$.

Proof. If some inequality of (11) involving $\phi^{+}$fails to be strict, it is easy to see by induction from (11) that $w=\phi^{+}$near $+\infty$, so all tetrahedra $\Delta_{i}$ (for $i$ large enough) have exactly one vanishing angle (see Remark 3.3): $w$ was not maximal by Proposition 4.4. Therefore, all inequalities of (11) involving $\phi^{ \pm}$are strict. The arguments in [GF] (especially Lemma 16 and the argument of Section 9 there) can then be copied verbatim to show that all inequalities (11) are strict at $w$, so $w$ is a critical point of the volume functional $\mathcal{V}$.

q.e.d.

Proposition 4.6 has two important consequences: first, the volume maximizer $\left(w_{i}\right)_{i \in \mathbb{Z}}$ is unique because the volume of an ideal tetrahedron with strictly positive angles is a strictly concave function of these angles. Second, the holonomy representation is trivial, i.e., the gluing of any finite number of consecutive tetrahedra $\Delta_{i}$ defines a complete hyperbolic metric with (polyhedral) boundary (the shapes of the $\Delta_{i}$ "fit together correctly" around the edges): see $[\mathbf{R i}],[\mathbf{C H}]$ or $[\mathbf{G F}]$. Therefore, the links of the vertices of the ideal tetrahedra (Euclidean triangles) form a triangulation of the link of the puncture: the latter is naturally endowed with a Euclidean structure and its universal cover can be drawn in the plane (Figure 4 - more on the combinatorics of this triangulation 
in Section 6; see also $[\mathbf{G F}])$. Denote by $\Gamma$ the image of the induced holonomy representation $\pi_{1}(S) \rightarrow \operatorname{Isom}^{+}\left(\mathbb{H}^{3}\right)$.

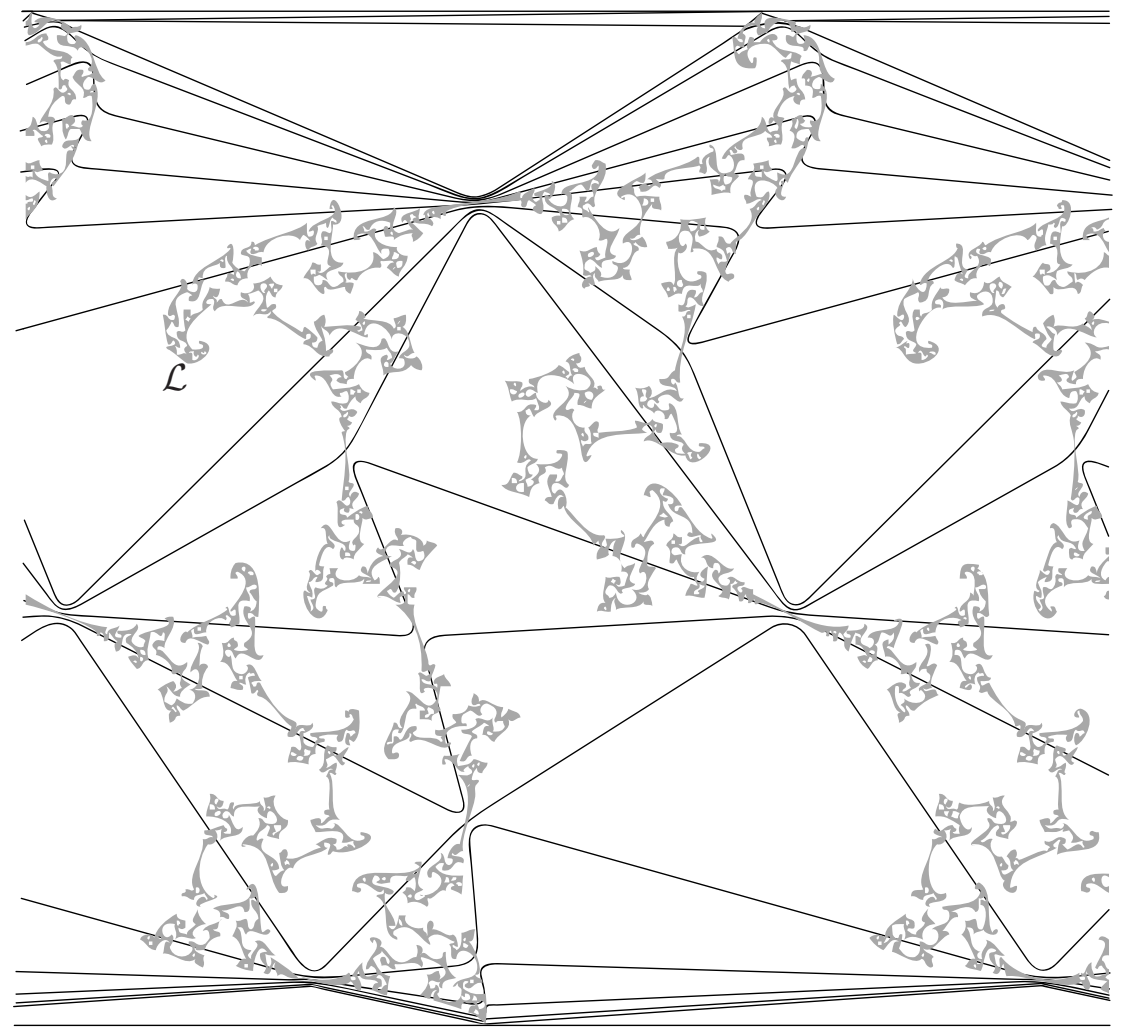

Figure 4. The cusp triangulation is shown, in anticipation, against the limit set $\mathcal{L} \subset \mathbb{P}^{1} \mathbb{C}$ of the quasifuchsian group (a complicated Jordan curve). The picture extends periodically to the right and left. Each broken line is the puncture link of a pleated surface $S_{i}$; the vertices (whose design artificially sets the $S_{i}$ apart from each other) are all parabolic fixed points at which the limit set $\mathcal{L}$ becomes pinched. Infinitely many very flat triangles accumulate along the top and bottom horizontal lines. This picture was made using Masaaki Wada's software Opti [Wa].

\section{Behavior of $\left(w_{i}\right)$}

We want to prove that the pleatings $\lambda_{i}$ (see Section 3 ) of the pleated punctured tori $S_{i}$ converge (in the weak-* topology, i.e., on any test curve $\gamma \in \mathcal{C}$ ) to the pleatings $\lambda^{ \pm}$near infinity. For this we must study $\left(w_{i}\right)$, and especially show that the bounds specified by $\phi^{ \pm}$in Equations 
(11) are almost (but not quite) achieved. We use the $\nabla$-notation as in Section 3-C.

Lemma 5.1. Recall that $\nabla \phi^{-}<0<\nabla \phi^{+}$(Lemma 3.2). One has

$$
\max \left\{\varlimsup_{\mathbb{Z}} \frac{w}{\phi^{+}}, \varlimsup_{\mathbb{Z}} \frac{\nabla w}{\nabla \phi^{+}}\right\}=\max \left\{\varlimsup_{\mathbb{Z}} \frac{w}{\phi^{-}}, \varlimsup_{\mathbb{Z}} \frac{-\nabla w}{-\nabla \phi^{-}}\right\}=1 .
$$

Proof. We focus on the $\phi^{+}$-statement; the $\phi^{-}$-part is analogous. Since $\frac{w}{\phi^{+}}<1$, assuming $\varlimsup \frac{w}{\phi^{+}}<1$ implies sup $\frac{w}{\phi^{+}}<1$, and the same holds true for $\frac{\nabla w}{\nabla \phi^{+}}$(see Equation 11). Therefore, suppose sup $\frac{w}{\phi^{+}} \leq 1-\varepsilon$ and $\sup \frac{\nabla w}{\nabla \phi^{+}} \leq 1-\varepsilon$ for some $\varepsilon>0$, and aim at a contradiction.

Recall the ordered pair $\left(\alpha^{+}, \beta^{+}\right)$that helped define $\phi^{+}$. For each $\mu>0$, define $\left(\alpha^{\mu}, \beta^{\mu}\right):=\left(\mu \alpha^{+}, \mu \beta^{+}\right)$. This defines a new pleating function $\lambda^{\mu}=\mu \lambda^{+}: \mathcal{C} \rightarrow \mathbb{R}^{+}$, a new $\phi^{\mu}=\mu \phi^{+}$, and a new domain $W^{\mu}$ by (11) (the numbers $\alpha^{-}$and $\beta^{-}$are left unchanged). By definition, $W=W^{1}$ and $W^{\mu} \subset W^{\mu^{\prime}}$ if and only if $\mu \leq \mu^{\prime}$. Let $\left(w_{i}^{\mu}\right)_{i \in \mathbb{Z}}$ be the maximizer of the volume functional $\mathcal{V}$ on $W^{\mu}$. By assumption, we have $w^{1} \in W^{1-\varepsilon} \subset W^{1}$, so $w^{1}=w^{1-\varepsilon}$.

Write $V(\mu)=\mathcal{V}\left(w^{\mu}\right)$, so that $V(1)=V(1-\varepsilon)$. It is straightforward to check that for any $t \in[0,1]$, one has $\left(t \cdot w^{\mu}+(1-t) \cdot w^{\mu^{\prime}}\right) \in W^{t \mu+(1-t) \mu^{\prime}}$. Since the volume of any tetrahedron is a concave function of its angles, this is enough to imply that $V: \mathbb{R}^{+} \rightarrow \mathbb{R}^{+}$is (weakly) concave. By inclusion, $V$ is also nondecreasing. In fact, $V$ is strictly increasing (which will finish the proof by contradiction): to prove this, since $V$ is concave, we just need to produce arbitrarily large values of the volume $\mathcal{V}$ at points $v^{\mu} \in W^{\mu}$, for large enough $\mu$. We may assume (up to a translation of indices, see Lemma 3.2, (vii)-(viii)) that $\phi_{0}^{-}>\pi$ and $-\nabla \phi_{0}^{-}>\pi$. Then, start by defining $v_{i}^{\mu}=\min \left\{\phi_{i}^{\mu}, \phi_{i}^{-}, \pi\right\}$, so that $v^{\mu}=\pi$ on $\llbracket 0, N+$ 1』 for arbitrarily large $N$. By just taking $\mu$ large enough, we may further assume $\nabla \phi^{\mu}>\pi$ on $\llbracket 0, N+1 \rrbracket$. Then, each time $i \in \llbracket 1, N \rrbracket$ is a hinge index, replace $v_{i}^{\mu}$ by $2 \pi / 3$ : this is allowed in $W^{\mu}$ by our assumptions on $\nabla \phi^{-}, \nabla \phi^{\mu}$. The angles of the tetrahedron $\Delta_{i}$ are then all in $\{\pi / 2, \pi / 3, \pi / 6\}$ (see Table 3 ). Since there are infinitely many hinge indices near $+\infty$, the volume can become arbitrarily large: Lemma 5.1 is proved.

q.e.d.

Corollary 5.2. In fact,

$$
\varlimsup_{i \rightarrow+\infty} \min \left\{\frac{w_{i}}{\phi_{i}^{+}}, \frac{\nabla w_{i}}{\nabla \phi_{i}^{+}}\right\}=\varlimsup_{i \rightarrow-\infty} \min \left\{\frac{w_{i-1}}{\phi_{i-1}^{-}}, \frac{-\nabla w_{i}}{-\nabla \phi_{i}^{-}}\right\}=1 .
$$

Proof. Again, we focus only on $\phi^{+}$. By Lemma 5.1, there exists a subsequence $\left(w_{\nu(i)}\right)_{i \in \mathbb{N}}$ such that $w_{\nu(i)} \sim \phi_{\nu(i)}^{+}$or $\nabla w_{\nu(i)} \sim \nabla \phi_{\nu(i)}^{+}$. Suppose the latter is the case. For an arbitrary integer $i$, let $n$ be the smallest hinge index larger than or equal to $\nu(i)$ : observe that $\phi_{n+1}^{+}=$ 
$\nabla \phi_{n}^{+}=\nabla \phi_{\nu(i)}^{+}$by Lemma 3.2 (iii)-(iv), while $w_{n+1} \geq \nabla w_{n} \geq \nabla w_{\nu(i)}$ by the positivity conditions (11). Therefore, $\frac{w_{n+1}}{\phi_{n+1}^{+}} \geq \frac{\nabla w_{\nu(i)}}{\nabla \phi_{\nu(i)}^{+}}$, so up to redefining $\nu$, we may assume simply $w_{\nu(i)} \sim \phi_{\nu(i)}^{+}$.

Pick $\varepsilon>0$. Take $i$ such that

$$
w_{\nu(i)} \geq(1-\varepsilon) \phi_{\nu(i)}^{+} .
$$

Let $n$ be the smallest hinge index strictly larger than $\nu(i)$. If $n=\nu(i)+1$, then

$$
w_{n} \geq w_{n-1}-\nabla \phi_{n}^{+} \geq(1-\varepsilon) \phi_{n-1}^{+}-\phi_{n+1}^{+}=\phi_{n}^{+}-\varepsilon \phi_{n-1}^{+} \geq(1-2 \varepsilon) \phi_{n}^{+}
$$

and

$$
\begin{aligned}
\nabla w_{n} & =w_{n-1}-w_{n} \geq(1-\varepsilon) \phi_{n-1}^{+}-\phi_{n}^{+}=\phi_{n+1}^{+}-\varepsilon \phi_{n-1}^{+} \\
& \geq(1-4 \varepsilon) \phi_{n+1}^{+}=(1-4 \varepsilon) \nabla \phi_{n}^{+}
\end{aligned}
$$

where Lemma 3.2 has been used several times. Therefore, $\min \left\{\frac{w_{n}}{\phi_{n}^{+}}, \frac{\nabla w_{n}}{\nabla \phi_{n}^{+}}\right\}$ $\geq 1-4 \varepsilon$.

If $n \geq \nu(i)+2$, we can find an index $k \in(\nu(i), n)$ such that $\frac{k-\nu(i)}{n-\nu(i)} \in$ $\left[\frac{1}{2}, \frac{2}{3}\right]$. We will show that $\min \left\{\frac{w_{k}}{\phi_{k}^{+}}, \frac{\nabla w_{k}}{\nabla \phi_{k}^{+}}\right\} \geq 1-8 \varepsilon$, which will finish the proof.

- By positivity of $w$ and concavity of $w$ between the points $(\nu(i), k, n)$, one has $w_{k} \geq \frac{1}{3} w_{\nu(i)}$. Therefore,

$$
\phi_{k}^{+}-w_{k} \leq \phi_{\nu(i)}^{+}-w_{\nu(i)} \leq\left(\frac{1}{1-\varepsilon}-1\right) w_{\nu(i)} \leq \frac{3 \varepsilon}{1-\varepsilon} w_{k}
$$

(here the first inequality holds because $\left(\phi^{+}-w\right)$ is decreasing by Condition (11), and the second follows from the assumption (14) above). Hence,

$$
\frac{w_{k}}{\phi_{k}^{+}} \geq \frac{1-\varepsilon}{1+2 \varepsilon} \geq 1-3 \varepsilon
$$

- Observe that

$$
(1-\varepsilon) \phi_{\nu(i)}^{+} \leq w_{\nu(i)} \leq w_{k}+(k-\nu(i)) \nabla w_{k} \leq \phi_{k}^{+}+(k-\nu(i)) \nabla w_{k}
$$

where the second inequality follows from concavity of $w$ between the points $(\nu(i), k-1, k)$. It follows that

$$
(k-\nu(i)) \nabla w_{k} \geq \phi_{\nu(i)}^{+}-\phi_{k}^{+}-\varepsilon \phi_{\nu(i)}^{+}=(k-\nu(i)) \nabla \phi_{k}^{+}-\varepsilon \phi_{\nu(i)}^{+} ;
$$

hence

$$
\nabla w_{k} \geq \nabla \phi_{k}^{+}-\frac{\varepsilon}{k-\nu(i)} \phi_{\nu(i)}^{+} \geq \nabla \phi_{k}^{+}-\frac{2 \varepsilon}{n-\nu(i)} \phi_{\nu(i)}^{+} .
$$

Since Lemma 3.2-(vi) gives us $\phi_{\nu(i)}^{+} \leq 2(n-\nu(i)) \phi_{n}^{+}$, this yields

$$
\nabla w_{k} \geq \nabla \phi_{k}^{+}-4 \varepsilon \phi_{n}^{+} \geq \nabla \phi_{k}^{+}-8 \varepsilon \phi_{n+1}^{+}=(1-8 \varepsilon) \nabla \phi_{k}^{+} .
$$


q.e.d.

Corollary 5.3. Recall the pleating $\lambda_{i}$ of the pleated surface $S_{i}$. For any simple closed curve $\gamma \in \mathcal{C}$, we have

$$
\lim _{i \rightarrow+\infty} \lambda_{i}(\gamma)=\lambda^{+}(\gamma) \text { and } \lim _{i \rightarrow-\infty} \lambda_{i}(\gamma)=\lambda^{-}(\gamma)
$$

Proof. By Corollary 5.2, the member ratio in the inequality (12) can be made arbitrarily close to 1 .

q.e.d.

\section{The cusp link}

We now aim to investigate the behavior of the pleated surfaces $S_{i}$ as $i$ goes to $\pm \infty$ - or, more precisely, to find two limiting pleated surfaces $S_{ \pm \infty}$ with pleatings $\lambda^{ \pm}$such that $\bar{V}=\bigcup_{i \in \mathbb{Z}} \Delta_{i} \sqcup S_{+\infty} \sqcup S_{-\infty}$ is metrically complete with locally convex boundary. The difficult part is to prove that the intrinsic moduli of the $S_{i}$ converge in Teichmüller space. This question will be addressed in the next section. In this section, we just describe the cusp link, introduce notation, and prove a few inequalities.

As always, we will mainly work near $+\infty$. Define $V=\bigcup_{i \in \mathbb{Z}} \Delta_{i}$. We begin by orienting all the edges of $V$ in a way that will be consistent with the pleating data $\left(\alpha^{ \pm}, \beta^{ \pm}\right)$from $(1)$. Namely, denote by $\mathcal{E} \subset \mathbb{P}^{1} \mathbb{Q}$ the collection of all the endpoints of all the Farey edges $\left(e_{i}\right)_{i \in \mathbb{Z}}$. For $\sigma \in \mathcal{E}$, let $Q_{\sigma}$ be the edge of $V$ of slope $\sigma$. Recall that the punctured torus is defined as $\left(\mathbb{R}^{2} \backslash \mathbb{Z}^{2}\right) / \mathbb{Z}^{2}$. Orienting $Q_{\sigma}$ is therefore equivalent to orienting the line $F_{\sigma}$ of slope $\sigma$ in $\mathbb{R}^{2}$. We decide that the positive half of $F_{\sigma}$ should be on the same side of the line $\mathbb{R}\left(\alpha^{-}, \beta^{-}\right)$as $\left(\alpha^{+}, \beta^{+}\right)$, and orient $Q_{\sigma}$ accordingly.

Let us now describe the link of the puncture, or cusp triangulation (whose underlying space is a Euclidean annulus $\mathbb{S}^{1} \times \mathbb{R}$ : we refer to $\mathbb{R}$ as the "vertical" direction). Each tetrahedron $\Delta_{i}$ contributes four similar Euclidean triangles at infinity to the link of the puncture corresponding to the four ideal vertices of $\Delta_{i}$. The bases of these four triangles form a closed $\mathbb{S}^{1}$-homotopic curve which is a broken line of four segments, and the triangles point alternatively up and down from this broken line (see Figure 5). The two upward (resp. downward) pointing triangles have the same Euclidean size, an effect of the hyperelliptic involution (rotation of $180^{\circ}$ around the puncture), which acts isometrically on $V$ (reversing all edge orientations) and acts as a horizontal translation on the cusp link.

Definition 6.1. Henceforth, we normalize the Euclidean scale of the cusp link so that the loop around the cusp has length 4 . We let $b_{i}$ (resp. $b_{i}^{\prime}$ ) be the length of the base of a downward-pointing (resp. upwardpointing) triangle contributed by the tetrahedron $\Delta_{i}$, and define $\chi_{i}=$ $b_{i}^{\prime} / b_{i}$ (see e.g. Figure 7 ). 


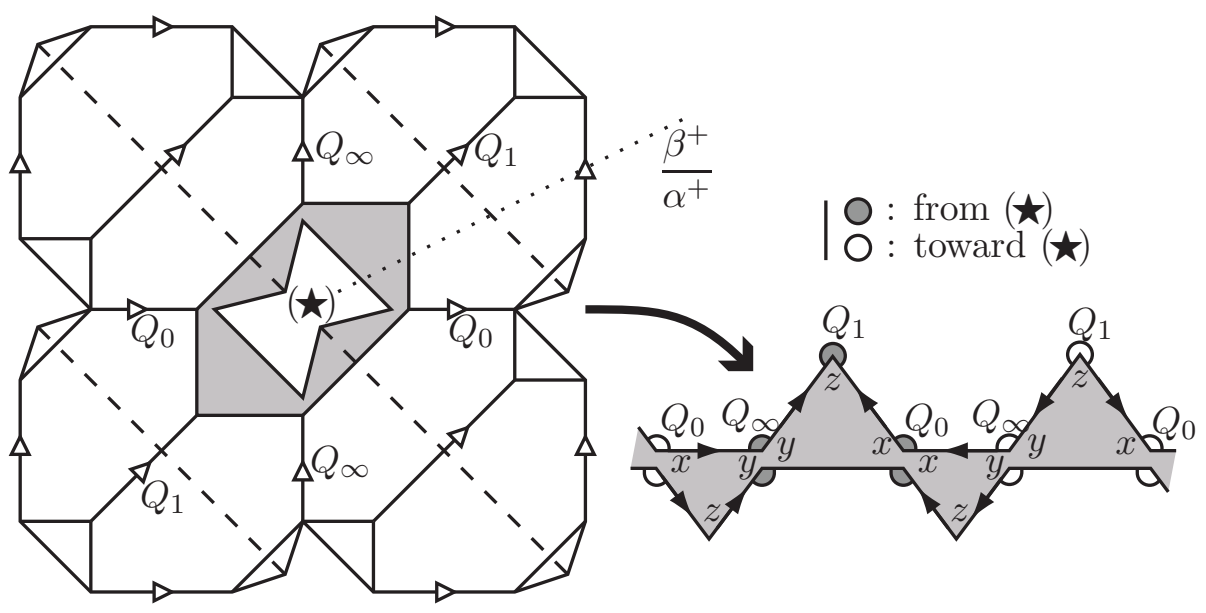

Figure 5. In the left panel, $(\star)$ marks the cusp. In the right panel, the cusp is at infinity.

Figure 5 also shows some additional information, assuming that $e_{i}=$ $0 \infty$ and $\alpha^{+}, \beta^{+}>0$ (hence $\beta^{-} / \alpha^{-}<0$ ). Namely, the pleated surfaces $S_{i+1}$ and $S_{i}$ (above and below $\Delta_{i}$ ) are pleated along $Q_{0}, Q_{\infty}, Q_{1}$ and $Q_{0}, Q_{\infty}, Q_{-1}$ respectively, and the orientations of the $Q_{\sigma}$ are as shown in the left panel of Figure 5 (the orientation of the edge $Q_{-1}$ is not determined, as it depends on the position of $\beta^{-} / \alpha^{-}$relative to -1 ). The orientations of the lines to/from the puncture are also shown in the right panel, at the vertices, with the help of a color code. Moreover, each segment $\epsilon$ of the upper broken line in the right panel corresponds to an arc about a vertex $v$ of a face $f$ of $S_{i+1}$ in the left panel, so $\epsilon$ receives the orientation of the edge of $f$ opposite $v$. If $\tau$ is one of the upward-pointing triangles drawn in the plane $\mathbb{C}$ (right panel), consider the tetrahedron $\Delta$ whose vertices are $\infty$ and those of $\tau$ : all edges of $\Delta$, except one, receive orientations from the construction above, and $\Delta$ is isometric (respecting these orientations) to $\Delta_{i}$. Finally, notice the labels in the 3 corners of each triangle in the right panel: the corner of the free vertex is labeled $z$ (the angle there being $z_{i}$ ); the other two corners are labeled $x$ and $y$ accordingly. The labels $x-y-z$ appear clockwise in each triangle.

The contribution of the tetrahedron $\Delta_{i-1}$ to the cusp triangulation is also a union of four triangles bounded by two broken lines. Moreover, the upper broken line from $\Delta_{i-1}$ is the lower broken line from $\Delta_{i}$, and the orientations of the lines to/from infinity must agree. Inspection shows that there are only two possibilities, corresponding to whether the letter living on the surface $S_{i}$ is $R$ or $L$ : for $R$, the $x$-corners of the two 4-triangle families live near a pair of common vertices $C, C^{\prime}$; whereas for $L$, the same is true of the $y$-corners (Figure 6). 


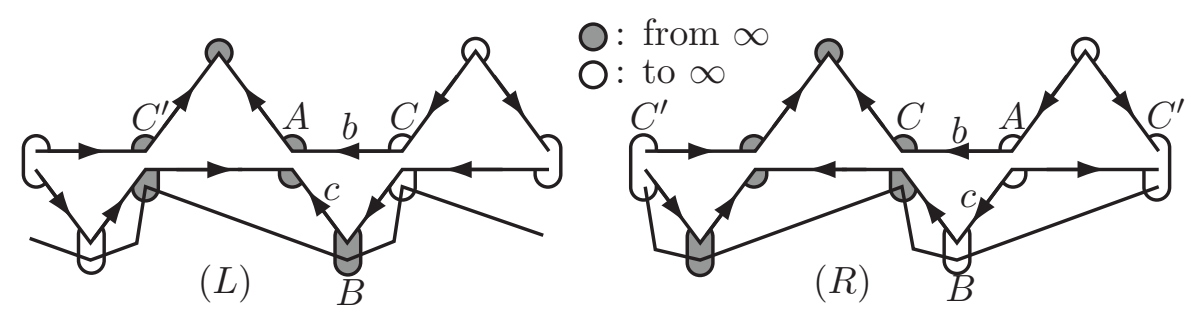

Figure 6. Left and Right transitions. Compare with the right panel of Figure 5.

Definition 6.2. In a downward-pointing triangle defined by $\Delta_{i}$, the edge lengths are $b_{i}$ (the basis from Def. 6.1), $b_{i-1}$, and a third number which we call $c_{i}$. In either panel of Figure 6 , we thus have $b=b_{i} ; c=c_{i}$ and $B C=b_{i-1}$, where the tetrahedron $\Delta_{i}$ defines the top four triangles.

Property 6.3. For $i$ large enough, $\left(b_{i}\right)$ is increasing and $\left(b_{i}^{\prime}\right)$ is decreasing.

Proof. Let $\tau_{i}$ (resp. $\tau_{i}^{\prime}$ ) be a downward-pointing (resp. upwardpointing) triangle defined by the tetrahedron $\Delta_{i}$. For large enough $i$ we have $z_{i}=\pi-w_{i}>\pi-\phi_{i}^{+}>\pi / 2$, so $b_{i}$ is the longest edge of $\tau_{i}$, and $b_{i}^{\prime}$ the longest edge of $\tau_{i}^{\prime}$. Since $b_{i-1}$ is an edge of $\tau_{i}$ and $b_{i+1}^{\prime}$ is an edge of $\tau_{i}^{\prime}$ (Figure 6 or 7 ), the conclusion follows.

q.e.d.

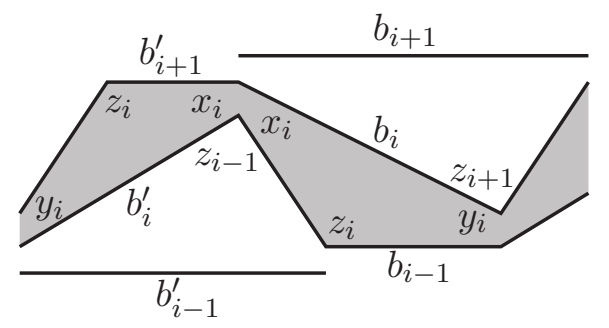

Figure 7. Here the index $i$ is a hinge; the nature of $i \pm 1$ is undetermined.

Property 6.4. We have $\lim _{i \rightarrow+\infty} \chi_{i}=0$ (see Definition 6.1).

Proof. We already know that $\left(\chi_{i}\right)=\left(b_{i}^{\prime} / b_{i}\right)$ is ultimately decreasing. It is therefore enough to show that $\chi_{i+1} / \chi_{i-1} \leq 1 / 2$ for large enough hinge indices $i$. Consider Figure 7, where angles labeled $z$ are obtuse (as a rule, we shade a cusp triangle contributed by $\Delta_{i}$ whenever $i$ is a hinge index). Check that

$$
\frac{\chi_{i+1}}{\chi_{i-1}}=\frac{b_{i+1}^{\prime}}{b_{i-1}^{\prime}} \frac{b_{i-1}}{b_{i+1}}<\frac{b_{i+1}^{\prime}}{b_{i}^{\prime}} \frac{b_{i-1}}{b_{i}}=\frac{\sin x_{i} \sin y_{i}}{\sin ^{2} z_{i}} \leq \frac{\sin ^{2}\left(w_{i} / 2\right)}{\sin ^{2} w_{i}}<\frac{1}{2}
$$


(the last two inequalities follow from an easy study of sin, using $x_{i}+y_{i}=$ $\left.\pi-z_{i}=w_{i}<\pi / 2\right)$.

q.e.d.

As an immediate consequence, we find $\lim _{+\infty} b_{i}^{\prime}=0$ and $\lim _{+\infty} b_{i}=$ 2. Similarly, $\lim _{-\infty} b_{i}^{\prime}=2$ and $\lim _{-\infty} b_{i}=0$. Compare this with the metrically accurate Figure 4 on p. 106.

Definition 6.5. Let $J \subset \mathbb{Z}$ be the set of all integers $j$ such that $j-1$ is a hinge index.

Proposition 6.6. If $j<l$ are two large enough consecutive elements of $J$, then

$$
\frac{c_{l}}{c_{j}} \leq \chi_{j-2}
$$

If $k$ does not belong to $J$, then

$$
\frac{c_{k}}{c_{k-1}}=\chi_{k-1} \text {. }
$$

Proof. Since $k-1$ is not a hinge index, $b_{k-1}$ shares the same end with $b_{k}$ and with $b_{k-2}$ (see Figure 8 , left) so $c_{k} / c_{k-1}=b_{k-1}^{\prime} / b_{k-1}=\chi_{k-1}$.

Since $c_{l}$ is always an edge of $\tau_{l-1}^{\prime}$, we have $c_{l} \leq b_{l-1}^{\prime}$; hence

$$
\frac{c_{l}}{c_{j}} \leq \frac{b_{l-1}^{\prime}}{c_{j}} \leq \frac{b_{j}^{\prime}}{c_{j}}=\frac{c_{j-1}}{b_{j-2}} \leq \frac{b_{j-2}^{\prime}}{b_{j-2}}=\chi_{j-2}
$$

where the equality in the middle just translates the similarity property of the "hinge" triangles $\tau_{j-1}, \tau_{j-1}^{\prime}$ (shaded in Figure 8, right). q.e.d.
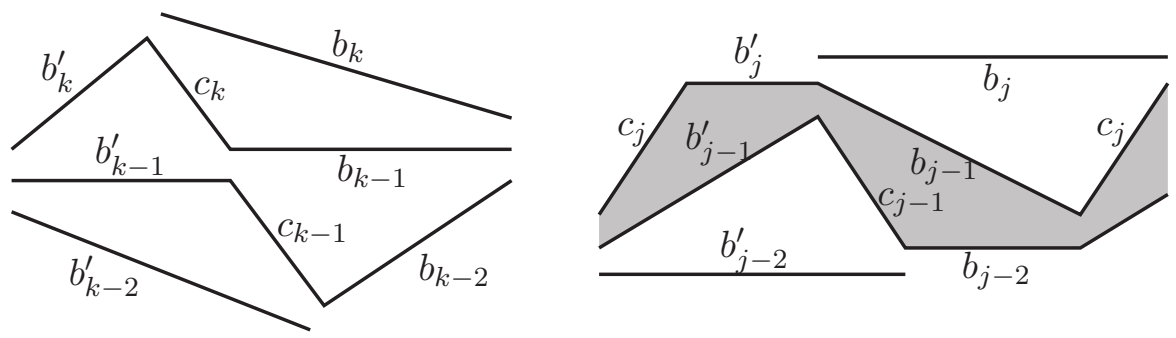

Figure 8. Left: $k-1$ is not a hinge. Right: $j-1$ is a hinge.

\section{Intrinsic convergence of the surfaces $S_{i}$}

7-A. Thickness of the tetrahedra. Consider a tetrahedron $\Delta_{i}$ bounded by the pleated surfaces $S_{i}$ and $S_{i+1}$. Let $Q$ (resp. $Q^{\prime}$ ) be the pleating edge of $S_{i}$ (resp. $S_{i+1}$ ) not lying in $S_{i} \cap S_{i+1}$. Let $s_{i}$ be the shortest segment between $Q$ and $Q^{\prime}$, across $\Delta_{i}$. 
Definition 7.1. Recall the orientations on the edges of $V=\bigcup_{i \in \mathbb{Z}} \Delta_{i}$. Let $\ell_{i}$ be the complex length of the hyperbolic loxodromy along $s_{i}$ sending $Q$ to $Q^{\prime}$, respecting the orientations of $Q, Q^{\prime}$ (with $-\pi<\operatorname{Im} \ell_{i} \leq \pi$ ).

Proposition 7.2. The infinite sum $\sum_{i \in \mathbb{Z}}\left(\ell_{i}\right)$ is absolutely convergent.

Proof. Consider a downward-pointing triangle $\tau$ contributed by $\Delta_{i}$. Label the vertices of $\tau$ by $A, B, C$ in such a way that $A C=b_{i}, B C=b_{i-1}$ and $A B=c_{i}$ (see Figure 6). Let $\gamma_{i}$ be the hyperbolic loxodromy of complex length $\ell_{i}$ along the common perpendicular to $A C$ and $B \infty$, sending $A, C$ to $B, \infty$ (in that order).

Sublemma 7.3. Let $\ell=\ell_{i}=\rho+\theta \sqrt{-1}$ be the complex length of $\gamma_{i}$, with $\theta \in]-\pi, \pi]$. Then $\max \{\rho,|\theta|\} \leq \pi \sqrt{c_{i} / b_{i}}$.

Proof. Up to a plane similarity, we may assume $A=1$ and $C=-1$. Also, for convenience, relabel the edges of $A B C$ by $a, b, c$. Let $L$ be the fixed line of $\gamma_{i}$. The hyperbolic isometry defined by $z \mapsto f(z)=\frac{2 B+1-z}{z+1}$ exchanges the oriented lines $A C$ and $B \infty$, so it reverses the orientation of $L$ around the center of the tetrahedron $A B C \infty$. Therefore, $\gamma_{i}$ is given by $\gamma_{i}(z)=2 B-f(z)$. If $M$ is a matrix of $\gamma_{i}$, one has $\frac{\operatorname{tr}^{2} M}{4 \operatorname{det} M}=\frac{\cosh \ell+1}{2}$. Use $M=\left(\begin{array}{cc}2 B+1 & -1 \\ 1 & 1\end{array}\right)$ to find $B=\cosh \ell$. Compute

$$
\begin{aligned}
\frac{a \pm c}{b} & =\frac{|B+1| \pm|B-1|}{2}=\left|\cosh ^{2} \frac{\ell}{2}\right| \pm\left|\sinh ^{2} \frac{\ell}{2}\right| \\
& =\cosh \frac{\ell}{2} \cosh \frac{\bar{\ell}}{2} \pm \sinh \frac{\ell}{2} \sinh \frac{\bar{\ell}}{2}=\cosh \frac{\ell \pm \bar{\ell}}{2} .
\end{aligned}
$$

Use $\cosh (i \theta)=\cos \theta$ to get $\cosh (\rho)=\frac{a+c}{b}$ and $\cos (\theta)=\frac{a-c}{b}$. The estimates $\operatorname{Arccosh}(y) \leq 2 \sqrt{\frac{y-1}{2}}$ and $\operatorname{Arccos}(y) \leq \pi \sqrt{\frac{1-y}{2}}$, since $\frac{1}{2}\left|\frac{a \pm c}{b}-1\right|$ $\leq \frac{c}{b}$, finally yield $\rho \leq 2 \sqrt{c / b}$ and $|\theta| \leq \pi \sqrt{c / b}$; hence Sublemma 7.3.

q.e.d.

Since $\left(b_{i}\right)$ goes to 2 , Proposition 7.2 will follow if the $c_{i}$ go to 0 fast enough near $+\infty$ (with a similar argument near $-\infty$ ). Such fast decay is given by Proposition 6.6: using the fact that $\left(\chi_{i}\right)$ goes to 0 , we can bound the $c_{i}$ by decreasing geometric sequences on intervals of the form $\llbracket j, l-1 \rrbracket$ where $j<l$ are consecutive elements of the set $J$. q.e.d.

Proposition 7.4. The cusp link has finite width: it is isometric to the Euclidean annulus $\mathbb{S}^{1} \times(0, L)$ for some $L>0$. The ideal triangles whose vertices are $\infty$ and the endpoints of $b_{i}$ accumulate to a certain ideal triangle of $\mathbb{H}^{3}$ as $i$ goes to $+\infty$; the iterates of this ideal triangle under the lift $z \mapsto z+2$ of the hyperelliptic involution are joined side by side and their union contains a vertical Euclidean half-plane (cut off at height 1 above $\mathbb{C})$. 
Proof. The area of a downward-pointing Euclidean triangle defined by the ideal tetrahedron $\Delta_{i}$ is $O\left(c_{i}\right)$ as $i$ goes to $+\infty$, and as in the previous proof, Proposition 6.6 implies that the $\left\{c_{i}\right\}_{i \geq 0}$ have finite sum. Together with Property 6.4, this shows that the downward-pointing triangles accumulate to a segment $b$ in the boundary of the cusp link. The rest of the Proposition follows because $b$ is horizontal, of length 2 . We refer again to Figure 4.

q.e.d.

7-B. Teichmüller charts. Let $\mathcal{T}_{S}$ be the Teichmüller space of the punctured torus: for each $i \in \mathbb{Z}$, we denote by $\mu\left(S_{i}\right) \in \mathcal{T}_{S}$ the intrinsic modulus of the marked surface $S_{i}$ (for the marking $S_{i} \simeq S$ defined before Theorem 1.1). In order to prove that the $\mu\left(S_{i}\right)$ converge in $\mathcal{T}_{S}$, let us first introduce appropriate charts for $\mathcal{T}_{S}$.

Consider a topological ideal triangulation $E$ of the punctured torus $S$, with labeled edges $\epsilon_{1}, \epsilon_{2}, \epsilon_{3}$. Then $E$ defines an isomorphism

$$
h_{E}: \mathbb{P}^{2} \mathbb{R}_{+}^{*}=\left(\mathbb{R}_{+}^{*}\right)^{\left\{\epsilon_{1}, \epsilon_{2}, \epsilon_{3}\right\}} / \mathbb{R}_{+}^{*} \simeq \mathcal{T}_{S} .
$$

Namely, given a hyperbolic metric $g$ on $S$, in order to compute $h_{E}^{-1}(g)$, straighten $E$ to an ideal triangulation for $g$ and return the positive projective triple of Euclidean lengths defined (in the link of the puncture) by the sectors opposite $\epsilon_{1}, \epsilon_{2}, \epsilon_{3}$. We consider the $h_{E}$ as charts of $\mathcal{T}_{S}$. We endow $\mathbb{P}^{2} \mathbb{R}_{+}^{*}$ with the distance $d=d_{\mathbb{P}^{2} \mathbb{R}_{+}^{*}}$ given by

$$
d\left([a: b: c],\left[a^{\prime}: b^{\prime}: c^{\prime}\right]\right):=\min _{\lambda>0} \max \left\{\left|\log \frac{\lambda a}{a^{\prime}}\right|,\left|\log \frac{\lambda b}{b^{\prime}}\right|,\left|\log \frac{\lambda c}{c^{\prime}}\right|\right\} .
$$

It is easy to see that $\left(\mathbb{P}^{2} \mathbb{R}_{+}^{*}, d\right)$ is a complete metric space (isometric, in fact, to the normed vector space $\mathbb{R}^{2}$ whose unit ball is a regular hexagon). Also define

$$
\begin{aligned}
\bar{h}_{E}: \quad \mathbb{P}^{2} \mathbb{C}^{*} & \rightarrow \mathcal{T}_{S} \\
{[a: b: c] } & \mapsto h_{E}([|a|:|b|:|c|]) .
\end{aligned}
$$

In particular, if the pleated punctured torus $S_{i+1}$, pleated along the ideal triangulation $E_{i+1}$, gives rise in the cusp link to a broken (oriented) line whose segments have complex coordinates $(a, b, c)$, then

$$
\mu\left(S_{i+1}\right)=\bar{h}_{E_{i+1}}([a: b: c]) .
$$

Moreover, Figure 9 shows two broken lines corresponding respectively to $S_{i+1}$ and the previous pleated surface $S_{i}$. Since the triangles (links of vertices of $\Delta_{i}$ ) are similar, the complex coordinates of the segments forming the lower broken line are functions of $(a, b, c)$, as shown. Therefore, if we define the substitution formula

$$
\Psi([a: b: c]):=\left(\left[a+b: \frac{b}{a+b} c: \frac{a}{a+b} c\right]\right)
$$




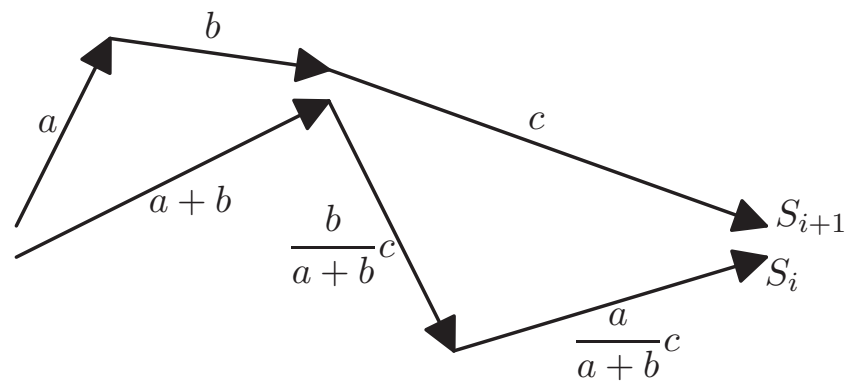

Figure 9. The complex projective triples associated to $S_{i+1}$ and $S_{i}$. These edge orientations do not coincide with those of Figures 5 and 6.

(which is a birational isomorphism from $\mathbb{P}^{2} \mathbb{C}$ to itself), then

$$
\mu\left(S_{i}\right)=\bar{h}_{E_{i}}(\Psi([a: b: c])) .
$$

Finally, by viewing the diagonal move between triangulations $E_{i}$ and $E_{i+1}$ as a flat tetrahedron (as in Figure 1), and a hyperbolic metric as a pleated surface with null pleating, we see that

$$
\mu\left(S_{i+1}\right)=h_{E_{i}}(\Psi([|a|:|b|:|c|])) .
$$

In other words, the restriction $\psi$ of $\Psi$ to $\mathbb{P}^{2} \mathbb{R}_{+}^{*}$ is the chart map $h_{E_{i+1}} \rightarrow$ $h_{E_{i}}$, i.e., the diagram

$$
\begin{array}{lll}
\mathbb{P}^{2} \mathbb{R}_{+}^{*} & \stackrel{\psi}{\psi} & \mathbb{P}^{2} \mathbb{R}_{+}^{*} \\
h_{E_{i}} \searrow & & \swarrow h_{E_{i+1}} \\
& \mathcal{T}_{S} &
\end{array}
$$

commutes. If there are no hinge indices between $i$ and $i+k$ (bounds included), it is easy to check that the chart map $h_{E_{i+k}} \rightarrow h_{E_{i}}$ is, up to a permutation of the coordinates, the map $\psi^{k}$.

Property 7.5. We have $d\left(h_{E_{i}}^{-1} \mu\left(S_{i}\right), h_{E_{i}}^{-1} \mu\left(S_{i+1}\right)\right)=\log \frac{|a|+|b|}{|a+b|}$.

Proof. The right member is

$d\left(\left[|a+b|:\left|\frac{b}{a+b} c\right|:\left|\frac{a}{a+b} c\right|\right],\left[|a|+|b|: \frac{|b|}{|a|+|b|}|c|: \frac{|a|}{|a|+|b|}|c|\right]\right)$.

It is easy to see that $\psi: \mathbb{P}^{2} \mathbb{R}_{+}^{*} \rightarrow \mathbb{P}^{2} \mathbb{R}_{+}^{*}$ is 3 -Lipschitz for $d$. In fact,

Proposition 7.6. There exists $K>0$ such that the $n$-th iterate $\psi^{n}$ is Kn-bilipschitz for all $n>0$. 
Proof. Since

$$
\begin{aligned}
\psi([a: b: c]) & =\left[\frac{(a+b)^{2}}{c}: b: a\right] \quad \text { and } \\
\psi^{-1}([a: b: c]) & =\left[c: b: \frac{(c+b)^{2}}{a}\right]
\end{aligned}
$$

for all $a, b, c>0$, it is enough to check the Lipschitz statement. Define the quantities $A:=\sqrt{a / b} ; C:=\sqrt{c / b}$;

$$
P_{n}:=\frac{A^{n+1}}{C^{n}}+\sum_{i, j \in \mathbb{Z}}\left(\begin{array}{c}
j \\
i-1
\end{array}\right)\left(\begin{array}{c}
n-i \\
j-i
\end{array}\right) A^{2 i-n-1} C^{n-2 j} \text { for all } n \geq-1
$$

(the sum is really on $0<i \leq j \leq n$ ), so that $P_{0}=A, P_{-1}=C, P_{1}=$ $\frac{A^{2}+1}{C}$. We claim that $\psi^{n}[a: b: c]=\left[P_{n}^{2}: 1: P_{n-1}^{2}\right]$ for all $n \geq 0$. The claim is seen by induction on $n$ : the only difficult thing is the induction step $P_{n+1}^{2}=\frac{\left(P_{n}^{2}+1\right)^{2}}{P_{n-1}^{2}}$. First, it is straightforward to check that $P_{n+1}+P_{n-1}=\left(\frac{A}{C}+\frac{C}{A}+\frac{1}{A C}\right) P_{n}$ (using the Pascal relation three times). Hence, for all $n \geq 1$, one has

$$
\begin{aligned}
& \left(P_{n+1} P_{n-1}-P_{n}^{2}\right)-\left(P_{n} P_{n-2}-P_{n-1}^{2}\right) \\
& =P_{n-1}\left(P_{n+1}+P_{n-1}\right)-P_{n}\left(P_{n}+P_{n-2}\right) \\
& =\left(P_{n-1} P_{n}-P_{n} P_{n-1}\right)\left(\frac{A}{C}+\frac{C}{A}+\frac{1}{A C}\right)=0 .
\end{aligned}
$$

Therefore, $P_{n+1} P_{n-1}-P_{n}^{2}=P_{1} P_{-1}-P_{0}^{2}=1$, which proves the induction step. Since $P_{n}$ is a Laurent polynomial in $A, C$, all of whose exponents are in $[-2 n, 2 n]$, and all of whose coefficients are positive, we see that $\log P_{n}$ is $2 n$-bilipschitz in $\log A, \log C$ (hence, in $\log a, \log b, \log c$ ). The Proposition follows.

q.e.d.

The proof of Proposition 7.6 may seem extremely ad hoc and unsatisfactory. However, Proposition 7.6 is a special case of a more general phenomenon for Markoff maps (in the sense of [Bo]), which we describe in [G1]. Further instances of the "Laurent phenomenon", related to the subject of cluster algebras, are described e.g. in $[\mathbf{F Z}, \mathbf{P r}]$.

\section{7-C. Convergence in Teichmüller space.}

Proposition 7.7. The marked hyperbolic surfaces $\left(\mu\left(S_{i}\right)\right)_{i \rightarrow \pm \infty}$ converge in the Teichmüller space $\mathcal{T}_{S}$.

Proof. To prove the $+\infty$-statement, we will fix a large enough index $i$, and prove that the series

$$
\eta_{j}:=d_{\mathbb{P}^{2} \mathbb{R}_{+}^{*}}\left(h_{E_{i}}^{-1} \mu\left(S_{j}\right), h_{E_{i}}^{-1} \mu\left(S_{j+1}\right)\right),
$$

defined for $j>i$, has finite sum (actually $\eta_{j}$ decreases extremely fast).

For $j>i$, consider a downward-pointing triangle $\tau$ contributed by $\Delta_{j}$, with its edge lengths $b_{j}, b_{j-1}$ and $c_{j}$. The angles of $\tau$ at the ends of 
$b_{j}$ are $x_{j}$ and $y_{j}$. By Property 7.5, we have $d\left(h_{E_{j}}^{-1} \mu\left(S_{j}\right), h_{E_{j}}^{-1} \mu\left(S_{j+1}\right)\right)=$ $\log \frac{c_{j}+b_{j-1}}{b_{j}}$. Compute

$$
\begin{aligned}
\log \frac{c_{j}+b_{j-1}}{b_{j}} & \leq \frac{c_{j}+b_{j-1}-b_{j}}{b_{j}} \\
& =\frac{\sin x_{j}+\sin y_{j}-\sin z_{j}}{\sin z_{j}}=\frac{2 \sin \frac{x_{j}}{2} \sin \frac{y_{j}}{2}}{\cos \frac{x_{j}+y_{j}}{2}} \\
& \leq \frac{\sin x_{j} \sin y_{j}}{\sin ^{2} z_{j}} \sin ^{2} z_{j}=\frac{c_{j} b_{j-1}}{b_{j}^{2}} \sin ^{2} w_{j} \leq c_{j} \phi_{j}^{+2}
\end{aligned}
$$

(the inequality at the start of the second line holds for large enough $j$ because $x_{j}+y_{j}=2 w_{j} \rightarrow 0$; for the last inequality, just recall $b_{j} \rightarrow 2$ and $w_{j} \leq \phi_{j}^{+}$). Define $\delta_{j}=c_{j} \phi_{j}^{+2}$, and let $M_{j}$ denote the best bilipschitz constant for the chart map $h_{E_{j}} \rightarrow h_{E_{i}}$. Clearly, $\eta_{j} \leq M_{j} \delta_{j}$.

Let $j<l$ be two consecutive elements of $J$ (see Definition 6.5), and $k \notin J$ an integer. We shall bound the $M_{n} \delta_{n}$ by geometric sequences on intervals of the form $\llbracket j, l-1 \rrbracket$, using Proposition 6.6 as in the proof of Proposition 7.2. Since $\psi$ is 3-bilipschitz, one clearly has $M_{k} \leq 3 M_{k-1}$. By Proposition 6.6,

$$
\frac{M_{k} \delta_{k}}{M_{k-1} \delta_{k-1}} \leq 3 \frac{\delta_{k}}{\delta_{k-1}} \leq 3 \frac{c_{k}}{c_{k-1}}=3 \chi_{k-1}
$$

because $\left(\phi_{k}^{+}\right)$is decreasing. The right member goes to 0 for large $k$.

By Proposition 7.6, $M_{l} \leq(l-j) L \cdot M_{j}$ for some universal $L$, and by Lemma 3.2 (i)-(iii)-(iv), $\phi_{j}^{+}=\phi_{l-1}^{+}+(l-j-1) \phi_{l}^{+} \geq(l-j) \phi_{l}^{+}$. Using Proposition 6.6, it follows that

$$
\frac{M_{l} \delta_{l}}{M_{j} \delta_{j}} \leq(l-j) L \cdot \frac{c_{l}}{c_{j}}\left(\frac{\phi_{l}^{+}}{\phi_{j}^{+}}\right)^{2} \leq \frac{L \chi_{j-2}}{l-j} \leq L \chi_{j-2} .
$$

The right member goes to 0 as $j$ goes to infinity; hence Proposition 7.7.

q.e.d.

\section{Extrinsic convergence of the surfaces $S_{i}$}

Propositions 7.2 and 7.7, together with Corollary 5.3, are the key ingredients to prove that the metric completion of $V=\bigcup_{i \in \mathbb{Z}} \Delta_{i}$ has two boundary components, which are pleated punctured tori with pleating measures $\lambda^{ \pm}$. To this end, we must still understand the limit of the punctured tori $S_{i}$ as embedded objects in $V$, i.e., talk about their extrinsic convergence in the ambient space (as opposed to the intrinsic convergence given by Proposition 7.7). The results in this section should probably be seen as more "technical" than "hard". 
8-A. Pleated surfaces. A pleated surface in a hyperbolic 3-manifold $M$ is by definition a map $\varphi: \Sigma \rightarrow M$ (where $\Sigma$ is a hyperbolic surface) which sends rectifiable arcs to rectifiable arcs of the same length, and such that through each point $p$ of $\Sigma$ runs an open segment $s_{p}$ on which $\varphi$ is totally geodesic. It is known (see [CEG], 5.1.4) that the direction of $s_{p}$ is unique if and only if $p$ belongs to a certain geodesic lamination $\Lambda$ (closed union of disjoint geodesics) in $\Sigma$, and that $\varphi$ is totally geodesic away from $\Lambda$.

To wrestle with pleated surfaces, we will use the fact that if $\varphi$ is a locally convex immersion, and $\Lambda$ has zero Lebesgue measure, then $\Lambda$ comes with a transverse (pleating) measure $\nu_{\Lambda}$. More precisely, $\nu_{\Lambda}$ can be defined on any sufficiently short oriented segment $s$ transverse to $\Lambda$ in the following way (see Sections 7 to 9 of $[\mathbf{B 2}]$ ). First, assume (taking universal covers if necessary) that $\Sigma=\mathbb{H}^{2}$ and $M=\mathbb{H}^{3}$. Immerse $\varphi\left(\mathbb{H}^{2}\right)$ into the Poincaré upper half-space model. Each component of $\mathbb{H}^{2} \backslash \Lambda$ crossed by $s$ can be extended to a subset $A$ of $\mathbb{H}^{2}$ bounded by only (one or) two lines of $\Lambda$ crossed by $s$. Endow $s$ with a transverse orientation. The boundary component of $A$ in $\partial \mathbb{H}^{3}=\mathbb{P}^{1} \mathbb{C}$ on (say) the positive side of the transverse orientation defines an oriented circle arc or line segment $c_{A} \subset \partial \mathbb{H}^{3}=\mathbb{C} \cup\{\infty\}$, of angle $\theta_{A} \in(-2 \pi, 2 \pi)$ (the orientation of $c_{A}$ is induced from $s$, and we may assume $\infty \notin c_{A}$ ). If $c_{A}$ is a line segment or is reduced to a point, we fix $\theta_{A}=0$. The closure of the union of all the $c_{A}$ forms a rectifiable curve $c=c(s) \subset \mathbb{C}$, of length $\sum_{A}$ length $\left(c_{A}\right)$. Then $c$ has a well-defined regular curvature $R C(c)$, defined as the absolutely convergent sum $\sum_{A} \theta_{A}$. But $c$ also has a total curvature $T C(c)$, defined (up to an appropriate multiple of $2 \pi$ ) as the difference between the arguments of the initial and final tangent vectors to $c$. (The appropriate multiple of $2 \pi$ can be determined by closing off the embedded arc $c$ with a broken line, and requiring that the resulting Jordan curve have total curvature $2 \pi)$. Then, $\nu_{\Lambda}(s)$ is defined as the singular curvature $S C(c)=T C(c)-R C(c)$. If $s$ is an arbitrarily long segment of $\mathbb{H}^{2}$ transverse to $\Lambda$, we can define $\nu_{\Lambda}(s)$ by subdividing $s$ into sufficiently short segments $\left(s_{1}, \ldots, s_{n}\right)$ and define $S C(c(s))=\nu_{\Lambda}(s)=\sum_{i=1}^{n} \nu_{\Lambda}\left(s_{i}\right)$.

Conversely, if $\varphi: \mathbb{H}^{2} \rightarrow \mathbb{H}^{3}$ is a pleated immersion and $S C(c(s))$ is well-defined and non-negative for all transverse segments $s$, then $\varphi$ is locally convex with pleating measure $\nu_{\Lambda}$ as above. We refer to [B2] for greater detail.

Finally, the following "equidistributional" notion will be handy.

Definition 8.1. In $\mathbb{R}^{2}$, let $A$ be the union of all lines of equation $x+y=n$ (for $n$ ranging over $\mathbb{Z}$ ); let $B$ be the union of all lines of equation $x=n$; let $C$ be the union of all lines of equation $y=n$. A sequence $\left(X_{1}, \ldots, X_{n}\right)$ taking its values in a set $\{a, b, c\}$ of three elements is called Sturmian if there exists a bijection $\psi:\{a, b, c\} \rightarrow\{A, B, C\}$ 
and a straight segment $\sigma$ of $\mathbb{R}^{2} \backslash \mathbb{Z}^{2}$ such that $\sigma$ first hits $\psi\left(X_{1}\right)$, then $\psi\left(X_{2}\right)$, then $\psi\left(X_{3}\right)$, etc.

Consecutive terms of any Sturmian sequence obviously form a Sturmian sequence. Moreover, it is an easy exercise to show that there exists a universal constant $K^{\text {Sturm }}$ such that if $\left(X_{1}, \ldots, X_{n}\right)$ is Sturmian and $0 \leq k \leq n$, then

$$
\left|\left\{i \leq k \mid X_{i}=a\right\}\right|=\frac{k}{n}\left|\left\{i \leq n \mid X_{i}=a\right\}\right|+\delta \text { where }|\delta| \leq K^{\text {Sturm }} .
$$

8-B. Setup. Define the marked hyperbolic once-punctured torus $S_{+\infty}$, endowed with the hyperbolic metric $\lim _{i \rightarrow+\infty} \mu\left(S_{i}\right)$. There exists a unique compact geodesic lamination $\Lambda_{c}^{+}$of slope $\beta^{+} / \alpha^{+}$on $S_{+\infty}$ (namely, $\Lambda_{c}^{+}$is the topological limit of any sequence of simple closed curves whose slopes converge to $\left.\beta^{+} / \alpha^{+}\right)$. Exactly two lines $\ell, \ell^{\prime}$ issued from the puncture of $S_{+\infty}$ fail to meet $\Lambda_{c}^{+}$: define $\Lambda^{+}=\Lambda_{c}^{+} \sqcup \ell \sqcup \ell^{\prime}$. Then $S_{+\infty}$ is the disjoint union of $\Lambda^{+}$and the interiors of two ideal triangles $A, A^{\prime}$. The union $A \cup A^{\prime} \cup \ell \cup \ell^{\prime}$ is a punctured ideal bigon.

Recall the ideal tetrahedra $\Delta_{i}$, the space $V=\bigcup_{i \in \mathbb{Z}} \Delta_{i}$ and the hyperelliptic involution $\iota: V \rightarrow V$ which reverses all edge orientations. The slopes of the (oriented) pleating lines of the surface $S_{i}$ (between $\Delta_{i-1}$ and $\left.\Delta_{i}\right)$ are elements of $\mathbb{P}^{1} \mathbb{Q}$ projecting to $0,1, \infty$ in $\mathbb{P}^{1}(\mathbb{Z} / 2 \mathbb{Z})$ : accordingly, we call these pleating lines $l_{i}^{0}, l_{i}^{1}, l_{i}^{\infty}$. For $* \in\{0,1, \infty\}$, denote by $\omega_{i}^{*}$ the unique point of $l_{i}^{*}$ fixed under the hyperelliptic involution (the $\omega_{i}^{*}$ are called Weierstrass points). Let $s_{i}^{*}$ be the segment from $\omega_{i}^{*}$ to $\omega_{i+1}^{*}$ (across the tetrahedron $\Delta_{i}$ if $\left.\omega_{i}^{*} \neq \omega_{i+1}^{*}\right)$ : each $s_{i}^{*}$ is contained in a (pointwise) fixed line $\Omega^{*}$ of the hyperelliptic involution.

Fix the value of the superscript $*$ (soon we shall omit it). Fix a point $\omega$ in $\mathbb{H}^{2}$ and an oriented line $l$ through $\omega$. For each (oriented) surface $S_{i}$, consider the oriented, marked universal covering $\pi_{i}:\left(\mathbb{H}^{2}, \omega, l\right) \rightarrow$ $\left(S_{i}, \omega_{i}^{*}, l_{i}^{*}\right)=\left(S_{i}, \omega_{i}, l_{i}\right)$. Endow a universal cover $\widetilde{V}$ of $V$ with lifts $\widetilde{\omega}_{i}$ of the $\omega_{i}$ connected by lifts of the segments $s_{i}^{*}=s_{i}$, and fix a developing map $\Phi: \widetilde{V} \rightarrow \mathbb{H}^{3}$. There is a unique map $h_{i}$ such that the diagram

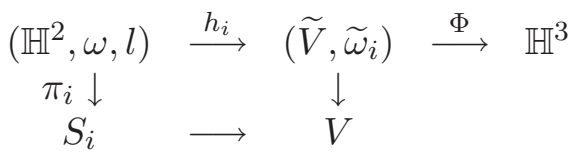

commutes. We will prove that the (developing) pleated immersions

$$
\varphi_{i}=\Phi \circ h_{i}: \mathbb{H}^{2} \rightarrow \mathbb{H}^{3}
$$

converge as pleated maps to a pleated immersion $\varphi_{+\infty}$.

8-C. Convergence of the $\varphi_{i}$. By Proposition 7.2, since the segments $s_{i}^{*}=s_{i}$ all belong to the same fixed line of the hyperelliptic involution, it is already clear that the restriction of $\varphi_{i}$ to $l$ converges to a totally geodesic embedding of the line $l$ into $\mathbb{H}^{3}$ (the convergence is uniform on 
all compacta of $l$ ). By Ascoli's theorem, since the $\varphi_{i}$ are 1-Lipschitz, there exists an increasing sequence $\nu$ such that the $\varphi_{\nu(i)}$ converge to a certain map $\varphi_{+\infty}$, uniformly on all compact sets of $\mathbb{H}^{2}$. In Section 8-E below, $\varphi_{+\infty}$ is shown to be independent of the subsequence $\nu$ : in anticipation, we now abusively write $\varphi_{i}$ instead of $\varphi_{\nu(i)}$.

We shall work in the projective tangent bundle $\mathcal{E}=\mathbb{P} T \mathbb{H}^{2}$, a circle bundle over $\mathbb{H}^{2}$ in which geodesic laminations naturally live as closed sets. Length and angle measurements define a (canonical) complete Riemannian metric on $\mathcal{E}$. For $K \subset \mathbb{H}^{2}$ compact and $A, B \subset \mathcal{E}$ closed, let $K^{\mathcal{E}} \subset \mathcal{E}$ be the preimage of $K$ under the natural projection $\mathcal{E} \rightarrow \mathbb{H}^{2}$, and define

$$
d_{K}(A, B):=\min \left\{\delta>0 \mid A \cap K^{\mathcal{E}} \subset \mathcal{N}_{\delta}(B), B \cap K^{\mathcal{E}} \subset \mathcal{N}_{\delta}(A)\right\}
$$

where $\mathcal{N}_{\delta}(X)$ denotes the set of points within $\delta$ of $X$. Then $\inf \left(1, d_{K}\right)$ is a pseudometric, and the set of closed subsets of $\mathcal{E}$ is compact for the Hausdorff metric

$$
d_{H}(A, B)=\sum_{n>0} 2^{-n} \min \left(1, d_{K_{n}}(A, B)\right)
$$

where the $K_{n}$ are concentric balls of radius $n$.

Observe that $S_{+\infty}$ also has Weierstrass points $\omega_{+\infty}^{*}$, belonging to leaves $l_{+\infty}^{*}$ of the geodesic lamination $\Lambda^{+}$. Fixing the value of $*$ as before, denote by $\pi_{+\infty}:\left(\mathbb{H}^{2}, \omega, l\right) \rightarrow\left(S_{+\infty}, \omega_{+\infty}^{*}, l_{+\infty}^{*}\right)$ an oriented universal cover.

A consequence of Proposition 7.7 is that the lifts to $\mathcal{E}$ of the $\pi_{i}^{-1}\left(l_{i}^{0} \cup\right.$ $\left.l_{i}^{1} \cup l_{i}^{\infty}\right)$ converge for $d_{H}$ to the lift of $\pi_{+\infty}^{-1}\left(\Lambda^{+}\right)$. We know that $U=$ $\pi_{+\infty}^{-1}\left(S_{+\infty} \backslash \Lambda^{+}\right) \subset \mathbb{H}^{2}$ is a disjoint union of (open) ideal triangles, of full Lebesgue measure in $\mathbb{H}^{2}$. For any connected compact set $K \subset U$, we have $K \cap \pi_{i}^{-1}\left(l_{i}^{0} \cup l_{i}^{1} \cup l_{i}^{\infty}\right)=\emptyset$ for $i$ large enough, so $\varphi_{+\infty}$ is totally geodesic on $K$. Therefore, $\varphi_{+\infty}$ is totally geodesic on each component of $U$. Since $\varphi_{+\infty}$ is clearly 1-Lipschitz, we can approximate any segment in the geodesic lamination $\mathbb{H}^{2} \backslash U$ by segments in $U$ to show that $\varphi_{+\infty}$ is totally geodesic on each leaf of $\mathbb{H}^{2} \backslash U$. By Lemma 5.2.8 in [CEG], $\varphi_{+\infty}$ sends rectifiable segments to rectifiable segments of the same length, and is a pleated map.

Note finally that equivariance of $\varphi_{+\infty}$ comes for free: if $\Gamma$ is the fundamental group of the punctured torus (or equivalently, of $V$ ), consider the representation $\rho: \Gamma \rightarrow \mathrm{Isom}^{+}\left(\mathbb{H}^{3}\right)$ given by the developing map $\Phi$ from (18), and the representations $\rho_{n}: \Gamma \rightarrow \operatorname{Isom}^{+}\left(\mathbb{H}^{2}\right)$ which satisfy $\varphi_{n} \circ \rho_{n}(g)=\rho(g) \circ \varphi_{n}$ for all $g \in \Gamma$. By Proposition 7.7, the $\rho_{n}$ converge to some $\rho_{+\infty}$. Convergence of the $\varphi_{n}$ immediately implies $\varphi_{+\infty} \circ \rho_{+\infty}(g)=\rho(g) \circ \varphi_{+\infty}$.

8-D. The map $\varphi_{+\infty}$ is a topological immersion. Define $\mathcal{P}:=$ $\pi_{+\infty}^{-1}\left(\Lambda^{+}\right) \subset \mathbb{H}^{2}$, which contains the pleating locus of $\varphi_{+\infty}$. To prove 
$\varphi_{+\infty}$ is an immersion, it is enough to find a short geodesic segment $m$ of $\mathbb{H}^{2}$, through the base point $\omega$, transverse to $\mathcal{P}$, and prove that $\varphi_{+\infty}$ is an immersion on the union $\Upsilon$ of all strata (lines and complementary ideal triangles) of $\mathcal{P}$ crossed by $m$ (indeed, $\pi_{+\infty}(\Upsilon)$ is all of $S_{+\infty}$, by density of each leaf of $\Lambda^{+}$). Clearly, $\varphi_{+\infty}$ is already an immersion near any point of $\mathbb{H}^{2} \backslash \mathcal{P}$. To deal with the remaining points, we give below an argument whose main ingredients are of three different natures:

- Existence of "Sturmian" bounds on the algebraic sum of pleating angles met by a short arc $m$ transverse to the pleating lamination of $S_{i}$ (this is the key fact that prevents $\varphi_{i}$ from "wrapping" too much).

- Classical facts about the upper half space model: first, the hyperbolic metric near height 1 and the "ambient" Euclidean metric are controlled in terms of each other. Second, if a hyperbolic line stays close to the line from -1 to 1 in a large neighborhood of the point above the origin, then its endpoints are close to -1 and 1 .

- A priori properties of the maps $\varphi_{i}$ : they are 1-Lipschitz; their pleating loci converge in the Hausdorff topology; and the injectivity radius for the intrinsic metric of $S_{i}$ near a Weierstrass point is bounded away from 0 independently of $i$ (these last two facts follow from Proposition 7.7).

Choose a small $\mu_{1}>0$, and pick $k \in \mathbb{Z}$ large enough so that $\phi_{k}^{+} \leq \mu_{1}$. Let $q$ be the rational opposite the Farey edge $e_{k}$, on the same side as $\beta^{-} / \alpha^{-}$, so that $\lambda^{+}(q)=\phi_{k}^{+}$. Let $m_{i}^{\mathcal{C}}$ be the simple closed geodesic of slope $q$ in $S_{i}$ (equipped with the intrinsic metric): for some superscript $*$ independent of $i$, the Weierstrass point $\omega_{i}=\omega_{i}^{*}$ belongs to $m_{i}^{\mathcal{C}}$. By Proposition 7.7 and Hausdorff convergence, there exist $\mu_{2}, \mu_{3}>0$ such that for all $i>k$, the angle between $m_{i}^{\mathcal{C}}$ and the pleating line $l_{i}$ of $S_{i}$ at $\omega_{i}$ is at least $\mu_{2}$, and the segment $m_{i}$ of $m_{i}^{\mathcal{C}}$ of length $2 \mu_{3}$, centered at $\omega_{i}$, is embedded in $S_{i}$. The ends of $m_{i}$ are at distance at least $\frac{1}{2} \mu_{2} \mu_{3}$ from the pleating line $l_{i}$ in $S_{i}$. Observe that the simple closed curve $m_{i}^{\mathcal{C}}$ meets the pleating edges of $S_{i}$ in a Sturmian order: therefore, by (17), the algebraic sum of the pleating angles crossed by any given subsegment of $m_{i}$ does not exceed $2 \lambda_{i}(q)$ in absolute value; we have $2 \lambda_{i}(q) \leq 2 \lambda^{+}(q) \leq 2 \mu_{1}$. Finally, let $\kappa_{i}$ be a subsegment of the pleating line $l_{i}$, centered at $\omega_{i}$, of length 2 : if $\mu_{3}$ is small enough, any pleating line $L$ met by $m_{i}$ makes an angle at least $\frac{1}{2} \mu_{2}$ with $m_{i}$, and (being disjoint from, but very close to $l_{i}$ ) comes within $3 \mu_{3}$ of both ends of $\kappa_{i}$ for the intrinsic metric of $S_{i}$.

We proceed following Section 8-A. Arrange the developing map $\Phi$ : $\widetilde{V} \rightarrow \mathbb{H}^{3}$ in the upper half-space model so that the $\left\{\varphi_{i}(\omega)\right\}_{i \in \mathbb{Z}}$ lie on the line $0 \infty$ at heights less than 1 , and $\varphi_{+\infty}(l)$ is the oriented line from -1 to 1 . Consider lifts $\widetilde{m}_{i}$ of the arcs $m_{i}$ through the $\varphi_{i}(\omega)$. By the above 
(considering lifts of the $\kappa_{i}$ ), if $\mu_{3}$ is small enough, any pleating line of $\varphi_{i}\left(\mathbb{H}^{2}\right)$ met by $\widetilde{m}_{i}$ has its endpoints within distance $1 / 2$ from 1 and -1 in $\mathbb{C}$ (recall $\varphi_{i}$ is 1-Lipschitz, and the lifts of the segments $\kappa_{i}$ converge to a subsegment of $\left.\varphi_{+\infty}(l)\right)$. Following Subsection 8-A, let $c_{i}^{+1}$ (resp. $\left.c_{i}^{-1}\right)$ denote the piecewise smooth curve in $\mathbb{C}$ defined by the transverse segment $\widetilde{m}_{i}$ of $\varphi_{i}\left(\mathbb{H}^{2}\right)$ near 1 (resp. -1 ).

Let $\sigma \subset \mathbb{H}^{3}$ be a subsegment of $\widetilde{m}_{i}$ across an ideal triangle of $\varphi_{i}\left(\mathbb{H}^{2}\right)$. Let $\sigma^{\prime} \subset \mathbb{C}$ be the circle arc (not reduced to a point) contributed by $\sigma$ to $c_{i}^{ \pm 1}$. By the above, for some universal $K_{1}>0$,

$$
\frac{\mu_{2}}{K_{1}} \leq \frac{\text { euclidean length of } \sigma^{\prime}}{\text { hyperbolic length of } \sigma} \leq K_{1} \text {. }
$$

(This $K_{1}$ is universal in the sense that it is independent of $i, \mu_{1}, \mu_{2}, \mu_{3}$ provided the $\mu$ 's are small enough and $i$ is large enough.) In particular, the $c_{i}^{ \pm 1}$ have length at most $2 K_{1} \mu_{3}$. But the regular curvature radii of $c_{i}^{ \pm 1}$ are at least $\frac{1}{2}$ (the corresponding circles come near 1 and -1 ), so the total regular curvature of $c_{i}^{ \pm 1}$ is at most $4 K_{1} \mu_{3}$. As we already observed (using Sturmian bounds), the total singular curvature on any subinterval of $c_{i}^{ \pm 1}$ is at most $2 \mu_{1}$. If the $\mu$ 's are small enough, it follows that all tangent vectors of $c_{i}^{ \pm 1}$ have complex arguments within $[\pi / 3,2 \pi / 3]$ (or indeed, any fixed closed neighborhood of $\pi / 2$ ). As a consequence, if $\tau$ ranges over the ideal triangles of $\varphi_{i}\left(\mathbb{H}^{2}\right)$ crossed by $\widetilde{m}_{i}$, and $p: \mathbb{H}^{3} \rightarrow \mathbb{C}$ is the vertical projection, the different $p(\tau)$ intersect only along their edges: so $\varphi_{i}\left(\bigcup_{\tau} \tau\right)$ is an embedded surface (which we can see, say, as the graph of a function from an open set of $\mathbb{C}$ to $\left.\mathbb{R}^{+}\right)$. Moreover, define the thickness of $p(\tau)$ as the length of the segment $p(\tau) \cap \sqrt{-1} \mathbb{R}$ (of the imaginary axis). Then, for some universal $K_{2}>0$,

$$
\frac{\text { thickness of } p(\tau)}{\text { hyperbolic length of } \tau \cap \widetilde{m}_{i}} \geq \frac{\mu_{2}}{K_{2}}
$$

because $\tau$ has its (ideal) vertices near 1 and -1 , while the hyperbolic plane carrying $\tau$ is roughly orthogonal to the line $0 \infty$.

To conclude concerning $\varphi_{+\infty}$, define a lift $m^{\mathcal{C}}$ through $\omega \in \mathbb{H}^{2}$ of the simple closed geodesic of slope $q$ in $S_{+\infty}$, and a subsegment $m$ of $m^{\mathcal{C}}$, of length $2 \mu_{3}$, centered at $\omega$. The angle between $m$ and any pleating line of $\Lambda^{+}$it encounters is at least $\frac{1}{2} \mu_{2}$. Let $I$ be the (infinite) collection of ideal triangles of $\pi_{+\infty}^{-1}\left(S_{+\infty} \backslash \Lambda^{+}\right)$crossed by $m$. For $\tau \in I$, denote by $|\tau|$ the hyperbolic length of $m \cap \tau$. By convergence in the Hausdorff metric, $\varphi_{+\infty}(\tau)$ is approached by triangles of the $\varphi_{i}\left(\mathbb{H}^{2}\right)$, of thickness at least $\frac{|\tau| \mu_{2}}{2 K_{2}}$ : so $p\left(\varphi_{+\infty}(\tau)\right)$ has nonzero thickness. Injectivity on the closure $\Upsilon$ of $\bigcup_{\tau \in I} \tau$ follows: if $x, x^{\prime} \in \Upsilon$ do not belong to the same stratum of the lamination $\mathcal{P}=\pi_{+\infty}^{-1}\left(\Lambda^{+}\right)$, find $\tau$ separating $x$ from $x^{\prime}$ to prove that $p \circ \varphi_{+\infty}(x) \neq p \circ \varphi_{+\infty}\left(x^{\prime}\right)$. Thus $p \circ \varphi_{+\infty}$ defines an embedding of $\Upsilon$ into 
$\mathbb{C}$, and since $\pi_{+\infty}(\Upsilon)$ is all of $S_{+\infty}$, the equivariant surface $\varphi_{+\infty}\left(\mathbb{H}^{2}\right)$ is topologically immersed in $\mathbb{H}^{3}$.

8-E. Pleating measure of $\varphi_{+\infty}$. These arguments can be extended to prove that the pleating measure of $\varphi_{+\infty}$, as defined in 8 -A, is the limit of the pleating measures of the $\varphi_{i}$ : the rectifiable curves $c_{+\infty}^{ \pm 1} \subset \mathbb{C}$ defined near \pm 1 by $\varphi_{+\infty}$ have lengths $\ell^{ \pm 1}$, and for any $\varepsilon>0$, there exists a finite disjoint union of circle $\operatorname{arcs} \gamma_{\iota}$ in $c_{+\infty}^{ \pm 1}$ whose lengths add up to at least $\ell^{ \pm 1}-\varepsilon$ (moreover the tangent direction of $c_{+\infty}^{ \pm 1}$, like that of $c_{i}^{ \pm 1}$, is everywhere within $\pi / 6$ of the vertical axis). The $\gamma_{\iota}$ can be approached by (unions of) arcs of the $c_{i}^{ \pm 1}$, and the regular curvature not contributed by the $\gamma_{\iota}$ is bounded by $3 \varepsilon$ (because all curvature radii are at least $\frac{1}{2}$ ). Letting $\varepsilon$ go to 0 , this shows that the total curvature and regular curvature of the $c_{i}^{ \pm 1}$ converge to those of $c_{+\infty}^{ \pm 1}$. It follows that the pleating (or singular curvature) of $\varphi_{+\infty}$ is the limit of the pleatings of the $\varphi_{i}$ (on any transverse arc, and therefore, on any simple closed curve): that pleating is simply (a lift of) $\lambda^{+}$, by Corollary 5.3. By Theorem D of [B2], $\varphi_{+\infty}$ is completely determined by its pleating $\lambda^{+}$and the intrinsic metric of $S_{+\infty}$. In particular, $\varphi_{+\infty}=\lim \varphi_{\nu(i)}$ is independent of the original subsequence $\nu$, and the $\varphi_{i}$ converge to an equivariant pleated map whose pleating is given by $\lambda^{ \pm}$, as $i$ goes to $\pm \infty$.

8-F. Completeness. The construction of 8-D further allows us to embed the universal cover $\widetilde{V}$ of $V=\bigcup_{i \in \mathbb{Z}} \Delta_{i}$ into a (topological) manifold with boundary $\widetilde{V}_{\partial}$, as follows. For each $x \in \mathbb{H}^{2}$, consider a neighborhood $U_{x}$ of $x$ such that $\varphi_{+\infty}$ is an embedding on $U_{x}$ (for $x$ in the set $\Upsilon$, such $U_{x}$ are given by 8 - $\mathrm{D}$; in general we use equivariance). Then $\varphi_{+\infty}\left(U_{x}\right)$, which has a well-defined transverse ("outward") orientation, splits a small ball $B_{x}$ centered at $\varphi_{+\infty}(x)$ into two (topological) hemispheres, which we can call "inner" and "outer", referring to the transverse orientation. The inner hemispheres $H_{x}$, for $x$ ranging over $\mathbb{H}^{2}$, can be patched together to obtain a manifold with boundary $H$. Without loss of generality, the balls $B_{x}$ can be chosen small enough so that, by the construction of Section 8-D, each $H_{x} \backslash \varphi_{+\infty}\left(U_{x}\right)$ is identified with a subset of $\widetilde{V}$, embedded in $\mathbb{H}^{3}$. Then, $H$ can further be patched to $\widetilde{V}$. Since each $H_{x}$ is homeomorphic to $\mathbb{R}^{2} \times \mathbb{R}^{+}$, the space $\widetilde{V}_{\partial}=\widetilde{V} \cup H$ is a possibly non-complete (but locally compact) topological manifold with boundary.

Proposition 8.2. The action of the fundamental group $\Gamma$ of the punctured torus $S$ on $\widetilde{V}$ extends to a properly discontinuous action on $\widetilde{V}_{\partial}$.

Proof. Provided that the hemispheres $H_{x}$ are chosen in an equivariant fashion, the action of $\Gamma$ on $\widetilde{V}_{\partial}$ is well-defined. The action is already 
properly discontinuous at every point $x$ of $\widetilde{V}$ (namely, as $g$ ranges over $\Gamma$, the $g x$ do not accumulate at $x$ ). But if $\Gamma$ acts without fixed points and by isometries on a locally compact metric space $X$, the set of $x \in X$ such that the action is properly discontinuous at $x$ is open (obviously) as well as its complement: if $g_{n} x \rightarrow x$ for some sequence $\left(g_{n}\right)$ of $\Gamma \backslash\{1\}$ and $U$ is a compact neighborhood of $x$, then $U$ contains a ball of radius $\varepsilon$ centered at $x$, and whenever $d\left(x, x^{\prime}\right) \leq \varepsilon / 2$, the $g_{n} x^{\prime}$ accumulate at some point of $U$, so the action is not totally discontinuous at $x^{\prime}$. The Proposition follows by connectedness of $\widetilde{V}_{\partial}$.

q.e.d.

As a consequence, the space $V_{\partial}:=\widetilde{V}_{\partial} / \Gamma$ is a (topological) manifold with boundary, containing $V=\bigcup_{i \in \mathbb{Z}} \Delta_{i}$; and $\partial V_{\partial}$ consists of two pleated punctured tori (intrinsically isometric to $S_{+\infty}$ and another surface $S_{-\infty}$ ) with pleatings $\lambda^{+}$and $\lambda^{-}$.

Proposition 8.3. The manifold with boundary $V_{\partial}$ is complete.

Proof. Consider the metric completion $\bar{V} \supset V_{\partial}$ and assume the inclusion is strict. Define a continuous function $f: V_{\partial} \rightarrow \mathbb{R}^{>0}$ by $f(x)=$ $d\left(x, \bar{V} \backslash V_{\partial}\right)$. By assumption, $\inf (f)=0$.

Consider the immersion of $\widetilde{V}_{\partial}$ into the upper half-space model of $\mathbb{H}^{3}$ obtained by sending a lift of the cusp to infinity (Figures 4 and 5-6). The image of $\partial \widetilde{V}_{\partial}$ contains, in particular, vertical half-planes (interrupted at some height above $\mathbb{C}$ ) by Proposition 7.4. Therefore, any geodesic of $\widetilde{V}_{\partial}$ starting high enough above $\mathbb{C}$ is defined for all times $t \leq 1$ (unless it hits $\left.\partial \widetilde{V}_{\partial}\right)$. As a result, if $H \subset V_{\partial}$ denotes a small enough open horoball neighborhood of the cusp, we have $f \geq 1$ on $H$.

For $i \in \mathbb{Z}$, consider the compact set $K_{i}:=S_{i} \backslash H$ in $V_{\partial}$. There exists a ball $B$ of $\mathbb{H}^{2}$ centered at the base point $\omega$, with radius independent of $i$, such that $K_{i} \subset \pi_{i}(B)$ : by convergence of $\left(\varphi_{i}\right)$, the $\pi_{i}(B)$ converge metrically to a compact subset $K^{\prime}$ of $\partial V_{\partial}$, on which $f$ is positive. Therefore, $f$ is bounded away from 0 on some neighborhood $U$ of $K^{\prime}$ in $V_{\partial}$, and $K_{i} \subset U$ for large enough $i$. So $f$ is bounded away from 0 on $\bigcup_{i \in \mathbb{Z}} K_{i}$, and therefore on $\bigcup_{i \in \mathbb{Z}} S_{i}$.

However, assume $\gamma(t)$ is a rectifiable 1-Lipschitz arc of $V_{\partial}$, defined for $t \in(0, M]$, with no limit at 0 . For any $\varepsilon>0$, the restriction $\gamma_{\mid(0, \varepsilon]}$ meets $V_{\partial} \backslash \partial V_{\partial}$ (because $\partial V_{\partial}=S_{+\infty} \sqcup S_{-\infty}$ is complete), but then $\gamma_{\mid(0, \varepsilon]}$ must meet $\Delta_{i}$ for an unbounded set of indices $i$ (any finite union of tetrahedra is complete). Therefore, we can find a positive sequence $t_{n} \rightarrow 0$ such that $\gamma\left(t_{n}\right) \in \bigcup_{i \in \mathbb{Z}} S_{i}$. Clearly, $f\left(\gamma\left(t_{n}\right)\right) \leq t_{n}$ which goes to 0 : a contradiction. So $V_{\partial}$ is completer q.e.d.

8-G. A quasifuchsian punctured-torus group. The end of the argument is now quite standard: recall the complete manifold with locally 
convex boundary $\widetilde{V}_{\partial}$, which is a universal cover of $\bar{V}=V_{\partial}$. Given distinct points $x, x^{\prime} \in \widetilde{V}_{\partial}$, consider a shortest possible path $\gamma$ from $x$ to $x^{\prime}$. If $\gamma$ has an interior point in $\partial \widetilde{V}_{\partial}$, by local convexity, we must have $\gamma \subset \partial \widetilde{V}_{\partial}$, and $\gamma$ is a geodesic segment of $\partial \widetilde{V}_{\partial}$. If not, $\gamma$ is (the closure of) a geodesic segment of $\widetilde{V}$. At any rate, the extended developing map $\Phi: \widetilde{V}_{\partial} \rightarrow \mathbb{H}^{3}$ is an embedding (it sends $\gamma$ to a segment with distinct endpoints) and has a closed convex image $C$, endowed with a properly discontinuous action of the fundamental group $\Gamma$ of $S$ (Proposition 8.2). The action extends properly discontinuously to $\mathbb{H}^{3}$ (this can be seen by projecting any point of $\mathbb{H}^{3}$ to $C$ ). The manifold $\mathbb{H}^{3} / \Gamma$ contains $\bar{V} \simeq C / \Gamma$, which has the desired boundary pleatings $\lambda^{ \pm}$. Clearly, $C$ is the smallest closed, convex set containing all parabolic fixed points of $\Gamma$ (which are the ideal vertices of all lifts of the tetrahedra $\Delta_{i}$ ); therefore, $\bar{V}$ is the convex core, and $\Gamma$ is quasifuchsian, with pleating data $\lambda^{ \pm}$. Theorem 1.1 is proved.

\section{Application: the EPH theorem}

Let us quickly recall the correspondence between the horoballs of $\mathbb{H}^{3}$ and the vectors in the positive isotropic (light) cone of Minkowski space $\mathbb{R}^{3+1}$. Endow $\mathbb{R}^{4}$ with the Lorentzian product $\left\langle(x, y, z, t) \mid\left(x^{\prime}, y^{\prime}, z^{\prime}, t^{\prime}\right)\right\rangle$ $:=x x^{\prime}+y y^{\prime}+z z^{\prime}-t t^{\prime}$. Define

$$
X:=\left\{v=(x, y, z, t) \in \mathbb{R}^{4} \mid t>0 \text { and }\langle v \mid v\rangle=-1\right\} .
$$

Then $\langle$.$| .| \rangle$ restricts to a Riemannian metric on $X$ and there is an isometry $X \simeq \mathbb{H}^{3}$, with $\operatorname{Isom}^{+}(X)$ a component of $S O_{3,1}(\mathbb{R})$. This isometry takes hyperbolic planes in $\mathbb{H}^{3}$ to intersections of $X$ with (linear) hyperplanes. We will identify the point $(x, y, z, t)$ of $X$ with the point at Euclidean height $\frac{1}{t+z}$ above the complex number $\frac{x+i y}{t+z}$ in the Poincaré upper half-space model. Under this convention, the closed horoball $H_{d, \zeta}$ of Euclidean diameter $d$ centered at $\zeta=\xi+i \eta \in \mathbb{C}$ in the upper halfspace model is identified with the set $\left\{v \in X \mid\left\langle v \mid v_{d, \zeta}\right\rangle \geq-1\right\}$, where

$$
v_{d, \zeta}=\frac{1}{d}\left(2 \xi, 2 \eta, 1-|\zeta|^{2}, 1+|\zeta|^{2}\right)
$$

We therefore identify $H_{d, \zeta}$ with the point $v_{d, \zeta}$ of the light cone. Similarly, the closed horoball $H_{h, \infty}$ of points at Euclidean height no less than $h$ in the half-space model corresponds to $\left\{v \in X \mid\left\langle v \mid v_{h, \infty}\right\rangle \geq-1\right\}$ where

$$
v_{h, \infty}=(0,0,-h, h),
$$

so we identify $H_{h, \infty}$ with $v_{h, \infty}$.

Consider the following objects: a complete oriented hyperbolic 3manifold $M$ with one cusp, a horoball neighborhood $H$ of the cusp, a universal covering $\pi: \mathbb{H}^{3} \rightarrow M$, and the group $\Gamma \subset \operatorname{Isom}^{+}\left(\mathbb{H}^{3}\right)$ of deck transformations of $\pi$. Then $H$ lifts to a family of horoballs 
$\left(H_{i}\right)_{i \in \mathcal{I}}$ in $\mathbb{H}^{3}$, corresponding to a family of isotropic vectors $\left(v_{i}\right)_{i \in \mathcal{I}}$ in Minkowski space. The closed (linear) convex hull $C$ of $\left\{v_{i}\right\}_{i \in \mathcal{I}}$ is $\Gamma$ invariant, and its boundary $\partial C$ comes with a natural decomposition $\widetilde{\mathcal{D}}$ into polyhedral facets. Let $V$ be the interior of the convex core of $M$. In [AS], Akiyoshi and Sakuma extended the Epstein-Penner convex hull construction to prove that $\widetilde{\mathcal{D}}$ defines an $H$-independent decomposition $\mathcal{D}^{\text {Geom }}$ of $V$ into ideal hyperbolic polyhedra, typically tetrahedra, allowing for a few clearly defined types of degeneracies. In [ASWY2], with Wada and Yamashita, they also conjectured

Theorem 9.1. If $M$ is quasifuchsian, homeomorphic to the product of the punctured torus with the real line, and has irrational pleating laminations of slopes $\beta^{+} / \alpha^{+}$and $\beta^{-} / \alpha^{-}$, the decomposition $\mathcal{D}^{\text {Geom }}$ of $V$ is combinatorially the triangulation $\left\{\Delta_{i}\right\}_{i \in \mathbb{Z}}$ defined in Section 1.

Proof. It is known (see [Se] or Corollary 10.8 below) that quasifuchsian punctured-torus groups are fully determined by their measured pleating laminations. It follows that the manifold constructed in Sections $1-8$, with the same pleating data as $M$, is isometric to $M$. Recall the triangulated space $\widetilde{V} \subset \mathbb{H}^{3}$ of (18), which is a universal cover of the union $\bigcup_{i \in \mathbb{Z}} \Delta_{i}$ of all tetrahedra $\Delta_{i}$. Given a horoball neighborhood $H$ of the cusp of $M$, consider its lifts $\left(H_{i}\right)_{i \in \mathcal{I}}$ in $\mathbb{H}^{3}$ and the corresponding isotropic vectors $\left(v_{i}\right)_{i \in \mathcal{I}}$ in Minkowski space $\mathbb{R}^{4}$. Then each tetrahedron $\Delta$ of $\widetilde{V}$ has its vertices at the centers of four horoballs $H_{1}, \ldots, H_{4}$ : we introduce the convex hull $\tau_{\Delta}$ of $v_{1}, \ldots, v_{4}$, and define $D:=\bigcup_{\Delta} \tau_{\Delta}$. The central projection to the hyperboloid $X$ with respect to the origin sends $\tau_{\Delta}$ homeomorphically to the lift of $\Delta$ in $X$, so the interiors of the $\tau_{\Delta}$ are pairwise disjoint, and each $\tau_{\Delta}$ comes with a transverse "upward" orientation $\vec{u}$ (given by any vector of $\tau_{\Delta}$, i.e., with head in $\tau_{\Delta}$ and foot at the origin). The theorem claims exactly that $D \subset \partial C$ as polyhedral complexes (the inclusion is expected to be strict, since $\partial C$ also contains faces projecting, say, to the boundary of the convex core - they are analyzed in detail in $[\mathbf{A S}]$ ).

Suppose the codimension- 1 complex $D \subset \mathbb{R}^{4}$ is locally convex with $\vec{u}$ pointing inward and nonzero bending angles. We can then prove $D \subset \partial C$ : for any subset $Y$ of a real vector space, define $I Y:=\{t y \mid t \geq$ $1, y \in Y\}$. Since the interior of the convex core is convex, $I D$ is a convex set (for any non-collinear $x, y \subset I D$, the 2-dimensional quadrant $P=\mathbb{R}^{+} x+\mathbb{R}^{+} y$ intersects the hyperboloid $X$ along a hyperbolic segment of the universal cover $\widetilde{V}$ of the convex core, and $P \cap I D$ is convex by local convexity of $D$ : so the segment $[x, y]$ is in $I D)$. Moreover, $I D$ contains all the isotropic vectors $\left\{v_{i}\right\}_{i \in \mathcal{I}}$, so its closure $\overline{I D}$ contains their convex hull $C$. Conversely, $D$ is clearly included in $C$, and it is easy to check that $I C \subset C$ (because $I\left\{v_{i}\right\} \subset C$ for all $i$, see for example $\left.[\mathbf{A S}]\right)$. So $I D \subset C$ and, by closedness, $\overline{I D}=C$. Clearly, $D \subset \partial \overline{I D}$ because no two 
points of $D$ are collinear. So $D \subset \partial C$ as sets, and hence as polyhedral complexes because the bending angles of $D$ are nonzero. So we only need to prove

Lemma 9.2. The codimension-1 simplicial complex $D \subset \mathbb{R}^{4}$ is locally convex $(\vec{u}$ pointing inward $)$.

Proof. Consider adjacent ideal tetrahedra $\Delta, \Delta^{\prime}$ in $\mathbb{H}^{3}$, which are lifts from tetrahedra $\Delta_{i-1}, \Delta_{i}$ of the manifold. We must prove that the dihedral angle in $\mathbb{R}^{4}$ between $\tau_{\Delta}$ and $\tau_{\Delta^{\prime}}$ points "downward". We will assume that the letter between $i-1$ and $i$ is an $L$ belonging to a subword $R L^{n} R$ of $\Omega$. In the link of the cusp, the pleated surface $S_{i}$ between $\Delta_{i-1}$ and $\Delta_{i}$ contributes a broken line $\left(-1, \zeta, \zeta^{\prime}, 1\right)$ in $\mathbb{C}$ together with its iterated images under $u \mapsto u \pm 2$, as in Figure 10 (we assume that the vertices $-1,1$ both belong to the base segments of the Euclidean triangles just below and just above the broken line, in the sense of Figure 5). We use the notation

$$
\begin{aligned}
\zeta+1 & =\vec{a}=a e^{i A} \\
\zeta^{\prime}-\zeta & =\vec{b}=b e^{i B} \\
1-\zeta^{\prime} & =\vec{c}=c e^{i C}
\end{aligned}
$$

(so far $A, B, C$ are only defined modulo $2 \pi$ ). Above this broken line in the upper half-space model lives a lift of $S_{i}$, containing the ideal triangles $(-1, \zeta, \infty$,$) and \left(\zeta, \infty, \zeta^{\prime}\right)$ and $\left(\infty, \zeta^{\prime}, 1\right)$, which admits as a deck transformation

$$
f: u \mapsto 1+\frac{(\zeta+1)\left(\zeta^{\prime}-1\right)}{u+1}
$$

because $f$ maps the first of the above mentioned triangles to the last (respecting the order of the vertices). Therefore, $f\left(H_{1, \infty}\right)=H_{|\zeta+1|\left|\zeta^{\prime}-1\right|, 1}=$ $H_{a c, 1}$. Note that $a$ and $c$ are the lengths of the segments of the broken line adjacent to the vertex 1 . Similarly, the following horoballs are all images of one another by deck transformations:

$$
H_{1, \infty} ; H_{a c,-1} ; H_{a b, \zeta} ; H_{b c, \zeta^{\prime}} ; H_{a c, 1} \text {. }
$$

If $\zeta=\xi+\eta \sqrt{-1}$ and $\zeta^{\prime}=\xi^{\prime}+\eta^{\prime} \sqrt{-1}$, the corresponding isotropic vectors in Minkowski space are respectively

$$
\begin{aligned}
& v_{\infty}=\left(\begin{array}{cccc}
0, & 0, & -1, & 1
\end{array}\right) \\
& v_{-1}=\frac{1}{a c}\left(\begin{array}{cccc}
-2, & 0, & 0, & 2
\end{array}\right)
\end{aligned}
$$

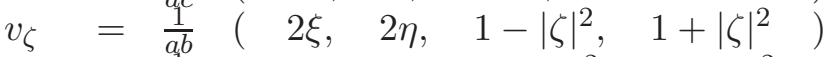

$$
\begin{aligned}
& v_{\zeta^{\prime}}=\frac{1}{b c}\left(2 \xi^{\prime}, 2 \eta^{\prime}, 1-\left|\zeta^{\prime}\right|^{2}, 1+\left|\zeta^{\prime}\right|^{2}\right) \\
& v_{1}=\frac{1}{a c}(2, \quad 0, \quad 0, \quad 2 \quad) \text {. }
\end{aligned}
$$

Observe that the hyperplane through $0, v_{\zeta}, v_{\zeta^{\prime}}, v_{\infty}$ separates $v_{1}$ from $v_{-1}$ because the hyperbolic plane through the ideal points $\zeta, \zeta^{\prime}, \infty \in \mathbb{P}^{1} \mathbb{C}$ separates the ideal points 1 and -1 . Also, observe that the imaginary 


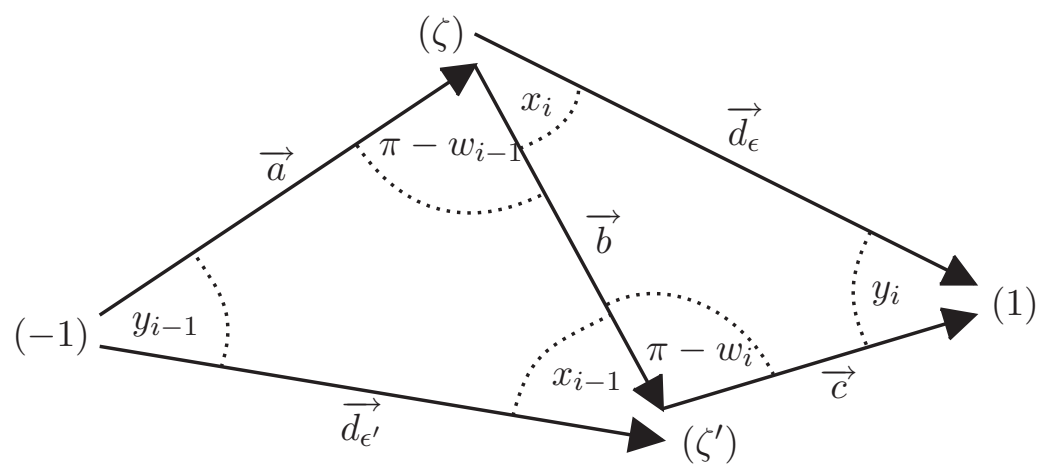

Figure 10. Adjacent tetrahedra $\Delta_{i-1}, \Delta_{i}$ (cusp view).

parts $\eta, \eta^{\prime}$ of $\zeta, \zeta^{\prime}$ satisfy $\eta>\eta^{\prime}$ because the Euclidean triangles $-1 \zeta^{\prime} \zeta$ and $1 \zeta \zeta^{\prime}$ are counterclockwise oriented.

To prove that the dihedral angle at the codimension-2 face projecting to $\left(\zeta \zeta^{\prime} \infty\right)$ is convex, it is enough to show that if $P v_{\zeta}+Q v_{\zeta^{\prime}}+R v_{\infty}=$ $\lambda v_{1}+(1-\lambda) v_{-1}$ (for some $0<\lambda<1$ ), then $P+Q+R>1$ (moreover, this will, in fact, take care of all codimension- 2 faces of the simplicial complex $D$ ). One easily finds the unique solution

$$
P=\frac{-b \eta^{\prime}}{c\left(\eta-\eta^{\prime}\right)} ; Q=\frac{b \eta}{a\left(\eta-\eta^{\prime}\right)} ; R=\frac{\eta\left(1-\left|\zeta^{\prime}\right|^{2}\right)-\eta^{\prime}\left(1-|\zeta|^{2}\right)}{a c\left(\eta-\eta^{\prime}\right)}
$$

(the value of $\lambda$ will not matter to us); hence

$$
\begin{aligned}
P+Q+R & =1+\frac{Z}{a c\left(\eta-\eta^{\prime}\right)} \\
& \text { where } Z=b c \eta-a b \eta^{\prime}+\eta\left(1-\left|\zeta^{\prime}\right|^{2}\right)-\eta^{\prime}\left(1-|\zeta|^{2}\right)+a c\left(\eta^{\prime}-\eta\right) .
\end{aligned}
$$

So it is enough to prove that $Z>0$. Endow $\mathbb{C} \simeq \mathbb{R}^{2}$ with the usual scalar product "•" and observe that $1-|\zeta|^{2}=\vec{a} \bullet(\vec{b}+\vec{c})$ and $1-\left|\zeta^{\prime}\right|^{2}=$ $(\vec{a}+\vec{b}) \bullet \vec{c}$. Hence

$$
\begin{aligned}
Z=\eta(b c+\vec{b} \bullet \vec{c})-\eta^{\prime}(a b+\vec{a} \bullet \vec{b})+\left(\eta^{\prime}-\eta\right)(a c-\vec{a} \bullet \vec{c}) \\
=a b c\left[\frac{\eta}{a}(1+\cos (B-C))-\frac{\eta^{\prime}}{c}(1+\cos (A-B))\right. \\
\left.\quad+\frac{\eta^{\prime}-\eta}{b}(1-\cos (A-C))\right] \\
=a b c[\sin A(1+\cos (B-C))+\sin C(1+\cos (A-B)) \\
\quad+\sin B(1-\cos (A-C))] \\
=4 a b c \sin \frac{A+C}{2} \cos \frac{A-B}{2} \cos \frac{B-C}{2}
\end{aligned}
$$


by standard trigonometric formulae. Observe that the last expression is a well-defined function of $A, B, C \in \mathbb{R} / 2 \pi \mathbb{Z}$ (although each factor is defined only up to sign). Next, however, we shall carefully pick representatives of $A, B, C$ in $\mathbb{R}$.

Observation 9.3. There exists a unique triple of representatives $(A, B, C) \in \mathbb{R}^{3}$ such that $\{A, B, C\} \subset J$ where $J$ is an open interval of length less than $\pi$, containing 0 . (Indeed, the broken line $\left(\ldots,-1, \zeta, \zeta^{\prime}\right.$, $1, \ldots)$ invariant under $u \mapsto u \pm 2$ has no self-intersection, and this fact is equivalent to the existence of an open half-plane $H$ containing the vectors $\vec{a}, \vec{b}, \vec{c}$ : the observation follows since $\vec{a}+\vec{b}+\vec{c}=2$ belongs to $H$.)

We pick the representatives $A, B, C \in \mathbb{R}$ given by Observation 9.3, and do the same for the broken line contributed by each surface $S_{i}$ for $i \in \mathbb{Z}$ (each broken line, being included in an infinite horizontal strip, can be oriented from $-\infty$ to $+\infty$, so each edge $\epsilon$ of the cusp link inherits an orientation, as in Figure 10: we write its complex coordinate $\left.\overrightarrow{d_{\epsilon}}=d_{\epsilon} e^{D_{\epsilon}}\right)$. By Observation 9.3, all the complex arguments $D_{\epsilon}$ are in $(-\pi, \pi)$. But since $0 \leq w \leq \pi$, the existence of the interval $J$ also implies (in the notation of Figure 10):

$$
A=B+w_{i-1} \text { and } C=B+w_{i} ;
$$

hence $B=\inf \{A, B, C\}$ and $B \in(-\pi, 0)$. It follows that $B+x_{i} \in$ $(-\pi, \pi)$, hence (writing $\epsilon=[\zeta, 1]$ and $\epsilon^{\prime}=\left[-1, \zeta^{\prime}\right]$ as in Figure 10):

$$
D_{\epsilon}=B+x_{i} \text { and similarly } D_{\epsilon^{\prime}}=B+x_{i-1} \text {. }
$$

In other words (by transitivity), for any two edges $\epsilon_{1}, \epsilon_{2}$ of the cusp link, the difference of arguments $D_{\epsilon_{1}}-D_{\epsilon_{2}} \in \mathbb{R}$ can be read off "naively" as a linear combination of the $\left\{w_{i}\right\}_{i \in \mathbb{Z}}$, with no multiple of $2 \pi$ added.

Now that all arguments are fixed in the real interval $(-\pi, \pi)$, we can see that $\cos \frac{A-B}{2}=\cos \left(w_{i-1} / 2\right)$ and $\cos \frac{B-C}{2}=\cos \left(w_{i} / 2\right)$ are positive. So to prove $Z>0$, it remains to see that $0<A+C<2 \pi$. This in turn follows from a small variant of Lemma 16 in $[\mathbf{G F}]$, which we prove now (it implies an empirical observation of Jørgensen which was Conjecture 8.6 in $[\mathbf{A S W Y 2}])$.

Proposition 9.4. With the above notation, one has $0<A+C<2 \pi$.

Consider the full subword $R L^{n} R$, with the broken line $\left(-1, \zeta, \zeta^{\prime}, 1\right)$ corresponding to some $L$. There is, in fact, a sequence of points $\left(\zeta_{0}, \zeta_{1}\right.$, $\left.\ldots, \zeta_{n}\right)$ in $\mathbb{C}$ such that the broken line corresponding to the $j$-th letter $L$ is $\left(-1, \zeta_{j}, \zeta_{j-1}, 1\right)$ for all $1 \leq j \leq n$. The broken line corresponding to the initial (resp. final) $R$ is $\left(-1, \zeta_{0}, \zeta_{-1}, 1\right)$ for some $\zeta_{-1}$ (resp. $\left(-1, \zeta_{n+1}, \zeta_{n}, 1\right)$ for some $\left.\zeta_{n+1}\right)$. There exists $1 \leq k \leq n$ such that $\left(\zeta, \zeta^{\prime}\right)=\left(\zeta_{k}, \zeta_{k-1}\right)$ 


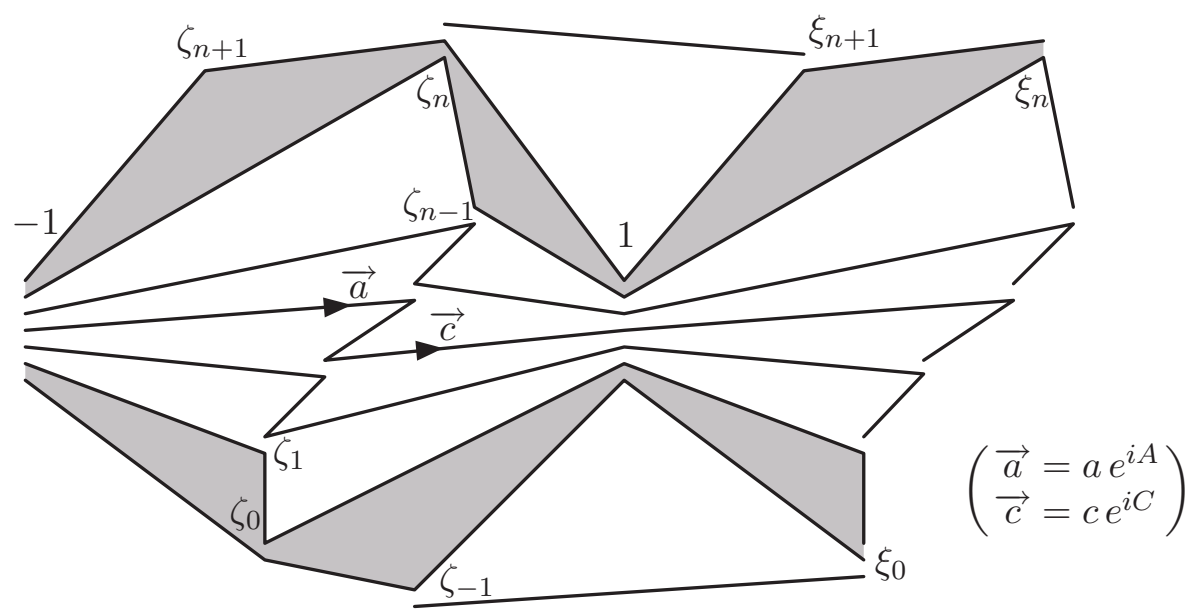

Figure 11. Cusp view: a subword $R L^{n} R$, bounded by hinge indices 0 and $n$ (grey).

Set $\xi_{j}:=\zeta_{j}+2$ for all $j$. By construction, the rays issued from 1 through $\zeta_{-1}, \zeta_{0}, \ldots, \zeta_{n-1}, \zeta_{n}, \xi_{n+1}, \xi_{n}, \ldots, \xi_{1}, \xi_{0}, \zeta_{-1}$ (in that cyclic order) divide $\mathbb{C}$ clockwise into angular sectors whose measures are less than $\pi$ and add up to $2 \pi$ (these $2 n+4$ rays realize the link of the vertex 1 in the cusp triangulation, see Figure 11). Comparing the angles at 1 and at -1 , and denoting segments $\epsilon$ of the cusp link by their endpoints (and their complex arguments by $D_{\epsilon}$ ), we see immediately that $D_{-1 \zeta_{k+1}}-D_{-1 \zeta_{k}}=D_{\zeta_{k-1} 1}-D_{\zeta_{k} 1}$. Therefore,

(21) $A+C=D_{-1 \zeta_{k}}+D_{\zeta_{k-1} 1}=D_{-1 \zeta_{k+1}}+D_{\zeta_{k} 1}=\cdots=D_{-1 \zeta_{j}}+D_{\zeta_{j-1} 1}$

(for all $0 \leq j \leq n+1$, by induction). Since the triangles $\zeta_{0} \zeta_{-1} 1$ and $\xi_{0} 1 \zeta_{-1}$ are counterclockwise oriented (and $\xi_{0}=\zeta_{0}+2$ ), we have $\operatorname{Im}\left(\zeta_{-1}\right)<\operatorname{Im}(1)$, i.e., $\operatorname{Im}\left(\zeta_{-1}\right)<0$. Similarly, $\zeta_{n} 1 \xi_{n+1}$ and $\xi_{n} \xi_{n+1} 1$ are counterclockwise oriented, $\operatorname{so} \operatorname{Im}\left(\xi_{n+1}\right)>0$. So there exists $0 \leq j \leq n+1$ such that $\operatorname{Im}\left(\zeta_{j-1}\right)<0$ and $\operatorname{Im}\left(\zeta_{j}\right) \geq 0$. This implies $D_{-1 \zeta_{j}} \in(0, \pi)$ and $D_{\zeta_{j-1} 1} \in[0, \pi)$. By $(21)$, this implies $A+C \in(0,2 \pi)$. Theorem 9.1 is proved.

q.e.d.

In $[\mathbf{G F}]$, we studied punctured-torus bundles over the circle by indexing the tetrahedra $\Delta_{i}$ in $\mathbb{Z} / m \mathbb{Z}$ (instead of $\left.\mathbb{Z}\right)$ : note that the proof of Theorem 9.1 applies without alteration to that context.

\section{Generalizations}

In this section, we extend all the previous results to punctured-torus groups with rational pleatings and/or infinite ends. By an admissible lamination $\lambda$, we mean any of the following objects: 
- An ordered pair (up to sign) $\lambda= \pm(\alpha, \beta) \in \mathbb{R}^{* 2} / \pm$ such that $\beta / \alpha$ is irrational.

- An ordered pair $\lambda= \pm(\theta \alpha, \theta \beta)$ where $\alpha, \beta \in \mathbb{Z}$ are coprime and $0<\theta<\pi$ (we call $\theta$ the weight of $\lambda$ and write $|\lambda|=\theta$ ).

- An ordered pair $\lambda= \pm(\pi \alpha, \pi \beta)$ where $\alpha, \beta \in \mathbb{Z}$ are coprime (we call $\pi$ the weight of $\lambda$ and write $|\lambda|=\pi)$.

- A real projective class $\lambda=\{(\mu \alpha, \mu \beta) \mid \mu \neq 0\}$ where $\beta / \alpha$ is irrational.

The slope of the admissible lamination is the number $s(\lambda)=\beta / \alpha \in \mathbb{P}^{1} \mathbb{R}$. Each end $\omega$ of a punctured-torus group can be associated a unique (ending or pleating) admissible lamination: the four cases above correspond respectively to $\omega$ being

- finite, with irrational pleating lamination;

- finite, with rational pleating lamination and pleating angle $\theta$;

- finite, with a rational ending lamination (an accidental cusp);

- infinite, with an irrational ending lamination (see [B1] and Section 3 of [Min] for a definition).

Theorem 10.1. Let $\lambda^{+}, \lambda^{-}$be admissible laminations of distinct slopes. There exists a punctured-torus group $\Gamma$ with ending and/or pleating laminations $\lambda^{ \pm}$, and the interior $V$ of the convex core of $\mathbb{H}^{3} / \Gamma$ has an ideal decomposition $\mathcal{D}=\mathcal{D}^{\text {Comb }}$ into polyhedral cells (of positive volume) whose combinatorics are given by $\lambda^{ \pm}$in the following sense: if $\Lambda$ is the line from $s\left(\lambda^{-}\right)$to $s\left(\lambda^{+}\right)$across the Farey diagram in $\mathbb{H}^{2}$, then

(i) If $s\left(\lambda^{+}\right)$and $s\left(\lambda^{-}\right)$are irrational, $\mathcal{D}$ consists of ideal tetrahedra $\left(\Delta_{i}\right)_{i \in \mathbb{Z}}$ in natural bijection with the Farey edges crossed by $\Lambda$, as in Section 1.

(ii) If only $s\left(\lambda^{+}\right)$is rational and $\left|\lambda^{+}\right|<\pi$, then $\mathcal{D}$ has one ideal tetrahedron per Farey edge crossed by $D$, and one cell $T$ whose interior is non-contractible, homeomorphic to a solid torus: $\partial_{1}:=$ $\overline{\partial T \cap \partial V}$ is a punctured torus pleated along a simple closed curve of slope $s\left(\lambda^{+}\right)$, and $\partial_{2}:=\overline{\partial T \backslash \partial V}$ is a punctured torus pleated along the ideal triangulation associated to the Farey triangle with vertex $s\left(\lambda^{+}\right)$crossed by $\Lambda$. Finally, $\partial_{1} \cap \partial_{2}$ is a line from the puncture to itself of slope $s\left(\lambda^{+}\right)$. See the left panel of Figure 12.

(iii) If only $s\left(\lambda^{+}\right)$is rational and $\left|\lambda^{+}\right|=\pi$, all the statements of the previous case apply, except that $\partial_{1}=\overline{\partial T \cap \partial V}$ becomes a thricepunctured sphere (the simple closed curve of slope $s\left(\lambda^{+}\right)$has been "pinched" to become a cusp): see the right panel of Figure 12.

(iv) If only $s\left(\lambda^{-}\right)$is rational, the situation is similar to the previous two cases, exchanging $\lambda^{-}$and $\lambda^{+}$.

(v) If $s\left(\lambda^{+}\right), s\left(\lambda^{-}\right)$are rationals but not Farey neighbors, the situation is again similar, with two solid torus cells $T^{+}$and $T^{-}$. 
(vi) If $s\left(\lambda^{+}\right), s\left(\lambda^{-}\right)$are Farey neighbors, $\mathcal{D}$ only consists of two solid tori $T^{+}$and $T^{-}$as above, glued along a punctured torus $S$ pleated along only two (not three) lines from the cusp to itself, of slopes $s\left(\lambda^{+}\right)$and $s\left(\lambda^{-}\right)$.

Moreover, $\mathcal{D}$ agrees with the geometrically canonical decomposition $\mathcal{D}^{\text {Geom }}$ of $V$ given by the Epstein-Penner convex hull construction.

Note that the combinatorics of $\mathcal{D}^{\text {Geom }}$ does not depend on the nature (finite or infinite) of the ends of $\mathbb{H}^{3} / \Gamma$. At this point, we have treated the case of two irrational pleatings (finite ends). We proceed to prove the remaining cases of Theorem 10.1.
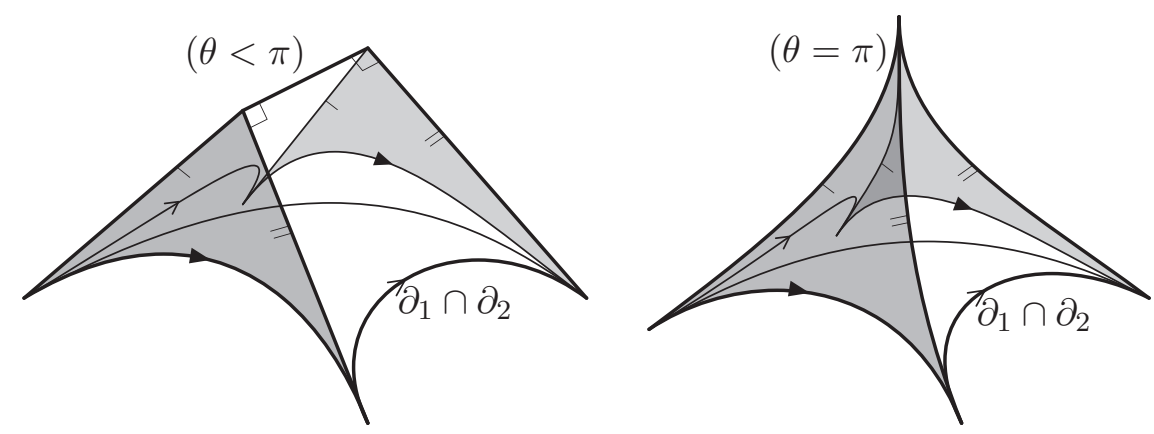

Figure 12. Toric cells: the exterior dihedral angle $\theta$ is the weight $\left|\lambda^{ \pm}\right|$. Shaded faces are identified, as well as edges carrying identical arrows.

10-A. One rational pleating. We focus on the case of two finite ends with only $\beta^{+} / \alpha^{+}$rational. We can choose to end the word $\Omega \in\{R, L\}^{\mathbb{Z}}$ with an infinite suffix $L R R \ldots R \ldots$ (or of $R L L \ldots L \ldots$... that is an arbitrary choice) and proceed from Section 1 onward. We shall assume that $i=0$ is the greatest hinge index. Sections 2 through 3-A are unchanged: the sequence $\left(w_{i}\right)$ is just concave (thus convergent and non-decreasing) on $\mathbb{N}$. In Subsection $3-\mathrm{B}$, we find that the sequence $\left(\phi_{i}^{+}\right)$is constant on $\mathbb{N}$, equal to some positive real. By (9), this real is the weight $\theta$ of the rational lamination $\lambda^{+}$, so we assume $\theta<\pi$. Section 4 goes through essentially unchanged: by the computation of Sublemma 4.2 (and with the same notation), the sum of the volumes of all tetrahedra $\Delta_{i}$ for $i \geq 2^{n}$ is at most

$$
\sum_{k>n} \Sigma_{2^{k-1}}^{2^{k}} \leq \sum_{k>n} 2^{-k}[1+(2 k-1) \log 2]=O\left(2^{-n / 2}\right) .
$$

Therefore, the volume functional $\mathcal{V}$ is bounded, continuous for the product topology, and concave. We can find a maximizer $w$ of $\mathcal{V}$, and it still satisfies Propositions 4.4 and 4.5: in particular, all tetrahedra $\Delta_{i}$ for $i>0$ have positive angles. 
Something new is required from Section 5 on: we must prove the identity $\lim _{i \rightarrow+\infty} w_{i}=\theta$. The $\left\{w_{i}\right\}_{i \geq 2}$ contribute only to the angles of the $\left\{\Delta_{i}\right\}_{i \geq 1}$, which are positive: so the volume $\mathcal{V}$ is critical with respect to each $w_{i}$ for $i \geq 2$. By Sublemma 6 of $[\mathbf{G F}]$, it follows that the cusp triangles of the $\left\{\Delta_{i}\right\}_{i \geq 1}$ fit together correctly and can be drawn in the Euclidean plane $\mathbb{C}$. More precisely, there exists a sequence of complex numbers $\left(\zeta_{i}\right)_{i \geq 0}$ such that for all $i \geq 1$, the triangles contributed by $\Delta_{i}$ have vertices at $\left(-1, \zeta_{i}, \zeta_{i-1}\right)$ and $\left(1, \zeta_{i}, \zeta_{i+1}\right)$ (Figure 13, left). These triangles being similar, we have $\left(\zeta_{i}+1\right)\left(\zeta_{i+1}-1\right)=\left(\zeta_{i-1}+1\right)\left(\zeta_{i}-1\right)=$ $\cdots=\left(\zeta_{0}+1\right)\left(\zeta_{1}-1\right)$; hence

$$
\zeta_{i+1}=\frac{\zeta_{i}+\kappa}{\zeta_{i}+1}=: \varphi\left(\zeta_{i}\right)
$$

for some complex number $\kappa \neq 1$ independent of $i$. Observe that the complex length $\ell$ of the hyperbolic isometry (extending) $\varphi$ satisfies $\cosh \ell=\frac{1+\kappa}{1-\kappa}$. In the end, $\varphi$ will be a lift of the loop along the rational pleating line of the convex core.

Proposition 10.2. The number $\kappa$ lies in the real interval $(0,1)$.

Proof. Let $Z_{i}$ be the $(u \mapsto u \pm 2)$-invariant broken line $\left(\ldots,-1, \zeta_{i-1}, \zeta_{i}\right.$, $1, \ldots$ ) contributed by the pleated surface $S_{i}$. Following $(2)$, recall that: (22)

$$
\left\{\begin{array}{l}
\text { The angle of } Z_{i} \text { above } \zeta_{i-1} \text { is } \pi-w_{i} ; \\
\text { The angle of } Z_{i} \text { above } \zeta_{i} \text { is } \pi+w_{i-1} \\
\text { The angle of } Z_{i} \text { above } 1 \text { (or }-1 \text { ) is } \alpha_{i}:=\pi+w_{i}-w_{i-1} \underset{i \rightarrow+\infty}{\longrightarrow} \pi .
\end{array}\right.
$$

First, $\kappa$ is real: if not, the $\zeta_{i}$ have a limit in $\mathbb{C} \backslash \mathbb{R}$ (a square root of $\kappa)$, so $\alpha_{i}$ cannot go to $\pi$. If $\kappa<0$, then $\varphi$ is a pure rotation: the $\zeta_{i}$ all belong to a circle of the open lower half-plane, which also contradicts $\alpha_{i} \rightarrow \pi$. If $\kappa>1$, the $\zeta_{i}$ go to $\pm \sqrt{\kappa}$ (and $\varphi$ is a gliding axial symmetry of $\mathbb{H}^{3}$ ) so that $\alpha_{i} \rightarrow 0$, likewise a contradiction. If $\kappa=0$, then $\varphi$ is a parabolic transformation fixing 0 : the $\zeta_{i}$ go to 0 along a circle tangent to $\mathbb{R}$, and one can see that $w_{i}=\widehat{\zeta_{i} \zeta_{i+1} 1}$ goes to $\pi>\lim _{+\infty} \phi^{+}=\theta$. The only remaining possibility is $\kappa \in(0,1)$, where $\varphi$ is a pure translation.

q.e.d.

Thus, the $\zeta_{i}$ lie on a circle arc $C$ of the lower half-plane which meets the real line at $\pm \sqrt{\kappa}$, and $\lim _{+\infty} \zeta_{i}=\sqrt{\kappa}$. We denote by $\pi-\theta^{*}$ the angle between $C$ and the ray $[\sqrt{\kappa},+\infty$ ) (more precisely, the angle between their half-tangents at $\sqrt{\kappa}$ ). It is easy to see that

$$
\theta^{*}=\lim _{i \rightarrow+\infty} w_{i}
$$

(see Figure 13). Hence, $\theta^{*} \leq \theta$.

Proposition 10.3. One has $\theta^{*}=\theta$. 

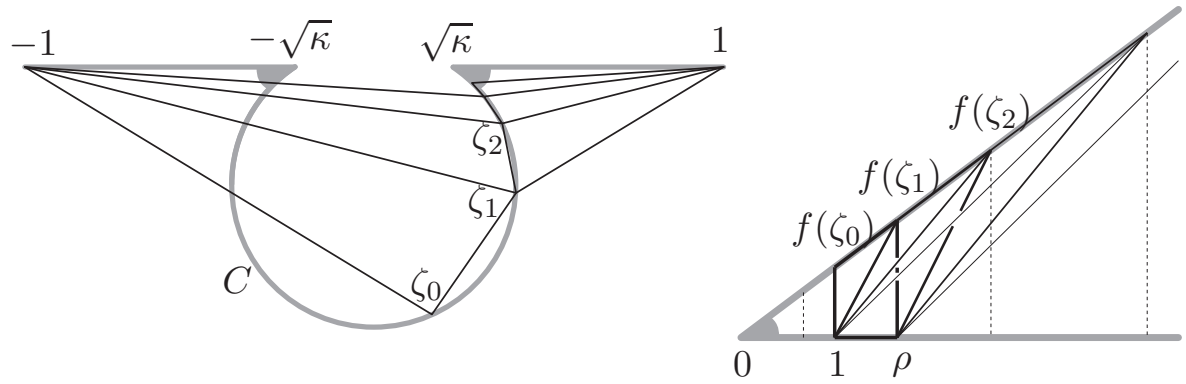

Figure 13. All marked angles (grey) are $\pi-\theta^{*}$, and $\zeta_{j+1}=\varphi\left(\zeta_{j}\right)=\frac{\zeta_{j}+\kappa}{\zeta_{j}+1}$.

Proof. The idea of the proof is to synthetically understand the deformation space of the toric cell (Figure 12, left), seen as a union of tetrahedra, whose volume will be a monotonous function of $\theta^{*}$. (In particular, this deformation space is independent of whether the triangulation of the toric cell is induced by a suffix $R L L \ldots L \ldots$ or $L R R \ldots R \ldots)$.

First, let us check that any data $0 \leq w_{0}<w_{1}<\theta^{*}<\pi$ smoothly determines a unique pair of complex numbers $\zeta_{0}, \zeta_{1}$ such that:

(i) the $(u \mapsto u \pm 2)$-invariant broken line $Z_{1}=\left(\ldots,-1, \zeta_{0}, \zeta_{1}, 1, \ldots\right)$ has angles $\left(w_{0},-w_{1}, w_{1}-w_{0}\right)$, as in (2) and (22) above;

(ii) the number $\kappa$ such that $\zeta_{1}=\frac{\zeta_{0}+\kappa}{\zeta_{0}+1}$ lies in $(0,1)$;

(iii) the circle $C$ through $\zeta_{0}$ and $\zeta_{1}$ centered on the imaginary axis intersects the real axis at an angle $\theta^{*}$.

Indeed, (ii) means that $\zeta_{0}+1$ and $1-\zeta_{1}$ have opposite arguments, so one must look for $\zeta_{0}$ on the ray $-1+e^{\frac{w_{0}-w_{1}}{2} i} \mathbb{R}_{+}^{*}$, and $\zeta_{1}$ on $1-$ $e^{\frac{w_{1}-w_{0}}{2} i} i \mathbb{R}_{+}^{*}$. These rays intersect at a point $p$ of the negative imaginary axis, and the line $\zeta_{0} \zeta_{1}$ is parallel to $e^{\frac{w_{0}+w_{1}}{2} i} \mathbb{R}$. In the limit case as $\zeta_{1}$ goes to 1 , it is easy to see that the angle between the circle $C$ and the real axis goes to $w_{1}$ : thus, as $\zeta_{1}$ goes away from 1 , this angle ranges smoothly monotonically over $\left(w_{1}, \pi\right)$ (and the triple $\left(\zeta_{0}, \zeta_{1}, C\right)$ undergoes a homothetic contraction of center $p)$.

These $\zeta_{0}, \zeta_{1}$ in turn define all $\left\{\zeta_{j}\right\}_{j \geq 2}$ via $\zeta_{j+1}=\varphi\left(\zeta_{j}\right)=\frac{\zeta_{j}+\kappa}{\zeta_{j}+1}$, and we can read off the angle $w_{j}={\widehat{\zeta_{j+1} \zeta_{j}}} \leq \theta^{*}$ and construct the associated ideal tetrahedron $\Delta_{j}$. In what follows, we investigate the shape of the space $U:=\bigcup_{j \geq 1} \Delta_{j}$, whose metric boundary (the punctured torus $S_{1}$ ) has pleating angles $\left(w_{0},-w_{1}, w_{1}-w_{0}\right)$ (from (2) above).

Define $f(\zeta):=\frac{\zeta+\sqrt{\kappa}}{\zeta-\sqrt{\kappa}}$, so that $f(\varphi(\zeta))=\rho f(\zeta)$ where $\rho:=\frac{1+\sqrt{\kappa}}{1-\sqrt{\kappa}}$. The hyperbolic convex hull of $\left(\infty, 1, \zeta_{j}, \zeta_{j+1}\right)$ is isometric to the tetrahedron $\Delta_{j}$ : pushing forward by $f$, we obtain a tetrahedron $\Delta_{j}^{\prime}$, isometric to $\Delta_{j}$, with vertices $\left(1, \rho, \rho^{j} f\left(\zeta_{0}\right), \rho^{j+1} f\left(\zeta_{0}\right)\right)$ (Figure 13, right). Moreover, all 
the $f\left(\zeta_{j}\right)=\rho^{j} f\left(\zeta_{0}\right)$ lie on the half-line $e^{i\left(\pi-\theta^{*}\right)} \mathbb{R}^{+}$. By reasoning in a fundamental domain of the loxodromy $\Phi: u \mapsto \rho u$, it is then easy to see that $U$ has the same volume as $D:=D_{1} \cup D_{2}$, where $D_{1}, D_{2}$ are ideal tetrahedra of vertices $\left(\infty, f\left(\zeta_{1}\right), 1, \rho\right)$ and $\left(\infty, f\left(\zeta_{1}\right), 1, f\left(\zeta_{0}\right)\right)$ respectively. Moreover, $\Phi$ identifies the faces $\left(\infty, 1, f\left(\zeta_{0}\right)\right)$ and $\left(\infty, \rho, f\left(\zeta_{1}\right)\right)$ of $D$, so that $D / \Phi$ is a manifold with polyhedral boundary, homeomorphic to a solid torus, with interior dihedral angles

$$
\left(\pi-w_{1}, \pi+w_{0}, \frac{w_{1}-w_{0}}{2}, \frac{w_{1}-w_{0}}{2}, \pi-\theta^{*}\right) .
$$

The edge of $D / \Phi$ with dihedral angle $\pi-\theta^{*}$ is a simple closed curve of length $\log \rho$, toward which $D_{1}, D_{2}$ spiral. A picture of $D / \Phi$ is obtained by replacing $\theta$ with $\theta^{*}$ in the left panel of Figure 12 .

Using the smooth dependence on $\theta^{*}$ (and fixing $w_{0}, w_{1}$ ), the Schläfli volume formula then gives $\partial \mathcal{V}(D / \Phi) / \partial \theta^{*}=\frac{1}{2} \log \rho>0$ : so $D / \Phi$ (and therefore $U)$ has largest volume when $\theta^{*}$ is largest. Regard the $\left\{w_{j}\right\}_{j \leq 1}$ as fixed, and the $\left\{w_{j}\right\}_{j \geq 2}$ as unknowns: then $\left(w_{2}, w_{3}, \ldots\right)$ is clearly the solution to the maximization problem for the volume of $U$ (with fixed pleating angles on $S_{1}$ ). Therefore, the $w_{i}$ will choose the largest possible limit $\theta^{*}$ at $+\infty$, namely $\theta^{*}=\theta$.

q.e.d.

The above proof does more than determining $\lim _{+\infty} w_{j}$ : as in Sections 5 through 8, it gives a full description of $U=\bigcup_{j \geq 1} \Delta_{j}$ and of its boundary (whose pleating on the outer side turns out to be $\lambda^{+}$). Here is, however, an important observation:

Observation 10.4. Proposition 9.4 above, " $A+C>0$ ", does not hold for the family of pleated surfaces $\left(S_{i}\right)_{i \geq 0}$. Instead, we have $A+C=$ 0 . This simply means that $\overrightarrow{-1, \zeta_{i}}$ and $\overrightarrow{\zeta_{i+1}, 1}$ make opposite angles with the real line. Indeed, $\left(\zeta_{i}+1\right)\left(1-\zeta_{i+1}\right)=1-\kappa$ is a positive real.

We can now establish

Proposition 10.5. All the (strict) inequalities of (11) are true.

Proof. This is Proposition 4.6 (in the new context where $\beta^{+} / \alpha^{+}$is rational). The proof is the same, with the following caveat: in ruling out $w_{j}=0$ for $j$ hinge, we call upon $[\mathbf{G F}]$ (especially Lemma 16 and the argument of Section 9 there). The strategy is to assume $w_{j}=$ 0 , and then perturb $w$ to a well-chosen $w^{\varepsilon}$ so as to make the volume increase: $\partial \mathcal{V} / \partial \varepsilon>0$. The latter inequality holds essentially because the inequality of Proposition $9.4(" A+C>0$ ") is true, both in the $R^{n}$-word preceding $j$ and in the $L^{m}$-word following $j$. (Note: in Section 9 of $[\mathbf{G F}]$, the inequality " $A+C>0$ " is formulated in terms of lengths and takes the guise " $Q<P+T$ ".) More exactly, $\partial \mathcal{V} / \partial \varepsilon$ will be positive when at least one of the two instances of " $A+C \geq 0$ " is strict. But it is always strict, except in a single case (the infinite suffix $L R R \ldots R \ldots$ ): so we can conclude.

q.e.d. 
As a result, all the tetrahedra $\Delta_{i}$ have positive angles and fit together correctly: Sections 5-8 carry through for the $\lambda^{-}$-end, and $\bigcup_{i \in \mathbb{Z}} \Delta_{i}$ is the interior of the convex core of a quasifuchsian punctured-torus group, with the prescribed pleatings $\lambda^{ \pm}$.

The results of Section 9 extend readily: the only modification is that tetrahedra $\left(\Delta_{i}\right)_{i \geq 0}$ lift to a family of coplanar cells in Minkowski space, because the key inequality of Proposition 9.4 has become an equality. Therefore, the geometrically canonical decomposition of the interior of the convex core contains the non-contractible cell $D / \Phi$ (Figure 12, left).

10-B. Two rational pleatings. When both $\beta^{+} / \alpha^{+}$and $\beta^{-} / \alpha^{-}$are rational, the encoding word $\Omega \in\{R, L\}^{\mathbb{Z}}$ can be chosen with an infinite prefix $\ldots R \ldots R R L$ and an infinite suffix $L R R \ldots R \ldots$ We apply the same argument as above to both ends simultaneously. Again, Proposition 10.5 holds because no hinge index $j$ belongs both to the prefix and to the suffix.

If the rational pleating slopes $s\left(\lambda^{ \pm}\right)$are not Farey neighbors, we can convert the prefix to ...LLR and/or the suffix to $R L L \ldots$, obtaining different triangulations of (the interior of) the convex core of the same quasifuchsian group.

If $s\left(\lambda^{+}\right), s\left(\lambda^{-}\right)$are Farey neighbors, then $\Omega=\ldots R R L R R \ldots$ (observe that prefix and suffix overlap, so the two word conversions do not commute). If the indices $i$ before and after the central $L$ are 0 and 1 , we obtain $w_{0}=w_{1}$ by applying Observation 10.4 to prefix and suffix. In other words, the points $a, b, c$ in Figure 14 (left) are collinear. It is therefore possible to triangulate the same convex core according to a word $\Omega=\ldots L L L R R R \ldots$, provided that we allow the hinge tetrahedron $\Delta_{0}$ to become flat (black in Figure 14, right). In any case, the sequence $\left(w_{i}\right)_{i \in \mathbb{Z}}$ maximizes the total volume (this is the only case where any inequality of (11) becomes an equality for the volume maximizer $w$ ).

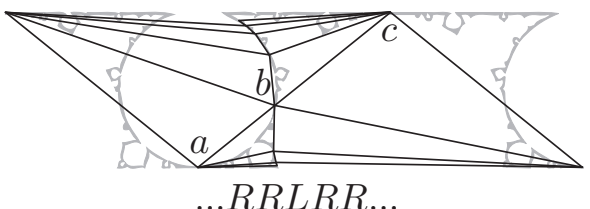

...RRLRR...

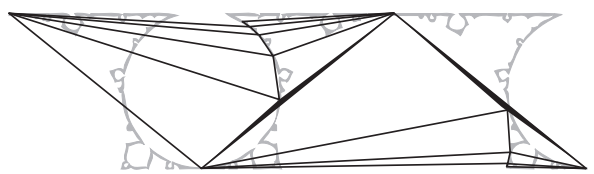

...LLLRRR ..

Figure 14. Two triangulations seen against the same limit set. In the right panel, a triangle flattens.

10-C. Pinching. The case where one (or both) of the pleatings $\lambda^{ \pm}$has weight $\pi$ is a straightforward limit case of Subsections 10-A and 10-B (the term "pinching" refers to the fact that the pleating curve becomes shorter and shorter, and eventually turns into a cusp as the pleating angle reaches $\pi$ ). 
Suppose $\left|\lambda^{+}\right|=\pi$ (note that the conditions of (11) involving $\phi^{+}$ become vacuous, because $\phi^{+} \geq \pi$ ). Subsection 10-A carries through with $\theta=\pi$ and $\kappa=0$. The circle arc $C$ of Figure 13 becomes a full circle, tangent to $\mathbb{R}$ at 0 . The analysis of Proposition 10.3 (existence and uniqueness of $\zeta_{0}, \zeta_{1} \in \mathbb{C}$ ) extends smoothly to $\theta^{*}=\pi$. One then finds that the tetrahedron with vertices $\left(\infty, 1, \zeta_{j}, \zeta_{j+1}\right)$ is sent by $f$ : $u \mapsto 1 / u$ to a tetrahedron $\Delta_{j}^{\prime}$ of $\mathbb{H}^{3}$, isometric to $\Delta_{j}$, with vertices $(0,1, \tau+j, \tau+j+1)$ for a certain $\tau \in \mathbb{C}$ independent of $j$ (in the right panel of Figure 13, the grey bounding rays are replaced by parallel lines). It is straightforward to check that $\bigcup_{j \geq 1} \Delta_{j}$ is the solid torus pictured in Figure 12 (right), and the Schläfli formula again implies that $\theta^{*}=\pi$ realizes the maximum volume.

After Proposition 10.3, the argument is unchanged.

10-D. Infinite ends. By the results of [Min], quasifuchsian groups are dense in the space of all discrete, faithful, type-preserving representations $\pi_{1}(S) \rightarrow$ Isom $^{+}\left(\mathbb{H}^{3}\right)$. In fact, a geometrically infinite end of such a representation comes with an ending lamination, namely an irrational projective measured lamination which should be thought of as an "infinitely strong pleating". In this section, we will assume $\beta^{+} / \alpha^{+} \notin \mathbb{P}^{1} \mathbb{Q}$ and consider $w^{T}$, the solution of the volume maximization problem for $\left(\phi^{-}, T \phi^{+}\right)$, where $T>0$ (namely, $w$ is subject to conditions (11), where $\phi^{+}$is replaced by $T \phi^{+}$; and Table (3) still expresses $\left.x_{i}, y_{i}, z_{i}\right)$.

In a discrete, type-preserving representation of a surface group, each compact measured lamination $\lambda$ on the surface receives a length, which can be computed by measuring the (weighted) lengths of weighted curves converging to $\lambda$. It is known $[\mathbf{B r}]$ that the lengths of the pleating laminations of a quasifuchsian group are bounded by a constant depending only on the underlying surface (here the punctured torus). By Thurston's double limit theorem (Theorems 4.1 and 6.3 of [ $\mathbf{T h}]$; see also $[\mathbf{O t}])$, the space of discrete, type-preserving representations in which two fixed measured laminations have length bounded by a given constant is compact. Therefore, up to taking a subsequence, the groups $\Gamma^{T}$ corresponding to $w^{T}$ converge algebraically to a certain $\Gamma$. By Theorem A of $[\mathrm{Min}], \mathbb{H}^{3} / \Gamma$ is homeomorphic to $S \times \mathbb{R}$ and must have an infinite end (otherwise, $\Gamma$ would be quasifuchsian and the volumes would stay bounded).

Proposition 10.6. The $\lambda^{-}$-end of $\mathbb{H}^{3} / \Gamma$ is finite, with pleating lamination $\lambda^{-}$. The $\lambda^{+}$-end is infinite, and its ending lamination is the projective class of $\lambda^{+}$.

Proof. By [B2] (Theorem D), the space of type-preserving representations of the abstract group $\Gamma$ is smoothly (in fact, holomorphically) parametrized by the data $(\tau, \omega)$ of a point $\tau$ of Teichmüller space $\mathcal{T}$, and a transverse $\mathbb{R} / 2 \pi \mathbb{Z}$-valued cocycle relative to a fixed topological lamination $\mu$ (such cocycles include pleating measures as special cases). Taking 
for $\mu$ the support of $\lambda^{-}$, we see that the moduli of the $\lambda^{-}$-boundaries of the convex cores of the $\Gamma^{T}$ must converge in $\mathcal{T}$. Therefore, $\mathbb{H}^{3} / \Gamma$ contains a locally convex pleated surface $H$ with pleating $\lambda^{-}$, which must be a boundary component of the convex core (the boundary at infinity of the universal cover of $H$ contains all parabolic fixed points).

The $\lambda^{+}$-end of $\mathbb{H}^{3} / \Gamma$ must therefore be infinite. The parabolic fixed points of the limit group $\Gamma$ determine a version of Figure 4 (a Euclidean cusp link), and therefore, a family of non-negative angle assignments $\left(w_{i}\right)_{i \in \mathbb{Z}}$ for the tetrahedra $\Delta_{i}$. By algebraic convergence, the $w^{T}$ converge to $w$ in the product topology. For any $i \leq j$, the total volume of $\Delta_{i-1}, \Delta_{i}, \ldots, \Delta_{j}, \Delta_{j+1}$ is maximal with respect to $w_{i}, \ldots, w_{j}$ : in particular, Propositions 4.4 and 4.5 are still true. The techniques of [GF] (Lemma 16 and Section 9 there) show that all $\Delta_{i}$ have positive angles, and Proposition 9.4, hence also Lemma 9.2, still hold: $\left\{\Delta_{i}\right\}_{i \in \mathbb{Z}}$ is the geometrically canonical decomposition of $\mathbb{H}^{3} / \Gamma$. In particular, the family of all edges of all tetrahedra $\left\{\Delta_{i}\right\}_{i \geq 0}$ forms a sequence of laminations which exits $\mathbb{H}^{3} / \Gamma$ : therefore, the projective class of $\lambda^{+}$is the end invariant.

q.e.d.

The case of two infinite ends is already treated in $[\mathbf{A k}]$. Theorem 10.1 is proved.

10-E. The Pleating Lamination Theorem for punctured-torus groups. Define the open set

$$
\mathcal{U}:=\left(\mathbb{R}^{2} \backslash \bigcup_{m, n \in \mathbb{Z}}\{(t m, t n) \mid t \geq \pi\}\right) / \pm
$$

(note that $0 \notin \mathcal{U}$ ). An admissible pleating lamination $\lambda$ can be identified with an element $\pm(\alpha, \beta)$ of $\mathcal{U}$. Also define

$$
\mathcal{L}:=\mathcal{U}^{2} \backslash\left\{\left( \pm\left(\alpha^{+}, \beta^{+}\right), \pm\left(\alpha^{-}, \beta^{-}\right)\right) \mid \frac{\beta^{+}}{\alpha^{+}}=\frac{\beta^{-}}{\alpha^{-}}\right\}
$$

the space of admissible pairs of pleating laminations.

It is well-known that the space $\mathcal{Q F}$ of quasifuchsian, non-fuchsian (punctured-torus) groups is a connected real manifold of dimension 4 , and that the "pleatings" define a continuous function from $\mathcal{Q F}$ to $\mathcal{L}$ (this last fact is very general, see $[\mathbf{K S}]$ ). Theorem 10.1 defines (in particular) a map in the other direction, $\Psi: \mathcal{L} \rightarrow \mathcal{Q F}$.

Proposition 10.7. The map $\Psi$ is continuous.

Proof. In other words, the representation $\rho: \Gamma \rightarrow \operatorname{Isom}^{+}\left(\mathbb{H}^{3}\right)$ constructed at the end of Section 4 (and extended to rational pleatings) is continuously parametrized by $\left(\lambda^{+}, \lambda^{-}\right)$. Our first observation is that if $\beta^{+} / \alpha^{+}$is rational, the initial choice of infinite prefix/suffix in Subsection 10-A does not change the resulting representation $\rho$ of $\Gamma$ up to 
conjugacy: it just induces different triangulations of the toric piece of Figure 12, whose deformation space is still the same. As a result, $\Psi$ is actually well-defined.

Suppose $\left(\alpha_{n}^{+}, \beta_{n}^{+}\right) \rightarrow\left(\alpha^{+}, \beta^{+}\right)$and $\left(\alpha_{n}^{-}, \beta_{n}^{-}\right) \rightarrow\left(\alpha^{-}, \beta^{-}\right)$in $\mathcal{U}$ (for convenience we will no longer write "up to the \pm -involution"), and define the oriented line $\Lambda_{n}$ from $\beta_{n}^{-} / \alpha_{n}^{-}$to $\beta_{n}^{+} / \alpha_{n}^{+}$across the Farey diagram. Also define the associated functions $\phi^{ \pm, n}$ as in (9), domains $W^{n}$ as in Definition 3.4, and solutions $\left(w_{i}^{n}\right)_{i \in \mathbb{Z}}$ to the volume maximization problem over $W^{n}$. If $\beta^{+} / \alpha^{+}$is rational, we may assume (up to restricting to two subsequences) that the $\left(\alpha_{n}^{+}, \beta_{n}^{+}\right)$converge to $\left(\alpha^{+}, \beta^{+}\right)$in the clockwise direction for the natural orientation of $\mathbb{P}^{1} \mathbb{R}$. We also make a similar assumption for $\beta^{-} / \alpha^{-}$.

A priori, the sequences $\left(w_{i}^{n}\right)_{i \in \mathbb{Z}}$ are defined only up to a shift of the index $i$. However, we can choose these shifts in a consistent way: there exists a Farey edge $e$ which is crossed by all the lines $\Lambda_{n}$ for $n$ large enough, so we decide that $w_{0}^{n}$ always lives on $e$ (namely, $e_{0}^{n}=e$ ). By compactness of $[0, \pi]^{\mathbb{Z}}$, some subsequence of $\left(w^{n}\right)_{n \in \mathbb{N}}$ converges to some $w^{*}$ in the product topology. It is enough to show that $w^{*}=w$ : indeed, the group $\Gamma$ is completely determined by the shapes of a finite number of tetrahedra $\Delta_{i}$.

The main observation is that the words $\Omega^{n} \in\{R, L\}^{\mathbb{Z}}$ converge pointwise to $\Omega$, and $\phi^{ \pm, n} \rightarrow \phi^{ \pm}$(pointwise in $\mathbb{R}^{\mathbb{Z}}$ ), by definition (9). Therefore, $w^{*}$ belongs to the space $W$, hence the volume inequality $\mathcal{V}\left(w^{*}\right) \leq \mathcal{V}(w)$. Since $\max _{W} \mathcal{V}$ is achieved at a unique point (the volume is a strictly concave function), it is enough to prove the reverse inequality. We proceed by contradiction.

Suppose $\mathcal{V}\left(w^{*}\right)<\mathcal{V}(w)$. There exist integers $m<0<M$ such that the tetrahedra $\left\{\Delta_{i}\right\}_{m<i<M}$ defined by $w$ have total volume larger than $\mathcal{V}\left(w^{*}\right)$. If we can extend $\left(w_{i}\right)_{m \leq i \leq M}$ to a sequence $\left(v_{i}\right)_{i \in \mathbb{Z}}$ of $W^{n}$ for large enough $n$, we will obtain a contradiction.

By Corollary 5.2, we can assume $\frac{w_{m}}{\phi_{m}^{-}}>\frac{w_{m+1}}{\phi_{m+1}^{-}}$and $\frac{w_{M}}{\phi_{M}^{+}}>\frac{w_{M-1}}{\phi_{M-1}^{+}}$. Since $w$ satisfies (11) (especially the strong inequalities involving $\phi^{ \pm}$) for $n$ large enough, the restricted sequence $\left(w_{m}, \ldots, w_{M}\right)$ satisfies the corresponding inequalities defining $W^{n}$ by convergence of the $\phi^{ \pm, n}$. Pick such a large $n$ and define (redundantly at $i=m, M$ )

$$
v_{i}:= \begin{cases}\phi_{i}^{-, n} \frac{w_{m}}{\phi_{m}^{-, n}} & \text { if } i \leq m \\ w_{i} & \text { if } m \leq i \leq M \\ \phi_{i}^{+, n} \frac{w_{M}}{\phi_{M}^{+, n}} & \text { if } M \leq i\end{cases}
$$

If $n$ is large enough, it is easy to check that $\left(v_{i}\right)_{i \in \mathbb{Z}}$ belongs to $W^{n}$ : the only non-obvious thing is to make sure the triple $\left(v_{M-1}, v_{M}, v_{M+1}\right)$ (and similarly $\left(v_{m-1}, v_{m}, v_{m+1}\right)$ ) satisfies the last two conditions of (10). 
As $n$ becomes large, notice that the triple $\left(\frac{v_{M-1}}{\phi_{M-1}^{+}}, \frac{v_{M}}{\phi_{M}^{+}}, \frac{v_{M+1}}{\phi_{M+1}^{+}}\right)$goes to $\left(\frac{w_{M-1}}{\phi_{M-1}^{+}}, \frac{w_{M}}{\phi_{M}^{+}}, \frac{w_{M}}{\phi_{M}^{+}}\right)$, whose last two terms are equal to each other, and larger than the first one. The result then follows from Remark 3.3.

q.e.d.

Corollary 10.8 (C. Series). A quasifuchsian punctured-torus group $\Gamma$ is determined up to conjugacy in $\operatorname{Isom}\left(\mathbb{H}^{3}\right)$ by its pleating measures $\lambda^{ \pm}$.

Proof. In other words, the map $\Psi: \mathcal{L} \rightarrow \mathcal{Q F}$ is a homeomorphism. We already know that $\Psi$ is well-defined and injective (Theorems 1.1 and 10.1), and continuous (Proposition 10.7). Since $\mathcal{L}$ is a real manifold of dimension 4 , the theorem of domain invariance states that the image $\operatorname{Im}(\Psi)$ is open. It remains to show that $\operatorname{Im}(\Psi)$ is closed.

Consider pairs $\left(\lambda_{n}^{+}, \lambda_{n}^{-}\right)$such that the corresponding groups $\Gamma_{n}=$ $\Psi\left(\lambda_{n}^{+}, \lambda_{n}^{-}\right)$converge to some $\Gamma$ in $\mathcal{Q F}$. The function (here $\left.\Psi^{-1}\right)$ which to a group associates its pleatings is always continuous $[\mathbf{K S}]$, so the $\left(\lambda_{n}^{+}, \lambda_{n}^{-}\right)$converge to some $\left(\lambda^{+}, \lambda^{-}\right)$in $\mathcal{L}$. Proposition 10.7 then implies that $\Gamma=\Psi\left(\lambda^{+}, \lambda^{-}\right)$.

q.e.d.

Theorem 0.1 now follows from Theorem 10.1.

\section{References}

[Ak] H. Akiyoshi, On the Ford domains of once-punctured torus groups, in 'Hyperbolic spaces and related topics,' RIMS, Kyoto, Kokyuroku 1104 (1999) 109-121, MR 1744475, Zbl 0951.57505.

[AS] H. Akiyoshi \& M. Sakuma, Comparing two convex hull constructions of cusped hyperbolic manifolds, Proceedings of the Workshop 'Kleinian groups and hyperbolic 3-manifolds' (Warwick 2002), Lond. Math. Soc. Lecture Notes 299 (2003), 209-246, MR 2044552, Zbl 1048.57007.

[ASWY1] H. Akiyoshi, M. Sakuma, M. Wada, \& Y. Yamashita, Ford domains of punctured torus groups and two-bridge knot groups, in 'Knot Theory,' Proceedings of the workshop held in Toronto dedicated to 70th birthday of Prof. K. Murasugi, 1999, MR 1799369, Zbl 0969.57505.

[ASWY2] _ Jorgensen's picture of quasifuchsian punctured torus groups, Proceedings of the Workshop 'Kleinian groups and hyperbolic 3-manifolds' (Warwick 2002), Lond. Math. Soc. Lecture Notes 299 (2003) 247-273, MR 2044553.

[ASWY3] _ Punctured torus groups and 2-bridge knot groups (I), Lecture notes in Mathematics, 1909, Springer, 2007.

[B1] F. Bonahon, Bouts des variétés hyperboliques de dimension 3, Ann. of Math. 124 (1986) 71-158, MR 0847953, Zbl 0671.57008.

[B2] Shearing hyperbolic surfaces, bending pleated surfaces and Thurston's symplectic form, Ann. Fac. Sci. Toulouse Math. 5 (1996) 233297, MR 1413855, Zbl 0880.57005. 
[BO] F. Bonahon \& J.-P. Otal, Laminations mesurées de plissage des variétés hyperboliques de dimension 3, Ann. of Math. 160 (2004) 1013-1055, MR 2144972, Zbl 1083.57023.

[Bo] B.H. Bowditch, Markoff triples and quasifuchsian groups, Proc. Lond. Math. Soc. Vol. 77 (1998) 697-736, MR 1643429, Zbl 0928.11030.

[Br] M. Bridgeman, Average bending of convex pleated planes in hyperbolic three-space, Invent. Math. 132(3) (1998) 381-391, MR 1621436, Zbl 0912.30028.

[BCM] J. Brock, R. Canary, \& Y. Minsky, The classification of Kleinian surface groups II: the ending lamination conjecture, Arxiv: math.GT/0412006, 2004.

[CEG] R.D. Canary, D.B.A. Epstein, \& R. Green, Notes on notes of Thurston, in 'Analytical and Geometric Aspects of Hyperbolic Space' (Epstein ed.), Lond. Math. Soc. Lecture Notes, 111, Cambridge University Press, 1987, 3-92, MR 0903850, Zbl 0612.57009.

[CH] K. Chan, Constructing hyperbolic 3-manifolds, Undergraduate thesis with C. Hodgson, University of Melbourne, 2002.

[EP] D.B.A. Epstein \& R.C. Penner, Euclidean decompositions of noncompact hyperbolic manifolds, J. Differential Geom. 27 (1988) 67-80, MR 0918457, Zbl 0611.53036.

[FZ] S. Fomin \& A. Zelevinsky, The Laurent phenomenon, Adv. Applied Math. 28(2) (2002) 119-144, MR 1888840, Zbl 1012.05012.

[G1] F. Guéritaud, Formal Markoff maps are positive, Geometriae Dedicata 134 (2008), 203-216, MR 2399659.

[G2] Géométrie hyperbolique effective et triangulations idéales canoniques en dimension trois, Ph.D. thesis, Orsay, 2006.

[GF] - On canonical triangulations of once-punctured torus bundles and two-bridge link complements (with an Appendix by D. Futer), Geometry \& Topology 10 (2006) 1239-1284, MR 2255497.

[Jø] T. Jørgensen, On pairs of once-punctured tori, (unfinished manuscript), in 'Kleinian groups and hyperbolic 3-manifolds' (Y. Komori, V. Markovic, C. Series, Editors), Lond. Math. Soc. Lecture Notes 299 (2003) 183-207, MR 2044551, Zbl 1061.30038.

[KS] L. Keen \& C. Series, Continuity of convex hull boundaries, Pacific J. Math. 168(1) (1995) 183-206, MR 1331998, Zbl 0838.30043.

[La] M. Lackenby, The canonical decomposition of once-punctured torus bundles, Comment. Math. Helv. 78 (2003) 363-384, MR 1988201, Zbl 1035.57010.

[Mil] J. Milnor, Hyperbolic geometry: the first 150 years, Bull. Amer. Math. Soc. 6(1) (1982) 9-24, MR 0634431, Zbl 0486.01006.

[Min] Y. Minsky, The classification of punctured-torus groups, Annals of Math. 149 (1999) 559-626, MR 1689341, Zbl 0939.30034.

[MSW] D. Mumford, C. Series, \& D. Wright, Indra's Pearls, the Vision of Felix Klein, Cambridge Univ. Press, 2002, MR 1913879.

[Ot] J.-P. Otal, Le théorème d'hyperbolisation pour les variétés fibrées de dimension 3, Astérisque 235 (1996), MR 1402300, Zbl 0855.57003. 
[Pr] J. Propp, The combinatorics of frieze patterns and Markoff numbers, arXiv:math.CO/0511633, 2005.

[Ri] I. Rivin, Euclidean structures on simplicial surfaces and hyperbolic volume, Ann. of Math. 139 (1994) 553-580, MR 1283870, Zbl 0823.52009.

[Se] C. Series, Thurston's bending measure conjecture for once punctured torus groups, Lond. Math. Soc. Lecture Notes, 329, Cambridge Univ. Press, 2006, MR 2258745.

[Th] W.P. Thurston, Hyperbolic Structures on 3-manifolds, II: Surface groups and 3-manifolds which fiber over the circle, Arxiv: math.GT/9801045, 1998.

[Wa] M. Wada, Opti, a computer program for the study of punctured-torus groups: http://vivaldi.ics.nara-wu.ac.jp/ ${ }^{\text {wada/OPTi/. }}$

Université DE LILle I, BÂT. M2-M3 Laboratoire de mathématiques Paul Painlevé

U.M.R. CNRS 8524

59655 Villeneuve d'Ascq Cedex, France E-mail address: Francois.Gueritaud@normalesup.org 